\title{
Derived functors of nonadditive functors and homotopy theory
}

\author{
LAWRENCE BREEN \\ ROMAN MIKHAILOV
}

\begin{abstract}
The main purpose of this paper is to extend our knowledge of the derived functors of certain basic nonadditive functors. The discussion takes place over the integers, and includes a functorial description of the derived functors of certain Lie functors, as well as that of the main cubical functors. We also present a functorial approach to the study of the homotopy groups of spheres and of Moore spaces $M(A, n)$, based on the Curtis spectral sequence and the decomposition of Lie functors as iterates of simpler functors such as the symmetric or exterior algebra functors. As an illustration, we retrieve in a purely algebraic manner the 3-torsion components of the homotopy groups of the 2-sphere in low degrees, and give a unified presentation of the homotopy groups $\pi_{i}(M(A, n))$ for small values of both $i$ and $n$.
\end{abstract}

18G55, 18G10; 54E30, 55Q40

\section{Introduction}

A great number of methods for computing the homotopy or the homology of a topological space begin with a mod $p$ reduction, and this has proved to be very efficient even though one then has to deal with an extension problem when reverting to integer coefficients. However, such methods are not well-suited when one considers spaces which are of an algebraic nature, such a Eilenberg-Mac Lane spaces. That a purely functorial approach is possible in such a case was already apparent in the classical paper of S Eilenberg and S Mac Lane [22], in which they explicitly compute the integral homology of Eilenberg-Mac Lane spaces in low degrees. Their results are expressed in terms of what they called the "new and quite bizarre functors" $\Omega(\Pi)$ and $R(\Pi)$. These functors became more intelligible with the advent of the Dold-Puppe theory of derived functors of nonadditive functors [20], as they could then be interpreted as the leftderived functors of the second exterior power functor and of the second divided power functor respectively. Higher analogues of these new functors subsequently appeared in related contexts in a number a places, particularly in the $\mathrm{PhD}$ theses of Mac Lane's students, R Hamsher [28] and G Decker [17]. However, this line of research was not 
vigorously pursued, though one should mention in this context the works of $\mathrm{H}$ Baues [2] and of the first author [10], as well as the unpublished preprint of A K Bousfield [8].

In the present text, we compute in this functorial spirit certain unstable homotopy groups of Moore spaces $M(A, n)$ and in particular of the corresponding spheres $S^{n}=M(\mathbb{Z}, n)$. This approach to the computation of the homotopy groups of spheres is of particular interest, since much more structure is revealed when these homotopy groups are described as special values at the group $A=\mathbb{Z}$ of appropriate functors. Our method is in some sense quite classical, since it relies on D Kan's construction of the loop group $G K$ of a connected simplicial set $K$ and on E Curtis' spectral sequence determined by the lower central series filtration of $G K$. The initial terms in this spectral sequence were described by Curtis in [15] in terms of the derived functors of the Lie functors $\mathscr{L}^{n}$. He showed in addition that these Lie functors are endowed with a natural filtration, whose associated graded components are built up from more familiar functors.

It follows from this description that a key ingredient in such an approach must be a good understanding of the derived functors of the functor $\mathscr{L}^{n}$. We are able to achieve this in low degrees, where this is made possible by the fact that this Curtis decomposition of the Lie functors reduces this problem to the computation of derived functors of iterates of certain elementary functors (particularly the degree $r$ symmetric functor $\mathrm{SP}^{r}$, and the related $r$-th exterior algebra and $r$-th divided power functors $\Lambda^{r}$ and $\Gamma_{r}$ ). In order to deal with such iterates, we require a composite functor spectral sequence along the lines of the standard Grothendieck composite functor spectral sequence described for example in Weibel [47], but now for a pair of composable nonadditive functors. Such a nonadditive composite functor spectral sequence has been discussed by D Blanc and C Stover [6], but we give here a formulation of its initial terms which is better suited to our computational objectives. In fact this spectral sequence degenerates in our context at $E^{2}$, and may rather be thought of, for the functors which we consider, as a symmetrization of the Künneth formula and its higher analogues à la Mac Lane $[34 ; 35]$. It allows us to go beyond the computation of the derived functors of iterates of $\Lambda^{2}$ considered in this context by the second author and IB Passi in [40].

In our quest for the explicit values of the Dold-Puppe derived functors of the Lie algebra functors $\mathscr{L}^{r}$ for certain values of $r$, we deal with a number of questions of independent interest. First of all, starting from the description of the derived functors of $\mathrm{SP}^{r}$ and $\Lambda^{r}$ given by F Jean (a student of the first author) in his thesis [30], we obtain a complete description of these derived functors (as well as those of the divided power functor $\Gamma_{r}$ ) for $r=2$, by a method different from that of $\mathrm{H}$ Baues and T Pirashvili in [5]. We then go on to give a similarly complete and functorial description of the corresponding derived functors of $\mathrm{SP}^{3}, \Lambda^{3}$ and $\Gamma_{3}$, and we deduce from this a functorial description 
of the derived functors $L_{i} \mathscr{L}^{3}(A, n)$ for all $i$ and $n$. We also compute certain derived functors of the quartic composite functors $\Lambda^{2} \Lambda^{2}$ and $\Lambda^{2} \Gamma_{2}$, and deduce from these certain values of the derived functors $L_{i} \mathscr{L}^{4}(A, n)$.

We were led, in our quest for the derived functors of $\mathscr{L}^{4}$, to an analogue for Lie functors of the décalage morphisms. The latter are maps determined by the existence of Koszul complexes, and which relate to each other the derived functors of $\mathrm{SP}^{r}, \Lambda^{r}$ and $\Gamma_{r}$. Just as $\Lambda^{r}$ may be viewed as a superanalogue ${ }^{1}$ of $\mathrm{SP}^{r}$, we need to introduce a superanalogue $\mathscr{L}_{s}^{r}$ of the Lie functor $\mathscr{L}^{r}$. It turns out that this must not be the naive graded commutative version of the Lie functor, in which certain signs are changed in the relations defining it. Instead, it is necessary to introduce in its definition, following D Leibowitz in her unpublished thesis [33], an additional divided square operation with respect to the Lie superbracket of odd degree elements. While there no longer exist décalage isomorphisms between the Lie and super-Lie functors, there do exist (in Bousfield's terminology) canonical pension maps between them, which we call the semi-décalage morphisms. These give us a refined description of the derived functors of certain super-Lie functors.

We rely at some point on the knowledge of the homology of the complex $C^{n}(A)$ associated to an abelian group $A$. This is the dual of the de Rham complex first introduced in the present context by V Franjou, J Lannes and L Schwartz in [24]. We refer to Mikhailov [39] for an explicit calculation of the values of the homology groups $H_{0} C^{n}(A)$ for all $n$, as announced by Jean in [30], as well as for a description of all the homology groups $H_{i} C^{n}(A)$ when $n<8$. The occurrence of 4-torsion in $H_{1} C^{8}(A)$ and that of a Lie functor term in $H_{1} C^{6}(A)$, suggests that there is no simple functorial description of these groups for a general $n$.

In Section 11, we use these tools in order to achieve our goal of computing algebraically certain homotopy groups of $n$-spheres and Moore spaces $M(A, n)$. The task at hand is twofold. First of all, as we have said, one must compute the initial terms of the Curtis spectral sequence, and for this we rely on our knowledge of the derived functors of certain Lie functors and their superanalogues. The second part consists in understanding certain differentials in the spectral sequence. We rely here upon various methods, some based on the functoriality of our construction and on the fact that the differentials are therefore natural transformations rather then mere group homomorphisms. Others are more classical in spirit (the suspension of a Moore space, the comparison of a Moore space with the corresponding Eilenberg-Mac Lane space, and so forth). We have at times in this final section made use of known results concerning the homotopy of

\footnotetext{
${ }^{1}$ We prefer to call this the superanalogue, rather than the graded analogue as is more customary, since all our functors are graded.
} 
Moore spaces for specific groups $A$, whenever this allowed us to progress with our own investigations. It is quite striking to observe how far one can go in the description of these homotopy groups, with only the knowledge of derived functors of quadratic and cubical functors as the basic input.

In this final section we proceed in logical order, beginning with the homotopy groups of $S^{2}$ and $M(A, 2)$ and then moving on to $M(A, n)$ for increasing values of $n$. For $A=\mathbb{Z}$, some of our results follow in the stable range from the six author paper Bousfield et al [9] where a more efficient spectral sequence is considered, which however depends on the choice of a fixed prime $p$ (see also Curtis [16]). As an illustration of our methods, we begin by explaining how one may obtain in this way the known values of the 3-torsion of $\pi_{i}\left(S^{2}\right)$, for values of $i$ up to 14 . To have gone further, so as to retrieve the classical results of $\mathrm{H}$ Toda [46] up to $i=22$, would have obliged us to delve further into the analysis of the spectral sequence. We also recover, and reinterpret, some results of Baues and his collaborators $[2 ; 21 ; 3]$. In particular, we obtain by our methods the results of Baues and Buth [3] concerning $\pi_{i}(M(A, 2))$ for $i=4$, and give an improved description of the unpublished results of Dreckmann for $i=5$ [21] (see also Baues and Goerss [4], and the recent Mikhailov and $\mathrm{Wu}$ [41]). Specializing to the case $A=\mathbb{Z} / p$, we obtain further information about the groups $\pi_{i}(M(\mathbb{Z} / p, 2))$ whenever the prime $p$ is odd. By suspending these calculations, this gives us in particular a fully functorial description of the graded components associated to a natural filtration of the group $\pi_{6}(M(A, 3))$ which appears to be new. As a consequence of these computations, we can recover the value of $\pi_{5}(M(\mathbb{Z} / 3,2))$, a significant case of the extension by Neisendorfer [42] to the prime $p=3$ of Cohen, Moore and Neisendorfer's study [12] of the homotopy of Moore spaces (this value had in fact already been obtained by Leibowitz in [33]).

As a final example, we examine the low degree homotopy groups of $M(\mathbb{Z} / 3,5)$ since this allows us to exhibit in a simple context some of the techniques on which we relied throughout this section. In fact, the reader may wish to begin with this case, before going on to the more delicate unstable computations which precede it.

As will be apparent from this description of our paper, a number of our results in homotopy theory have already appeared in one form or another in the literature, where they are proved by very diverse methods. Our aim here is to show that these can all be obtained by a uniform method, based solely on functorial techniques from homological and homotopical algebra with integer coefficients. We expect that such an approach to these questions will not only allow one to compute specific additional homotopy groups, but more importantly will shed some new light upon their global structure. 
Acknowledgements We are indebted to P Goerss for providing us with a copy of the dissertation of Dreckmann [21], and to A K Bousfield for making available to us the thesis of Leibowitz [33] as well as for his comments regarding a first version of the present text. The research of the second author is partially supported by RFBR Grant 10-01-93113-NCNIL ${ }_{a}$, Dynasty grant and RF Presidential grant MK-3644.2009.01.

\section{Derived functors}

\subsection{Graded functors}

Let $\mathrm{Ab}$ be the category of abelian groups and $A$ an object of $A b$. For any chain complex $C_{i}$, we will henceforth denote by $C[n]$ the chain complex defined by

$$
C[n]_{i}:=C_{i-n} \text { for all } i .
$$

In particular, the chain complex $A[n]$ is concentrated in degree $n$. In addition to the graded tensor power functor $\otimes:=\bigoplus_{n \geq 0} \otimes^{n}$, the symmetric power functor SP $:=$ $\bigoplus_{n \geq 0} \mathrm{SP}^{n}$, and the exterior power functor $\Lambda:=\bigoplus_{n \geq 0} \Lambda^{n}$ (the quotient of $\otimes A$ by the ideal generated by elements $x \otimes x$ for all $x \in A$ ), we will consider over $\mathbb{Z}$ the following somewhat less well-known functors:

Divided power functors (See Roby [44].) $\Gamma_{*}=\bigoplus_{n \geq 0} \Gamma_{n}: \mathrm{Ab} \rightarrow \mathrm{Ab}$. The graded algebra $\Gamma_{*}(A)$ is generated by symbols $\gamma_{i}(x)$ of degree $i \geq 0$ satisfying the following relations for all $x, y \in A$ :

(1) $\gamma_{0}(x)=1$

(2) $\gamma_{1}(x)=x$

(3) $\gamma_{s}(x) \gamma_{t}(x)=\left(\begin{array}{c}s+t \\ s\end{array}\right) \gamma_{s+t}(x)$

(4) $\gamma_{n}(x+y)=\sum_{s+t=n} \gamma_{s}(x) \gamma_{t}(y), n \geq 1$

(5) $\gamma_{n}(-x)=(-1)^{n} \gamma_{n}(x), n \geq 1$.

In particular, the canonical map $A \simeq \Gamma_{1}(A)$ is an isomorphism. The degree 2 component $\Gamma_{2}(A)$ of $\Gamma_{*}(A)$ is the Whitehead functor $\Gamma(A)$. It is universal for homogenous quadratic maps from $A$ into abelian groups. The following additional relations in $\Gamma_{*}(A)$ 
are consequences of the previous ones:

$$
\begin{aligned}
\gamma_{r}(n x) & =n^{r} \gamma_{r}(x), \quad n \in \mathbb{Z} \\
r \gamma_{r}(x) & =x \gamma_{r-1}(x) \\
x^{r} & =r ! \gamma_{r}(x) \\
\gamma_{r}(x) y^{r} & =x^{r} \gamma_{r}(y) .
\end{aligned}
$$

In addition, a direct computations implies that

$$
\Gamma_{r}(\mathbb{Z} / n) \simeq \mathbb{Z} / n\left(r, n^{\infty}\right),
$$

where the extended $\operatorname{gcd}\left(r, n^{\infty}\right)$ is defined by $\left(r, n^{\infty}\right):=\lim _{m \rightarrow \infty}\left(r, n^{m}\right)$.

Lie functors $\mathscr{L}: \mathrm{Ab} \rightarrow \mathrm{Ab} \quad$ (See Curtis [14].) The tensor algebra $\otimes(A):=\bigoplus_{n}\left(\otimes^{n} A\right)$ on a $\mathbb{Z}$-module $A$ is endowed with a $\mathbb{Z}$-Lie algebra structure, for which the bracket operation is defined by

$$
[a, b]:=a \otimes b-b \otimes a, \quad a, b \in \otimes(A) .
$$

One defines $n$-fold brackets inductively by setting

$$
\left[a_{1}, \ldots, a_{n}\right]:=\left[\left[a_{1}, \ldots, a_{n-1}\right], a_{n}\right] .
$$

We will denote $\otimes A$, viewed as a $\mathbb{Z}$-Lie algebra, by $\otimes(A)^{\text {Lie }}$. Let $\mathscr{L}(A)=\bigoplus_{n \geq 1} \mathscr{L}^{n}(A)$ be the sub-Lie ring of $\otimes(A)^{\text {Lie }}$ generated by $A$. Its degree 2 and 3 components are generated by the expressions

$$
a \otimes b-b \otimes a \quad \text { and } \quad a \otimes b \otimes c-b \otimes a \otimes c+c \otimes a \otimes b-c \otimes b \otimes a
$$

where $a, b, c \in A . \mathscr{L}(A)$ is called the free Lie ring generated by the abelian group $A$. It is universal for homomorphisms from $A$ to $\mathbb{Z}$-Lie algebras. The grading of $\otimes(A)$ determines a grading on $\mathscr{L}(A)$, so that we obtain a family of endofunctors on the category of abelian groups:

$$
\mathscr{L}^{i}: \mathrm{Ab} \rightarrow \mathrm{Ab}, \quad i \geq 1 .
$$

In particular,

$$
\mathscr{L}^{i}(\mathbb{Z})=0
$$

for all $i>1$. For any free group $F$ and $i \geq 1$, one has the natural Magnus-Witt isomorphism (see Magnus [36] and Witt [49])

$$
\gamma_{i}(F) / \gamma_{i+1}(F) \simeq \mathscr{L}^{i}\left(F_{\mathrm{ab}}\right),
$$

where $\gamma_{i}(F)$ is the $i$-th term in the lower central series of $F$. 
Schur functors We will also consider the Schur functors

$$
J^{n}, Y^{n}, E^{n}: \mathrm{Ab} \rightarrow \mathrm{Ab}, \quad n \geq 2
$$

defined by

$$
\begin{aligned}
& J^{n}(A)=\operatorname{ker}\left\{A \otimes \operatorname{SP}^{n-1}(A) \rightarrow \operatorname{SP}^{n}(A)\right\}, \quad n \geq 2, \\
& Y^{n}(A)=\operatorname{ker}\left\{A \otimes \Lambda^{n-1}(A) \rightarrow \Lambda^{n}(A)\right\}, \quad n \geq 2, \\
& E^{n}(A)=\operatorname{ker}\left\{A \otimes \Gamma_{n-1}(A) \rightarrow \Gamma_{n}(A)\right\}, \quad n \geq 2 \text {. }
\end{aligned}
$$

In particular,

$$
J^{2}(A)=E^{2}(A) \simeq \Lambda^{2} A \text { and } \quad Y^{2}(A) \simeq \Gamma_{2}(A)
$$

whenever $A$ is free. The functors $Y^{n}(A)$ are the $\mathbb{Z}$-forms of the Schur functors $\mathbb{S}_{\lambda}(V)$ associated to the partition $\lambda=(2,1 \ldots, 1)$ of the set $(n)$ (see Fulton and Harris [26, Exercise 6.11] and Fulton [25, Chapter 8 (19)]). The functors $J^{n}(A)$ and $E^{n}(A)$ are two distinct $\mathbb{Z}$-forms of the Schur functors $\mathbb{S}_{\mu}$ associated to the partition $\mu=(n-1,1)$ of $(n)$, which is the conjugate of the partition $\lambda$.

The functors $J^{n}$ and their derived functors were considered by E Curtis [15] and J Schlesinger [45]. Just as the quotient of a Lie ring $\mathscr{L}$ by the ideal generated by all brackets is an abelian Lie ring, one can consider the metabelian Lie rings. These are the quotients of $\mathscr{L}$ by the ideal generated by brackets of the form [[ ], [ ]]. The following proposition asserts that the functors $J^{n}$, restricted to free abelian groups $A$, are metabelian Lie functors:

Proposition 2.1 (Schlesinger) [45] Let $A$ be a free abelian group and $n \geq 2$. The 4-term sequence

$$
0 \rightarrow \mathscr{L}^{n}(A) \cap \mathscr{L}^{2} \mathscr{L}^{2}(A) \rightarrow \mathscr{L}^{n}(A) \stackrel{p_{n}}{\longrightarrow} A \otimes \operatorname{SP}^{n-1}(A) \stackrel{r_{n}}{\longrightarrow} \operatorname{SP}^{n}(A) \rightarrow 0
$$

is exact, where $r_{n}$ is the multiplication, and the map $p_{n}$ is defined by

$$
p_{n}:\left[m_{1}, \ldots, m_{n}\right] \mapsto m_{1} \otimes m_{2} \cdots m_{n}-m_{2} \otimes m_{1} m_{3} \cdots m_{n} \text {, where } m_{j} \in A \forall j .
$$

The projection

$$
\mathscr{L}^{n}(A) \longrightarrow J^{n}(A)
$$

of $\mathscr{L}^{n}(A)$ onto its image in $A \otimes \mathrm{SP}^{n-1} A$ will also be denoted $p_{n}$, so that if we set

$$
\widetilde{J}^{n}(A):=\mathscr{L}^{n}(A) \cap \mathscr{L}^{2} \mathscr{L}^{2}(A),
$$


the sequence (2-7) splits into a pair of short exact sequences:

$$
\begin{gathered}
0 \longrightarrow \tilde{J}^{n}(A) \longrightarrow \mathscr{L}^{n}(A) \stackrel{p_{n}}{\longrightarrow} J^{n}(A) \longrightarrow 0 \\
0 \longrightarrow J^{n}(A) \longrightarrow \mathrm{SP}^{n-1} \longrightarrow \mathrm{SP}^{n}(A) \longrightarrow 0 .
\end{gathered}
$$

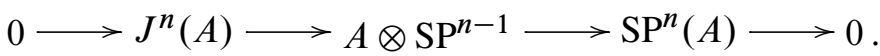

In particular,

$$
\mathscr{L}^{2}(A) \simeq \Lambda^{2}(A) \text { and } \quad \mathscr{L}^{3}(A) \simeq J^{3}(A)
$$

since $\widetilde{J}^{n}(A)$ is trivial for $n=2,3$.

There is a natural transformation

$$
\mathbb{Z}[A] \longrightarrow \Gamma_{n}(A)
$$

which sends $[a]$ to $\gamma_{n}(a)$. The algebra structure on $\Gamma_{*}(A)$ determines for each $n$ a natural transformation

$$
\mathrm{SP}^{n}(A) \longrightarrow \Gamma_{n}(A) .
$$

In addition, there are natural transformations:

$$
\begin{aligned}
f_{n}: \operatorname{SP}^{n}(A) & \rightarrow \otimes^{n} A \\
a_{1} \cdots a_{n} & \mapsto \sum_{\sigma \in \Sigma_{n}} a_{i_{1}} \otimes \cdots \otimes a_{i_{n}} \\
g_{n}: \Lambda^{n}(A) & \rightarrow \otimes^{n} A \\
a_{1} \wedge \cdots \wedge a_{n} & \mapsto \sum_{\sigma \in \Sigma_{n}} \operatorname{sign}(\sigma) a_{i_{1}} \otimes \cdots \otimes a_{i_{n}} \\
h_{n}: \Gamma_{n}(A) & \rightarrow \otimes^{n} A \\
\gamma_{r_{1}}\left(a_{1}\right) \cdots \gamma_{r_{k}}\left(a_{k}\right) & \mapsto \sum_{\left(i_{1}, \ldots, i_{n}\right)} a_{i_{1}} \otimes \cdots \otimes a_{i_{n}}
\end{aligned}
$$

In these definitions of $f_{n}$ and $g_{n}$ we have set $i_{j}:=\sigma(j)$, whereas the $\left(i_{1}, \ldots, i_{n}\right)$ in the definition of $h_{n}$ range over the set of $n$-tuples of integers for which $j$ occurs $r_{j}$ times $(1 \leq j \leq k)$. When $A$ is free abelian, the induced morphism

$$
h_{n}: \Gamma_{n}(A) \rightarrow\left(\otimes^{n} A\right)^{\Sigma_{n}}
$$

from $\Gamma_{n}(A)$ to the group of tensors invariant under the action of the symmetric group is an isomorphism for all $n \geq 1$. By the universal property of the algebra $\Gamma_{*}(A), h_{n}$ may 
also be characterized as the map determined by the divided power algebra structure on $\otimes(A)$, where the product in this algebra is defined by the shuffle product, and the divided powers are characterized by the rule $\gamma_{n}(a):=a \otimes \cdots \otimes a \in \otimes^{n} A$ for all $a \in A$.

\subsection{Derived functors}

Let $A$ be an abelian group, and $F$ an endofunctor on the category of abelian groups. Recall that for every $n \geq 0$ the derived functor of $F$ in the sense of A Dold and D Puppe [20] are defined by

$$
L_{i} F(A, n)=\pi_{i}\left(F K P_{*}[n]\right), \quad i \geq 0
$$

where $P_{*} \rightarrow A$ is a projective resolution of $A$, and $K$ is the Dold-Kan transform, inverse to the Moore normalization functor

$$
N: \operatorname{Simpl}(\mathrm{Ab}) \rightarrow C(\mathrm{Ab})
$$

from simplicial abelian groups to chain complexes (see for example Weibel [47, Definition 8.3.6]). We denote by $L F(A, n)$ the object $F K\left(P_{*}[n]\right)$ in the homotopy category of simplicial abelian groups determined by $F K\left(P_{*}[n]\right)$, so that

$$
L_{i} F(A, n)=\pi_{i}(L F(A, n)) .
$$

We set $L F(A):=L F(A, 0)$ and $L_{i} F(A):=L_{i} F(A, 0)$ for all $i \geq 0$. When the functor $F$ is additive, the $L_{i} F(A)$ are isomorphic by iterated suspension to $L_{i+n} F(A, n)$ for all $n$, and coincide with the usual derived functors of $F$. As examples of these constructions, note that the simplicial models $L F(L \rightarrow M)$ of $L F A$ and $F K((L \rightarrow M)[1])$ of $L F(A, 1)$ associated to the two-term flat resolution

$$
0 \rightarrow L \stackrel{f}{\rightarrow} M \rightarrow A \rightarrow 0
$$

of an abelian group $A$ are respectively of the following form in low degrees:

$$
F\left(s_{0}(L) \oplus s_{1}(L) \oplus s_{1} s_{0}(M)\right) \stackrel{\partial_{0}, \partial_{1}, \partial_{2}}{\rightleftarrows} F\left(L \oplus s_{0}(M)\right) \stackrel{\stackrel{\partial_{0}, \partial_{1}}{\rightleftarrows}}{\rightleftarrows} F(M)
$$

where the component $F(M)$ is in degree zero, and

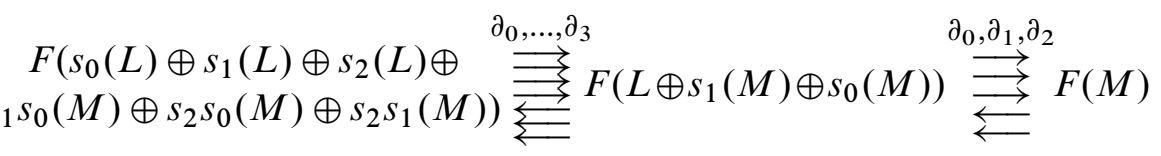

where the component $F(M)$ is in degree 1. It follows from the definition of homology that $L_{i} \mathbb{Z}(A, n) \simeq H_{i}(K(A, n) ; \mathbb{Z})$ for all $n$, where $K(A, n)$ is an Eilenberg-MacLane space associated to the abelian group $A$. 
Derived functors of $\otimes^{n}$ (See Mac Lane [35].) For $n \geq 1$, and abelian groups $A_{1}, \ldots, A_{n}$, we define ${ }^{2}$

$$
\operatorname{Tor}_{i}\left(A_{1}, \ldots, A_{n}\right):=H_{i}\left(A_{1} \stackrel{L}{\otimes} \cdots \stackrel{L}{\otimes} A_{n}\right), \quad i \geq 0
$$

where $A \stackrel{L}{\otimes} B$ is the derived tensor product of the abelian groups $A$ and $B$ in the derived category of abelian groups, as in Weibel [47, Section 10.6]. In particular,

$$
\operatorname{Tor}_{0}\left(A_{1}, \ldots, A_{n}\right) \simeq A_{1} \otimes \cdots \otimes A_{n} \quad \text { and } \quad \operatorname{Tor}_{i}\left(A_{1}, \ldots, A_{n}\right)=0, \quad i \geq n .
$$

One sets

$\operatorname{Tor}\left(A_{1}, A_{2}\right):=\operatorname{Tor}_{1}\left(A_{1}, A_{2}\right)$ and $\operatorname{Tor}^{[n]}(A):=\operatorname{Tor}_{n-1}(A, \ldots, A)(n$ copies of $A)$.

While computations of such iterated Tor functors for specific abelian groups $A$ are elementary, an explicit functorial description of the multifunctors Tor $_{i}$ is more delicate. The Künneth sequence

$$
0 \rightarrow \operatorname{Tor}\left(A_{1}, A_{2}\right) \otimes A_{3} \rightarrow \operatorname{Tor}_{1}\left(A_{1}, A_{2}, A_{3}\right) \rightarrow \operatorname{Tor}\left(A_{1} \otimes A_{2}, A_{3}\right) \rightarrow 0,
$$

is functorial and exact but only splits unnaturally, as discussed in Mac Lane [34; 35]. The Eilenberg-Zilber theorem determines natural isomorphisms

$$
L_{i} \otimes^{n} A \simeq \operatorname{Tor}_{i}(A, \ldots, A) \text { for all } i \geq 0 .
$$

The group $\operatorname{Tor}^{[n]}(A)$ is generated by $n$-linear expressions $\tau_{h}\left(a_{1}, \ldots, a_{n}\right)$. Here the $a_{i}$ live in the subgroup ${ }_{h} A$ of elements $a$ of $A$ for which $h a=0(h>0)$, subject to the so-called slide relations

$$
\tau_{h k}\left(a_{1}, \ldots, a_{i}, \ldots, a_{n}\right)=\tau_{h}\left(k a_{1}, \ldots, k a_{i-1}, a_{i}, k_{i+1}, \ldots, k a_{n}\right)
$$

for all $i$, where $h k a_{j}=0$ for all $j \neq i$ and $h a_{i}=0$. The associativity of the derived tensor product functor implies that there are canonical isomorphisms

$$
\operatorname{Tor}^{[n]}(A) \simeq \operatorname{Tor}\left(\operatorname{Tor}^{[n-1]}(A), A\right), \quad n \geq 2 .
$$

The description of derived functors $L_{i} \otimes^{n} A$ for a general $i$ follows from that of $\operatorname{Tor}^{[n]}(A)$. For every abelian group $A, n \geq 1,1 \leq i \leq n-1$, the group $L_{i} \otimes^{n}(A)$ is by [35] the quotient

$$
L_{i} \otimes^{n}(A) \simeq \operatorname{Tor}^{[i+1]}(A) \otimes\left(\otimes^{n-i-1}(A)\right) / \mathrm{Jac}_{\otimes},
$$

${ }^{2}$ In [34], Mac Lane uses the notation $\operatorname{Trip}\left(A_{1}, A_{2}, A_{3}\right)$ for the group $\operatorname{Tor}_{1}\left(A_{1}, A_{2}, A_{3}\right)$ and $\operatorname{Tor}\left(A_{1}, \ldots, A_{n}\right)$ for $\operatorname{Tor}_{n-1}\left(A_{1}, \ldots, A_{n}\right)$. 
where $\mathrm{Jac}_{\otimes}$ is the subgroup of generalized Jacobi-type relations, generated by the expressions

$$
\sum_{k=1}^{i+2}(-1)^{k} \tau_{h}\left(x_{1}, \ldots, \widehat{x_{k}}, \ldots, x_{i+2}\right) \otimes x_{k} \otimes x_{i+3} \otimes \cdots \otimes x_{n}
$$

for all $x_{1}, \ldots, x_{n} \in A$.

Derived functors of $\mathrm{SP}^{n}$ The natural transformation $\otimes^{n} \longrightarrow \mathrm{SP}^{n}$ induces a natural epimorphism

$$
\operatorname{Tor}^{[n]}(A) \rightarrow L_{n-1} \mathrm{SP}^{n}(A)
$$

sending the generators $\tau_{h}\left(a_{1}, \ldots, a_{n}\right)$ of $\operatorname{Tor}^{[n]}(A)$ to generators $\beta_{h}\left(a_{1}, \ldots, a_{n}\right)$ of

$$
\mathcal{S}_{n}(A):=L_{n-1} \operatorname{SP}^{n}(A) .
$$

The kernel of this map is generated by the elements $\tau_{h}\left(a_{1}, \ldots, a_{n}\right)$ with $a_{i}=a_{j}$ for some $i \neq j$. It is shown by Jean in [30] that

$$
L_{i} \operatorname{SP}^{n}(A) \simeq\left(L_{i} \operatorname{SP}^{i+1}(A) \otimes \mathrm{SP}^{n-(i+1)}(A)\right) / \mathrm{Jac}_{\mathrm{SP}}
$$

where $\mathrm{Jac}_{\mathrm{SP}}$ is the subgroup generated by elements of the form

$$
\sum_{k=1}^{i+2}(-1)^{k} \beta_{h}\left(x_{1}, \ldots, \hat{x}_{k}, \ldots, x_{i+2}\right) \otimes x_{k} y_{1} \cdots y_{n-i-2}
$$

with $x_{i} \in{ }_{h} A$ and $y_{j} \in A$ for all $i, j$. The filtration of $\mathbb{Z}(A, n)$ by powers of the augmentation ideal determine a filtration on the homology groups $H_{r}(K(A, n) ; \mathbb{Z})$, whose associated graded pieces are the $L_{r} \operatorname{SP}^{s}(A, n)$ (see Breen [10]).

Derived functors of $\Lambda^{n}$ For any abelian group $A$ and $n \geq 1$, we set

$$
\Omega_{n}(A):=L_{n-1} \Lambda^{n}(A) .
$$

Consider the action of the symmetric group $\Sigma_{n}$ on $\operatorname{Tor}^{[n]}(A)$, defined by

$$
\sigma \tau_{h}\left(a_{1}, \ldots, a_{n}\right)=\operatorname{sign}(\sigma) \tau_{h}\left(a_{\sigma(1)}, \ldots, a_{\sigma(n)}\right)
$$

where $h a_{1}=\cdots=h a_{n}=0, a_{i} \in A, \sigma \in \Sigma_{n}$. We denote this action by $\Sigma_{n}^{\epsilon}$. The natural transformations $g_{n}$ induces functorial isomorphisms between $\Omega_{n}(A)$ and the $\Sigma_{n}^{\epsilon}$-invariants in $\operatorname{Tor}^{[n]}(A)[10 ; 30$, Theorem 2.3.3]:

$$
\Omega_{n}(A) \simeq\left(\operatorname{Tor}^{[n]}(A)\right)^{\Sigma_{n}^{\epsilon}} .
$$


In particular,

$$
\Omega_{n}(\mathbb{Z} / r) \simeq \mathbb{Z} / r .
$$

for all $n>0$ and $\Omega_{2}(A)$ is the functor $\Omega(A)$ of Eilenberg-Mac Lane [22]. The morphisms $\tau_{h}$ which described the Tor functors now symmetrize to homomorphisms

$$
\lambda_{h}^{n}: \Gamma_{n}\left({ }_{h} A\right) \rightarrow \Omega_{n}(A)
$$

for $h \geq 1$ and the group $\Omega_{n}(A)$ is generated by the elements

$$
\omega_{i_{1}}^{h}\left(x_{1}\right) * \cdots * \omega_{i_{j}}^{h}\left(x_{j}\right):=\lambda_{h}^{n}\left(\gamma_{i_{1}}\left(x_{1}\right) \cdots \gamma_{i_{j}}\left(x_{j}\right)\right)
$$

with $i_{k} \geq 1$ for all $k$, and $\sum_{k} i_{k}=n$. These satisfy relations which may be thought of, as in [10], as symmetrized versions of the slide relations (2-18). The following description of the derived functors $L_{i} \Lambda^{n}(A)$ is given in [30, Theorem 2.3.5]:

$$
L_{i} \Lambda^{n}(A) \simeq\left(\Omega_{i+1}(A) \otimes \Lambda^{n-i-1}(A)\right) / \mathrm{Jac}_{\Lambda} .
$$

Here $\mathrm{Jac}_{\Lambda}$ is the subgroup generated by the expressions

$$
\sum_{k=1}^{j} \omega_{i_{1}}^{h}\left(x_{1}\right) * \cdots * \omega_{i_{k}-1}^{h}\left(x_{k}\right) * \cdots * \omega_{i_{j}}^{h}\left(x_{j}\right) \otimes x_{k} \wedge y_{1} \wedge \cdots y_{n-i-2}
$$

for all $h$, with $\sum_{k=1}^{j}=i+2$. In particular, this implies that for any finite cyclic group $A$

$$
L_{i} \Lambda^{n}(A)=0 \text { for } i \neq n-1 .
$$

Derived functors of $\Gamma_{n}$ Not all is known about derived functors of the divided power functors. For an abelian group $A$, the double décalage isomorphism (described in (2-41) below) determines a composite isomorphism

$$
L_{1} \Gamma_{2}(A) \simeq L_{5} \operatorname{SP}^{2}(A, 2) \simeq H_{5}(K(A, 2), \mathbb{Z})
$$

so that $L_{1} \Gamma_{2}(A)$ is isomorphic to the functor $R(A)$ of Eilenberg-Mac Lane [22, Section 22], defined as

$$
\left(\operatorname{Tor}(A, A) \oplus \Gamma_{2}\left({ }_{2} A\right)\right) / S,
$$

where $S$ is the subgroup generated by elements

$$
\begin{array}{ll}
\tau_{h}(x, x), & x \in{ }_{h} A, h \in \mathbb{N}, \\
\gamma_{2}(x+y)-\gamma_{2}(x)-\gamma_{2}(y)-\tau_{2}(x, y), & x, y \in{ }_{2} A .
\end{array}
$$

More generally, we set

$$
R_{n}(A):=L_{n-1} \Gamma_{n}(A)
$$


so that $R_{2}(A)=R(A)$, even though this is inconsistent with the notation in Decker [17]. The sequence

$$
0 \longrightarrow \mathrm{SP}^{2}(A) \longrightarrow \Gamma_{2}(A) \longrightarrow A \otimes \mathbb{Z} / 2 \longrightarrow 0
$$

is exact for any abelian group $A$, and derives to the short exact sequence

$$
0 \rightarrow L_{1} \operatorname{SP}^{2}(A) \rightarrow L_{1} \Gamma_{2}(A) \rightarrow \operatorname{Tor}(A, \mathbb{Z} / 2) \rightarrow 0
$$

Analogous short exact sequences were obtained by Jean in [30, Section 3.1] for the functor $\Gamma_{3}$ :

$$
\begin{aligned}
& 0 \rightarrow L_{1} \operatorname{SP}^{3}(A) \rightarrow L_{1} \Gamma_{3}(A) \rightarrow(\operatorname{Tor}(A, \mathbb{Z} / 2) \otimes A \otimes \mathbb{Z} / 2) \oplus \operatorname{Tor}(A, \mathbb{Z} / 3) \rightarrow 0 \\
& 0 \rightarrow L_{2} \operatorname{SP}^{3}(A) \rightarrow L_{2} \Gamma_{3}(A) \rightarrow \operatorname{Tor}(A, \mathbb{Z} / 2) \otimes \operatorname{Tor}(A, \mathbb{Z} / 2) \rightarrow 0
\end{aligned}
$$

\subsection{Koszul complexes}

Let $f: P \rightarrow Q$ be a homomorphism of abelian groups. For $n \geq 1$ and any $k=0, \ldots$, $n-1$ consider the maps

$$
\kappa_{k+1}: \Lambda^{k+1}(P) \otimes \mathrm{SP}^{n-k-1}(Q) \rightarrow \Lambda^{k}(P) \otimes \mathrm{SP}^{n-k}(Q)
$$

defined, for $p_{i} \in P$ and $q_{j} \in Q$, by

$$
\begin{aligned}
\kappa_{k+1}: p_{1} \wedge \cdots \wedge p_{k+1} & \otimes q_{k+2} \cdots q_{n} \\
& \mapsto \sum_{i=1}^{k+1}(-1)^{k+1-i} p_{1} \wedge \cdots \wedge \hat{p}_{i} \wedge \cdots \wedge p_{k+1} \otimes f\left(p_{i}\right) q_{k+2} \cdots q_{n}
\end{aligned}
$$

The associated Koszul complex is defined by

(2-28) $\operatorname{Kos}_{n}(f): 0 \rightarrow \Lambda^{n}(P) \stackrel{\kappa_{n}}{\rightarrow} \bigwedge^{n-1}(P) \otimes Q$

$$
\stackrel{\kappa_{n-1}}{\rightarrow} \cdots \rightarrow P \otimes \mathrm{SP}^{n-1}(Q) \stackrel{\kappa_{1}}{\rightarrow} \mathrm{SP}^{n}(Q) \rightarrow 0
$$

Dually, one defines maps

$$
\kappa^{k+1}: \Gamma_{k+1}(P) \otimes \Lambda^{n-k-1}(Q) \rightarrow \Gamma_{k}(P) \otimes \Lambda^{n-k}(Q), \quad k=0, \ldots, n-1
$$

by setting

$$
\begin{aligned}
\kappa^{k+1}: \gamma_{r_{1}}\left(p_{1}\right) \cdots \gamma_{r_{k}}\left(p_{k}\right) \otimes q_{1} \wedge \cdots \wedge q_{n-k-1} \\
\\
\mapsto \sum_{j=1}^{k} \gamma_{r_{1}}\left(p_{1}\right) \cdots \gamma_{r_{j-1}}\left(p_{j}\right) \cdots \gamma_{r_{k}}\left(p_{k}\right) \otimes f\left(p_{j}\right) \wedge q_{1} \wedge \cdots \wedge q_{n-k-1}
\end{aligned}
$$


These maps determine a dual Koszul complex:

$(2-30) \operatorname{Kos}^{n}(f): 0 \rightarrow \Gamma_{n}(P) \stackrel{\kappa^{n}}{\rightarrow} \Gamma_{n-1}(P) \otimes Q^{\kappa^{n-1}} \rightarrow \cdots \rightarrow P \otimes \Lambda^{n-1}(Q) \stackrel{\kappa^{1}}{\rightarrow} \Lambda^{n}(Q) \rightarrow 0$

The complexes $\operatorname{Kos}_{n}(f)$ and $\operatorname{Kos}^{n}(f)$ are the total degree $n$ components of the Koszul complexes $\Lambda(P) \otimes \operatorname{SP}(Q)$ and $\Gamma(P) \otimes \Lambda(Q)$ associated to a given homomorphism $f: P \rightarrow Q$. For a two-term flat resolution (2-15) of an abelian group $A$, the complexes $\operatorname{Kos}_{n}(f)$ and $\operatorname{Kos}^{n}(f)$ represent the derived category objects $L \operatorname{SP}^{n}(A)$ and $L \Lambda^{n}(A)$ respectively (see for example Köck [31]). In particular, when $P$ is free abelian and $f$ is the identity map, both these complexes are acyclic.

For $n \geq 2$, the derived category object $L \mathrm{SP}^{n-1}(A) \stackrel{L}{\otimes} A$ may be represented, for a given 2-term flat resolution $f: L \rightarrow M$ of $A$, by the tensor product of $\operatorname{Kos}_{n}(f)$ and $L \rightarrow M$, in other words as the total complex associated to the bicomplex:

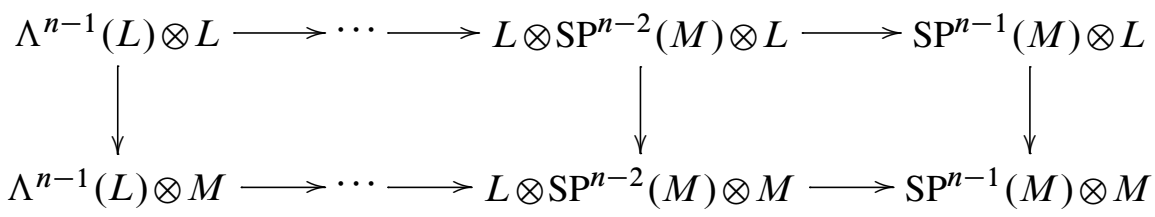

The diagram $L J^{n}(A) \rightarrow L \mathrm{SP}^{n-1}(A) \stackrel{L}{\otimes} A \rightarrow L \mathrm{SP}^{n}(A)$ in the derived category may therefore be represented by the following diagram of (horizontal) complexes:

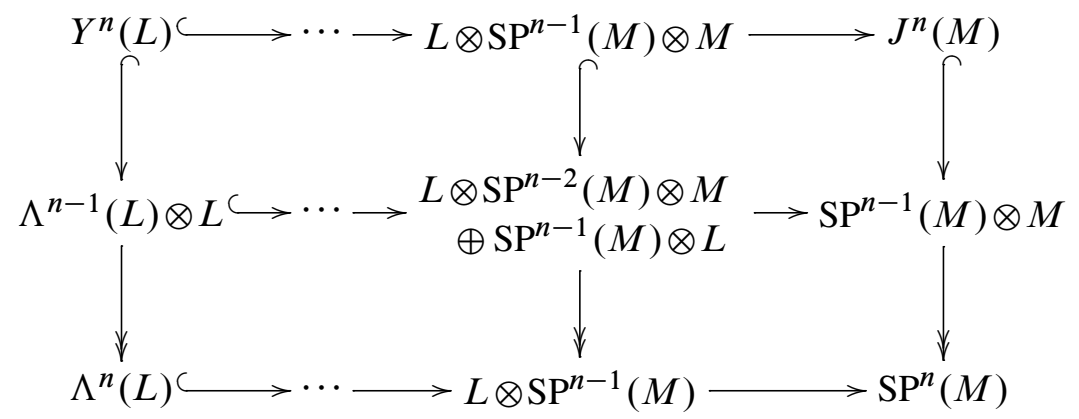

The upper line

$$
Y^{n}(L) \longrightarrow \cdots \longrightarrow L \otimes \operatorname{SP}^{n-1}(M) \otimes M \longrightarrow J^{n}(M)
$$

in this diagram is a Koszul complex for the functors $J^{n}$ and $Y^{n}$. A similar diagram, whose lower line is the dual Koszul complex (2-30) for $n=3$ is described in 
Appendix A. For Koszul complexes associated to more general Schur functors, see Lascoux [32, Lemma 1.9.1] and Akin, Buchsbaum and Weyman [1].

\subsection{Pensions and décalage}

Consider the homomorphisms

$$
\begin{aligned}
& \eta_{n}: \Lambda^{n}(A) \otimes \Lambda^{n}(B) \rightarrow \mathrm{SP}^{n}(A \otimes B), \\
& v_{n}: \Gamma_{n}(A) \otimes \operatorname{SP}^{n}(B) \rightarrow \operatorname{SP}^{n}(A \otimes B)
\end{aligned}
$$

as in Bousfield [7, 7.4] and Akin, Buchsbaum and Weyman [1]. These are characterized as the unique homomorphisms for which the corresponding diagrams

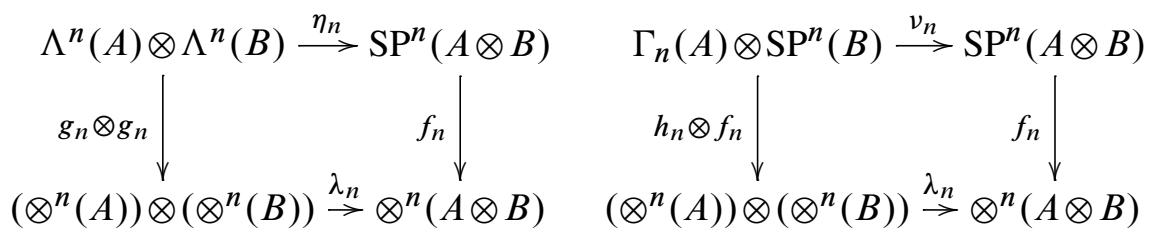

commute, where $\lambda_{n}$ is defined by

$$
\lambda_{n}:\left(a_{1} \otimes \cdots \otimes a_{n}\right) \otimes\left(b_{1} \otimes \cdots \otimes b_{n}\right) \mapsto\left(a_{1} \otimes b_{1}\right) \otimes \cdots \otimes\left(a_{n} \otimes b_{n}\right), \quad a_{i} \in A, \quad b_{i} \in B .
$$

The map $\eta_{n}$ is given by the formula

$$
\left(a_{1} \wedge \cdots \wedge a_{n}\right) \otimes\left(b_{1} \wedge \cdots \wedge b_{n}\right) \mapsto \sum_{\sigma \in \Sigma_{n}} \operatorname{sign}(\sigma)\left(a_{1} \otimes b_{\sigma(1)}\right) \cdots\left(a_{n} \otimes b_{\sigma(n)}\right)
$$

and $v_{n}$ satisfies

$$
v_{n}: \gamma_{n}(a) \otimes b_{1} \cdots b_{n} \mapsto\left(a \otimes b_{1}\right) \cdots\left(a \otimes b_{n}\right) .
$$

Formula (2-35) makes it clear that the composite arrow

$$
\mathbb{Z}[A] \otimes \operatorname{SP}^{n}(B) \longrightarrow \Gamma_{n}(A) \otimes \operatorname{SP}^{n}(B) \longrightarrow \operatorname{SP}^{n}(A \otimes B)
$$

is the obvious one.

The maps $\eta_{n}$ and $v_{n}$ induce natural isomorphisms

$$
\begin{aligned}
& L \Lambda^{n}(A, m) \stackrel{L}{\otimes} \Lambda^{n}(\mathbb{Z}, 1) \simeq L \operatorname{SP}^{n}(A, m+1) \\
& L \Gamma_{n}(A, m) \stackrel{L}{\otimes} \operatorname{SP}^{n}(\mathbb{Z}, 2) \simeq L \operatorname{SP}^{n}(A, m+2) .
\end{aligned}
$$


These derived pairings determine for all $n \geq 1$, by adjunction with the volume element $n-$ cycle in $\Lambda^{n}(\mathbb{Z}, 1)_{n}=\Lambda^{n}\left(\mathbb{Z}^{n}\right)$ and the corresponding element in $\mathrm{SP}^{n}(\mathbb{Z}, 2)$ respectively, a pair of functorial pension morphisms

$$
\begin{aligned}
& L \Lambda^{j}(A, n)[j] \longrightarrow L \operatorname{SP}^{j}(A, n+1) \\
& L \Gamma^{j}(A, n)[j] \longrightarrow L \Lambda^{j}(A, n+1)
\end{aligned}
$$

in the derived category. These maps induce isomorphisms on homotopy groups

$$
\begin{aligned}
& L_{i} \Lambda^{j}(A, n) \stackrel{\simeq}{\longrightarrow} L_{i+j} \operatorname{SP}^{j}(A, n+1) \\
& L_{i} \Gamma_{j}(A, n) \stackrel{\simeq}{\longrightarrow} L_{i+j} \Lambda^{j}(A, n+1) .
\end{aligned}
$$

(see Bousfield [8]). The inverses of the maps (2-39) and (2-40) are iterated boundary maps arising from the exactness of the Koszul complexes (see Quillen [43] and Illusie [29, I 4.3.2]), and are known as décalage isomorphisms. By composition, the maps (2-39) and (2-40) determine a family of double décalage isomorphism

$$
L_{i} \Gamma_{j}(A, n) \simeq L_{i+2 j} \mathrm{SP}^{j}(A, n+2) .
$$

Similarly, the existence of Koszul sequences of type (2-32) determine for all $j, n \geq 0$ décalage isomorphisms

$$
L_{i} Y^{j}(A, n) \simeq L_{i+j} J^{j}(A, n+1)
$$

between the derived functor of $J^{j}$ and $Y^{j}$.

\section{The de Rham complex and its dual}

Let $A$ be an abelian group. For $n \geq 1$, let $D_{*}^{n}(A)$ and $C_{*}^{n}(A)$ be the complexes of abelian groups defined by

$$
\begin{array}{ll}
D_{i}^{n}(A)=\operatorname{SP}^{i}(A) \otimes \Lambda^{n-i}(A), & 0 \leq i \leq n, \\
C_{i}^{n}(A)=\Lambda^{i}(A) \otimes \Gamma_{n-i}(A), & 0 \leq i \leq n,
\end{array}
$$

where the differentials $d^{i}: D_{i}^{n}(A) \rightarrow D_{i-1}^{n}(A)$ and $d_{i}: C_{i}^{n}(A) \rightarrow C_{i-1}^{n}(A)$ are

$$
\begin{gathered}
d^{i}\left(\left(b_{1} \cdots b_{i}\right) \otimes b_{i+1} \wedge \cdots \wedge b_{n}\right)=\sum_{k=1}^{i}\left(b_{1} \cdots \hat{b}_{k} \cdots b_{i}\right) \otimes b_{k} \wedge b_{i+1} \wedge \cdots \wedge b_{n}, \\
d_{i}\left(b_{1} \wedge \cdots \wedge b_{i} \otimes X\right)=\sum_{k=1}^{i}(-1)^{k} b_{1} \wedge \cdots \wedge \hat{b}_{k} \wedge \cdots \wedge b_{i} \otimes b_{k} X
\end{gathered}
$$


for any $X \in \Gamma_{n-i}(A)$. The complex $D^{n}(A)$ is the degree $n$ component of the classical de Rham complex, first introduced in the present context of polynomial functors by Franjou, Lannes and Schwarz [24] and denoted $\Omega_{n}$ by Franjou [23]. The dual complexes $C^{n}(A)$ were considered by Jean [30]. We will call them the dual de Rham complexes.

We will now give a functorial description of certain homology groups of these complexes $C^{n}(A)$.

Proposition 3.1 Let $A$ be a free abelian.

(1) (Franjou [23]) For any prime number $p$, we have $H_{0} C^{p}(A)=A \otimes \mathbb{Z} / p$, and $H_{i} C^{p}(A)=0$, for all $i>0$.

(2) Jean [30] There is a natural isomorphism

$$
H_{0} C^{n}(A) \simeq \bigoplus_{p \mid n, p \text { prime }} \Gamma_{n / p}(A \otimes \mathbb{Z} / p)
$$

A proof of Proposition 3.1 (2) is given by one of us in Mikhailov [39].

The higher homology groups $H_{i} C^{n}(A)$ are more complicated. Table 1 below, which is a consequence of the main theorem in [39], gives a complete description of $H_{i} C^{n}(A)$ for $n \leq 7$ and $A$ free abelian.

\begin{tabular}{c|cccc}
$n$ & $H_{0} C^{n}(A)$ & $H_{1} C^{n}(A)$ & $H_{2} C^{n}(A)$ & $H_{3} C^{n}(A)$ \\
\hline 8 & $\Gamma_{4}(A \otimes \mathbb{Z} / 2)$ & $*$ & $*$ & $*$ \\
7 & $A \otimes \mathbb{Z} / 7$ & 0 & 0 & 0 \\
6 & $\Gamma_{2}(A \otimes \mathbb{Z} / 3)$ & $\Lambda^{2}(A \otimes \mathbb{Z} / 3)$ & $\Lambda^{3}(A \otimes \mathbb{Z} / 2)$ & 0 \\
5 & $\oplus \Gamma_{3}(A \otimes \mathbb{Z} / 2)$ & $\oplus \mathscr{L}^{3}(A \otimes \mathbb{Z} / 2)$ & 0 & 0 \\
4 & $A \otimes \mathbb{Z} / 5$ & 0 & 0 & 0 \\
3 & $\Gamma_{2}(A \otimes \mathbb{Z} / 2)$ & $\Lambda^{2}(A \otimes \mathbb{Z} / 2)$ & 0 & 0 \\
2 & $A \otimes \mathbb{Z} / 3$ & 0 & 0 & 0
\end{tabular}

Table 1

For example, the isomorphism

$$
f: \Lambda^{2}(A \otimes \mathbb{Z} / 2) \rightarrow H_{1} C^{4}(A)
$$

is defined, for representatives $a, b \in A$ of $\bar{a}, \bar{b} \in A \otimes \mathbb{Z} / 2$, by

$$
f: \bar{a} \otimes \bar{b} \mapsto a \otimes a \gamma_{2}(b)-b \otimes b \gamma_{2}(a) .
$$




\subsection{Comparing the de Rham and Koszul complexes}

For any free abelian group $A$, consider the following natural monomorphism of complexes $\operatorname{Kos}_{n}(A) \longrightarrow C^{n}(A)$ :

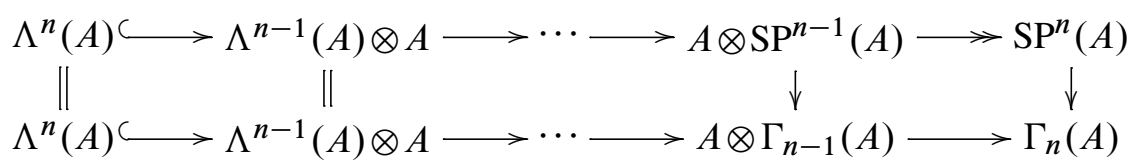

Let us denote the cokernel of this map by $D^{n}(A)$. We set

$$
W_{n}(A)=\operatorname{coker}\left\{\operatorname{SP}^{n}(A) \rightarrow \Gamma_{n}(A)\right\}
$$

so that the complex $D^{n}(A)$ is

$$
\Lambda^{n-2}(A) \otimes W_{2}(A) \rightarrow \Lambda^{n-3}(A) \otimes W_{3}(A) \rightarrow \cdots \rightarrow A \otimes W_{n-1}(A) \rightarrow W_{n}(A) .
$$

Since the Koszul complex $\operatorname{Kos}_{n}(A)$ is acyclic, it follows that

$$
H_{i} C^{n}(A) \simeq H_{i} D^{n}(A) \text { for all } n \geq 0 .
$$

Proposition 3.1 implies in particular that the sequence

$$
0 \rightarrow A \otimes A \otimes \mathbb{Z} / 2 \rightarrow W_{3}(A) \rightarrow A \otimes \mathbb{Z} / 3 \rightarrow 0
$$

is exact (and splits naturally). For every $n \geq 2, m \geq 0$, we obtain the natural exact sequence:

$$
0 \rightarrow L \operatorname{SP}^{n}(A, m) \rightarrow L \Gamma_{n}(A, m) \rightarrow L W_{n}(A, m) \rightarrow 0
$$

Passing to homotopy groups and applying the décalage isomorphisms (2-39), (2-40), this yields the long exact sequence:

$$
\begin{aligned}
\cdots \rightarrow L_{i} & \operatorname{SP}^{n}(A, m) \rightarrow L_{i+2 n} \operatorname{SP}^{n}(A, m+2) \rightarrow L_{i} W_{n}(A, m) \\
& \rightarrow L_{i-1} \operatorname{SP}^{n}(A, m) \rightarrow L_{i+2 n} \operatorname{SP}^{n}(A, m+2) \rightarrow L_{i-1} W_{n}(A, m) \rightarrow \cdots
\end{aligned}
$$

Let $X$ be a simplicial free abelian group and $k \geq 1, n \geq 2$ a pair of integers. If $\pi_{i}(X)=$ 0 for all $i<k$, then by Dold and Puppe [20, Satz 12.1]

$$
\pi_{i}\left(\operatorname{SP}^{n}(X)\right)=0 \begin{cases}\text { for } i<n & \text { when } k=1, \\ \text { for } i<k+2 n-2 & \text { when } k>1 .\end{cases}
$$

We will make use of exact sequence (3-5) and of the assertion (3-6) when we compute certain derived functors of low degree polynomial functors. 


\section{Derived functors of quadratic functors}

For every abelian group $A$, the exactness of the sequence (2-26) implies that $W_{2}(A) \simeq$ $A \otimes \mathbb{Z} / 2$. Since this functor is additive in $A$, it follows immediately that

$$
L_{i} W_{2}(A, m) \simeq \begin{cases}A \otimes \mathbb{Z} / 2 & i=m, \\ \operatorname{Tor}(A, \mathbb{Z} / 2) & i=m+1, \\ 0 & i \neq m, m+1,\end{cases}
$$

for all $m$. Let us define a new functor $\lambda^{2}(A)$ by

$$
\lambda^{2}(A):=\Lambda^{2}(A) \oplus \operatorname{Tor}(A, \mathbb{Z} / 2) .
$$

The long exact sequence (3-5), the connectivity result (3-6), and the décalage formulas (2-39) and (2-40) produce the following complete description of the derived functors of the second symmetric power functor $\mathrm{SP}^{2}$ :

\section{Proposition 4.1}

$$
L_{i} \operatorname{SP}^{2}(A, n)= \begin{cases}\mathrm{SP}^{2}(A) & i=0, n=0 \\ \mathscr{S}_{2}(A) & i=1, n=0 \\ \Lambda^{2}(A) & i=2, n=1 \\ A \otimes \mathbb{Z} / 2 & i=n+2, n+4, \ldots, n+2\left[\frac{n-1}{2}\right], \\ \operatorname{Tor}(A, \mathbb{Z} / 2) & i=n+3, n+5, \ldots, n+2\left[\frac{n-1}{2}\right]+1, i \neq 2 n \\ \Gamma_{2}(A) & i=2 n, n \neq 0 \text { even, } \\ \lambda^{2}(A) & i=2 n, n \neq 1 \text { odd, } \\ R_{2}(A) & i=2 n+1, n \neq 0 \text { even, } \\ \Omega_{2}(A) & i=2 n+1, n \text { odd }, \\ 0 & \text { for all other } i\end{cases}
$$

We will only sketch the proof of this computation in the present quadratic situation, and will discuss the more elaborate case of cubical functors in the following section. These quadratic results were also obtained in Baues and Pirashvili [5] by a different method (see also Mikhailov and Passi [40, A.15]).

Proof The first two equations in the statement of Proposition 4.1 follow from the definitions. By double décalage (2-41), there is an iterated isomorphism

$$
\Gamma_{2}(A)=L_{0} \Gamma_{2}(A, 0) \simeq L_{4} \operatorname{SP}^{2}(A, 2)
$$


which determines the sixth equation in (4-2) for $n=2$. The general case of the sixth equation then follows by induction when we consider the isomorphism $L_{2 n} \mathrm{SP}^{2}(A, n) \simeq$ $L_{2 n+2} \mathrm{SP}^{2}(A, n+2)$ from (3-5) for $n$ even. Décalage also implies that

$$
\Lambda^{2} A \simeq L_{2} \operatorname{SP}^{2}(A, 1)
$$

and the sequence (3-4) then determines a short exact sequence

$$
0 \rightarrow L_{2} \operatorname{SP}^{2}(A, 1) \rightarrow L_{2} \Gamma_{2}(A, 1) \rightarrow \operatorname{Tor}(A, \mathbb{Z} / 2) \rightarrow 0
$$

Consider the following diagram, in which the vertical arrows are the suspension maps:

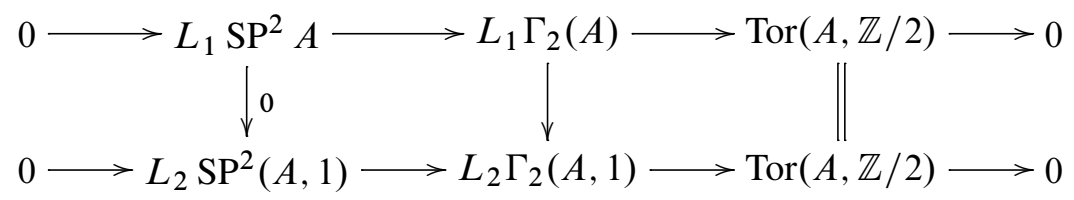

The left-hand vertical arrow is trivial by Dold and Puppe [20, Korollar 6.6], since all elements of $L_{1} \mathrm{SP}^{2} A$ are in the image of the arrow (2-19) for $n=2$. The lower sequence is therefore split since it is the pushout of the upper one by the trivial map, and a diagram chase makes it clear that this splitting is functorial. This proves the seventh equation in Proposition 4.1 for $n=3$ by the double décalage isomorphism

$$
L_{2} \Gamma_{2}(A, 1) \simeq L_{6} \operatorname{SP}^{2}(A, 3)
$$

The general case of the seventh equation now follows by induction since (3-4) and décalage imply that

$$
L_{i} \operatorname{SP}^{2}(A, n) \simeq L_{i} \Gamma_{2}(A, n) \simeq L_{i+4} \operatorname{SP}^{2}(A, n+2)
$$

for all $n$. A similar discussion, in the next degree, shows that the eight and ninth equations are also satisfied. The remaining fourth and fifth equations are proved by considering once more the sequence (3-5), and observing that the functors $L_{i} \operatorname{SP}^{2}(A, n)$ vanish by [20] whenever $i$ is sufficiently large.

As a corollary, one finds that this computation (and even the inductive reasoning that led to it) can be carried over by the décalage isomorphisms (2-39), (2-41) to the derived 
functors of $\Lambda^{2}$ and $\Gamma_{2}$. We simply state the result:

$$
L_{i} \Lambda^{2}(A, n)= \begin{cases}\Lambda^{2}(A) & i=0, n=0, \\ A \otimes \mathbb{Z} / 2 & i=n+1, n+3, \ldots, n+2\left[\frac{n-1}{2}\right]+1, i \neq 2 n, \\ \operatorname{Tor}(A, \mathbb{Z} / 2) & i=n+2, n+4, \ldots, n+2\left[\frac{n-1}{2}\right], \\ \Gamma_{2}(A) & i=2 n, n \text { odd, } \\ \lambda^{2}(A) & i=2 n, n \neq 0 \text { even, } \\ R_{2}(A) & i=2 n+1, n \text { odd, } \\ \Omega_{2}(A) & i=2 n+1, n \text { even, } \\ 0 & \text { for all other } i . \\ L_{i} \Gamma_{2}(A, n)= \begin{cases}A \otimes \mathbb{Z} / 2 & i=n, n+2, \ldots, n+2\left[\frac{n-1}{2}\right], n>0, \\ \operatorname{Tor}(A, \mathbb{Z} / 2) & i=n+1, n+3, \ldots, n+2\left[\frac{n-1}{2}\right]+1, \\ \Gamma_{2}(A) & i=2 n, n \text { even, } \\ \lambda^{2}(A) & i=2 n, n \text { odd, } \\ R_{2}(A) & i=2 n+1, n \text { even, } \\ \Omega_{2}(A) & i=2 n+1, n \text { odd, } \\ 0 & \text { for all other } i .\end{cases} \end{cases}
$$

\section{The derived functors of certain cubical functors}

It follows from (3-3) that

$$
W_{3}(A)=(A \otimes A \otimes \mathbb{Z} / 2) \oplus(A \otimes \mathbb{Z} / 3),
$$

and this derives to an isomorphism

$$
L W_{3}(A) \simeq(A \stackrel{L}{\otimes} A \stackrel{L}{\otimes} \mathbb{Z} / 2) \oplus(A \stackrel{L}{\otimes} \mathbb{Z} / 3)
$$

in the derived category, from which the values of $L_{i} W_{3}(A)$ follow immediately (consistently with the two equations (2-27)). This implies that

$$
L_{i} W_{3}(A, 1)= \begin{cases}A \otimes \mathbb{Z} / 3 & i=1, \\ A \otimes A \otimes \mathbb{Z} / 2 \oplus \operatorname{Tor}(A, \mathbb{Z} / 3) & i=2, \\ \operatorname{Tor}_{1}(A, A, \mathbb{Z} / 2) & i=3, \\ \operatorname{Tor}_{2}(A, A, \mathbb{Z} / 2) & i=4, \\ 0 & \text { for all other } i,\end{cases}
$$


and for $n>1$,

$$
L_{i} W_{3}(A, n)= \begin{cases}A \otimes \mathbb{Z} / 3 & i=n, \\ \operatorname{Tor}(A, \mathbb{Z} / 3) & i=n+1, \\ A \otimes A \otimes \mathbb{Z} / 2 & i=2 n, \\ \operatorname{Tor}_{1}(A, A, \mathbb{Z} / 2) & i=2 n+1, \\ \operatorname{Tor}_{2}(A, A, \mathbb{Z} / 2) & i=2 n+2, \\ 0 & \text { for all other } i .\end{cases}
$$

Consider the map

$$
L \mathrm{SP}^{3}(A, n) \otimes \mathbb{Z}[K(\mathbb{Z}, 2)] \longrightarrow L \mathrm{SP}^{3}(A, n+2)
$$

obtained from the pairing (2-36). The homology class $\gamma_{3}\left(i_{2}\right) \in \Gamma_{3}(\mathbb{Z}) \simeq H_{6}(K(\mathbb{Z}, 2))$ determines by adjunction a family of pension maps

$$
\epsilon_{3}: L_{i} \operatorname{SP}^{3}(A, n) \rightarrow L_{i+6} \operatorname{SP}^{3}(A, n+2),
$$

which factor through the décalage isomorphisms (2-41):

$$
L_{i} \operatorname{SP}^{3}(A, n) \rightarrow L_{i} \Gamma_{3}(A, n) \stackrel{\sim}{\longrightarrow} L_{i+6} \mathrm{SP}^{3}(A, n+2) .
$$

These maps $\epsilon_{3}$ are isomorphisms for $i \neq n-1, n, n+1, n+2,2 n-1,2 n, 2 n+1,2 n+2$. In addition the sequence

$$
\begin{aligned}
0 & \rightarrow L_{2 n+2} \operatorname{SP}^{3}(A, n) \rightarrow L_{2 n+8} \operatorname{SP}^{3}(A, n+2) \rightarrow \operatorname{Tor}_{2}(A, A, \mathbb{Z} / 2) \\
& \rightarrow L_{2 n+1} \operatorname{SP}^{3}(A, n) \rightarrow L_{2 n+7} \operatorname{SP}^{3}(A, n+2) \rightarrow \operatorname{Tor}_{1}(A, A, \mathbb{Z} / 2) \\
& \rightarrow L_{2 n} \operatorname{SP}^{3}(A, n) \rightarrow L_{2 n+6} \operatorname{SP}^{3}(A, n+2) \rightarrow A \otimes A \otimes \mathbb{Z} / 2 \\
& \rightarrow L_{2 n-1} \mathrm{SP}^{3}(A, n) \rightarrow L_{2 n+5} \operatorname{SP}^{3}(A, n+2)
\end{aligned}
$$

is exact by (3-5). Furthermore, for $n>1$,

$$
\begin{aligned}
& L_{n+7} \operatorname{SP}^{3}(A, n+2) \simeq \operatorname{Tor}(A, \mathbb{Z} / 3) \\
& L_{n+6} \operatorname{SP}^{3}(A, n+2) \simeq A \otimes \mathbb{Z} / 3
\end{aligned}
$$

by (3-6). Finally, according to Bousfield [8, Corollary 4.3], the maps (5-5) are split injections for all $i \geq 0$ and all $n>1$. The long exact sequence (5-6) therefore decomposes for each $n>1$ into short exact sequences:

$$
\begin{aligned}
& 0 \rightarrow L_{2 n+2} \mathrm{SP}^{3}(A, n) \rightarrow L_{2 n+8} \mathrm{SP}^{3}(A, n+2) \rightarrow \operatorname{Tor}_{2}(A, A, \mathbb{Z} / 2) \rightarrow 0 \\
& 0 \rightarrow L_{2 n+1} \mathrm{SP}^{3}(A, n) \rightarrow L_{2 n+7} \mathrm{SP}^{3}(A, n+2) \rightarrow \operatorname{Tor}_{1}(A, A, \mathbb{Z} / 2) \rightarrow 0 \\
& 0 \rightarrow L_{2 n} \mathrm{SP}^{3}(A, n) \rightarrow L_{2 n+6} \mathrm{SP}^{3}(A, n+2) \rightarrow A \otimes A \otimes \mathbb{Z} / 2 \rightarrow 0
\end{aligned}
$$


and an isomorphism

$$
L_{2 n+5} \operatorname{SP}^{3}(A, n+2) \simeq L_{2 n-1} \operatorname{SP}^{3}(A, n) .
$$

Example 5.1 Since the values taken by the derived functors of $W_{3}$ in (5-3) and (5-4) are distinct, we must consider the implications of (5-3) separately. Observe that exact sequence (3-5) and the equations (5-3) imply that

$$
\begin{aligned}
L_{11} \operatorname{SP}^{3}(A, 3) & =\Omega_{3}(A), \\
L_{8} \operatorname{SP}^{3}(A, 3) & =A \otimes A \otimes \mathbb{Z} / 2 \oplus \operatorname{Tor}(A, \mathbb{Z} / 3), \\
L_{7} \operatorname{SP}^{3}(A, 3) & =A \otimes \mathbb{Z} / 3,
\end{aligned}
$$

and that the groups $L_{i} \mathrm{SP}^{3}(A, 3)$ for $i=9,10$ live in the long exact sequence

$$
\begin{aligned}
0 \rightarrow L_{1} \Lambda^{3}(A) \rightarrow L_{10} & \mathrm{SP}^{3}(A, 3) \rightarrow \operatorname{Tor}_{2}(A, A, \mathbb{Z} / 2) \\
& \stackrel{\partial}{\rightarrow} \Lambda^{3}(A) \stackrel{\varepsilon_{3}}{\rightarrow} L_{9} \mathrm{SP}^{3}(A, 3) \rightarrow \operatorname{Tor}_{1}(A, A, \mathbb{Z} / 2) \rightarrow 0 .
\end{aligned}
$$

The diagram

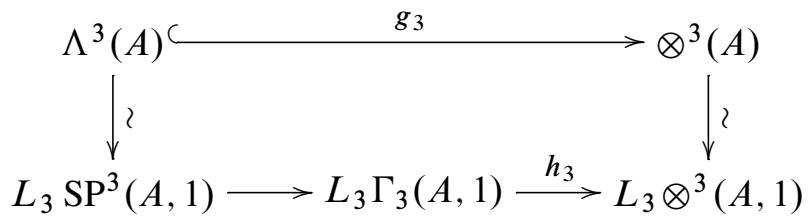

commutes, where the left-hand vertical arrow is the map (2-39), and the right-hand one the corresponding obvious décalage map for tensor powers. It follows that the composite map

$$
\Lambda^{3} A \stackrel{\sim}{\longrightarrow} L_{3} \operatorname{SP}^{3}(A, 1) \longrightarrow L_{3} \Gamma_{3}(A, 1) \stackrel{\varepsilon_{3}}{\sim} L_{9} \operatorname{SP}^{3}(A, 3)
$$

is injective so that the boundary map $\partial$ in (5-7) is trivial. The complete description of the $L_{i} \mathrm{SP}^{3}(A, 3)$ is therefore given by

$$
L_{i} \operatorname{SP}^{3}(A, 3)= \begin{cases}\Omega_{3}(A) & i=11, \\ A \otimes A \otimes \mathbb{Z} / 2 \oplus \operatorname{Tor}(A, \mathbb{Z} / 3) & i=8, \\ A \otimes \mathbb{Z} / 3 & i=7, \\ 0 & i \neq 7,8,9,10,11,\end{cases}
$$

and the exactness of the sequences

$$
\begin{aligned}
0 \rightarrow L_{1} \Lambda^{3}(A) & \rightarrow L_{10} \mathrm{SP}^{3}(A, 3) \rightarrow \operatorname{Tor}_{2}(A, A, \mathbb{Z} / 2) \rightarrow 0 \\
0 & \rightarrow \Lambda^{3}(A) \rightarrow L_{9} \operatorname{SP}^{3}(A, 3) \rightarrow \operatorname{Tor}_{1}(A, A, \mathbb{Z} / 2) \rightarrow 0 .
\end{aligned}
$$


The discussion in Example 5.1 also applies to the derived functors $L_{i} \operatorname{SP}^{3}(A, n)$ for other values of $n$. Here is the complete description of these functors:

Theorem 5.2 Case I $n \geq 3$ is odd.

$$
L_{i} \operatorname{SP}^{3}(A, n)= \begin{cases}A \otimes \mathbb{Z} / 3 & n+4 \leq i<2 n+2, i-n \equiv 0 \bmod 4, \\ \operatorname{Tor}(A, \mathbb{Z} / 3) & n+4 \leq i<2 n+2, i-n \equiv 1 \bmod 4, \\ A \otimes A \otimes \mathbb{Z} / 2 & i=2 n+2, n \equiv 1 \bmod 4, \\ \operatorname{Tor}(A, \mathbb{Z} / 3) \oplus A \otimes A \otimes \mathbb{Z} / 2 & i=2 n+2, n \equiv 3 \bmod 4, \\ \operatorname{Tor}_{1}(A, A, \mathbb{Z} / 2) & 2 n+3 \leq i \leq 3 n-2, i-n \equiv 2 \bmod 4, \\ A \otimes \mathbb{Z} / 3 \oplus \operatorname{Tor}_{1}(A, A, \mathbb{Z} / 2) & 2 n+3 \leq i \leq 3 n-2, i-n \equiv 0 \bmod 4, \\ \Omega_{3}(A) & i=3 n+2, \\ 0 & \text { for all other } i .\end{cases}
$$

In addition, the following sequences are exact:

$$
\begin{aligned}
& \begin{aligned}
0 \rightarrow \operatorname{Tor}(A, \mathbb{Z} / 3) \oplus A \otimes A \otimes \mathbb{Z} / 2 \rightarrow L_{i} \operatorname{SP}^{3}(A, 3) \rightarrow \operatorname{Tor}_{2}(A, A, \mathbb{Z} / 2) \rightarrow 0, \\
2 n+4 \leq i \leq 3 n-1, i-n \equiv 1 \bmod 4,
\end{aligned} \\
& \begin{aligned}
0 \rightarrow A \otimes A \otimes \mathbb{Z} / 2 \rightarrow L_{i} \operatorname{SP}^{3}(A, n) \rightarrow \operatorname{Tor}_{2}(A, A, \mathbb{Z} / 2) \rightarrow 0, \\
2 n+3 \leq i \leq 3 n-1, i-n \equiv 3 \bmod 4,
\end{aligned} \\
& 0 \rightarrow L_{1} \Lambda^{3}(A) \rightarrow L_{3 n+1} \operatorname{SP}^{3}(A, n) \rightarrow \operatorname{Tor}_{2}(A, A, \mathbb{Z} / 2) \rightarrow 0, \\
& 0 \rightarrow \Lambda^{3}(A) \rightarrow L_{3 n} \operatorname{SP}^{3}(A, n) \rightarrow \operatorname{Tor}_{1}(A, A, \mathbb{Z} / 2) \rightarrow 0 .
\end{aligned}
$$

Case II $n>3$ is even.

$$
L_{i} \operatorname{SP}^{3}(A, n)= \begin{cases}A \otimes \mathbb{Z} / 3 & n+4 \leq i<2 n+2, i-n \equiv 0 \bmod 4, \\ \operatorname{Tor}(A, \mathbb{Z} / 3) & n+4 \leq i<2 n+2, i-n \equiv 1 \bmod 4, \\ A \otimes A \otimes \mathbb{Z} / 2 & i=2 n+2, n \equiv 0 \bmod 4, \\ A \otimes \mathbb{Z} / 3 \oplus A \otimes A \otimes \mathbb{Z} / 2 & i=2 n+2, n \equiv 2 \bmod 4, \\ \operatorname{Tor}(A, \mathbb{Z} / 3) \oplus \operatorname{Tor}_{1}(A, A, \mathbb{Z} / 2) & 2 n+3 \leq i \leq 3 n-1, i-n \equiv 1 \bmod 4, \\ \operatorname{Tor}_{1}(A, A, \mathbb{Z} / 2) & 2 n+3 \leq i \leq 3 n-1, i-n \equiv 3 \bmod 4, \\ L_{1} \Gamma_{3}(A) & i=3 n+1, \\ R_{3}(A) & i=3 n+2, \\ 0 & \text { for all other } i .\end{cases}
$$


In addition, the following sequences are exact:

$$
\begin{gathered}
0 \rightarrow A \otimes \mathbb{Z} / 3 \oplus A \otimes A \otimes \mathbb{Z} / 2 \rightarrow L_{i} \operatorname{SP}^{3}(A, n) \rightarrow \operatorname{Tor}_{2}(A, A, \mathbb{Z} / 2) \rightarrow 0, \\
2 n+4 \leq i \leq 3 n-2, i-n \equiv 0 \bmod 4, \\
0 \rightarrow A \otimes A \otimes \mathbb{Z} / 2 \rightarrow L_{i} \operatorname{SP}^{3}(A, n) \rightarrow \operatorname{Tor}_{2}(A, A, \mathbb{Z} / 2) \rightarrow 0, \\
2 n+4 \leq i \leq 3 n-2, i-n \equiv 2 \bmod 4, \\
0 \rightarrow \Gamma_{3}(A) \rightarrow L_{3 n} \operatorname{SP}^{3}(A, n) \rightarrow \operatorname{Tor}_{2}(A, A, \mathbb{Z} / 2) \rightarrow 0 .
\end{gathered}
$$

The corresponding description of the derived functors of $\Lambda^{3}$ and $\Gamma_{3}$ now follows from the décalage isomorphisms (2-39) and (2-40).

\section{Some derived functors of $\mathrm{SP}^{4}$}

We will now make use of the computation of the homology of the dual de Rham complex $C^{4}(A)$ in Proposition 3.1 in order to investigate some of the derived functors of $\mathrm{SP}^{4}$. For $A$ a free abelian group, let us now consider the following diagram with exact rows and columns, which extends diagram (3-2) when $n=4$ :

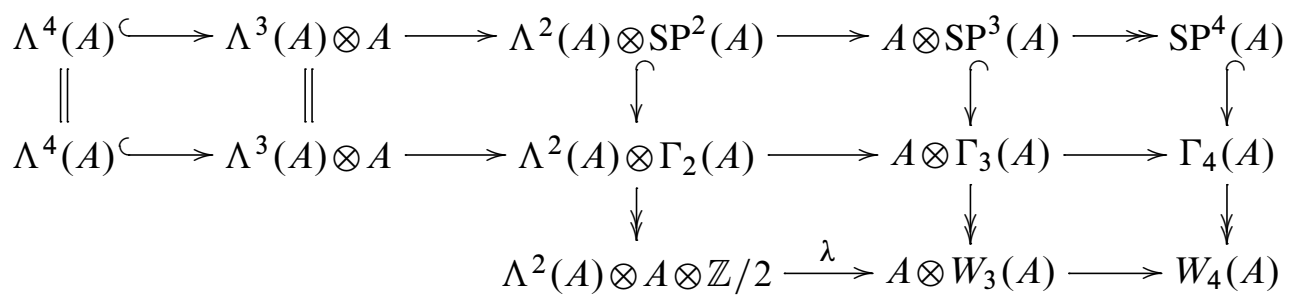

By Proposition 3.1, this determines a functorial diagram of exact sequences:

$(6-1)$

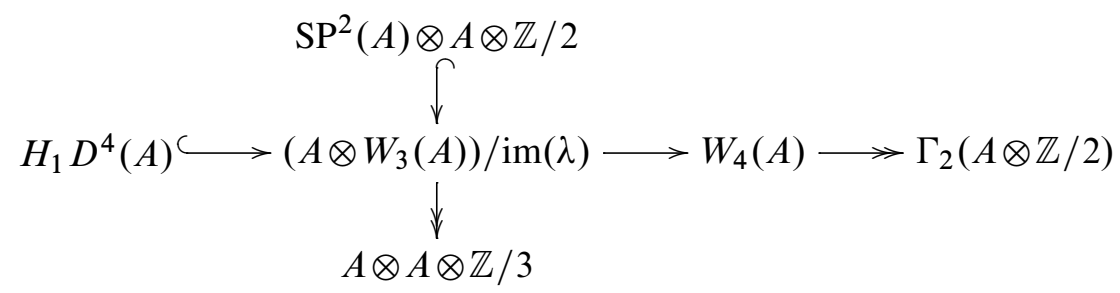

The map (3-1) defines canonical isomorphisms

$$
H_{1} D^{4}(A) \simeq H_{1} C^{4}(A) \simeq \Lambda^{2}(A \otimes \mathbb{Z} / 2) \simeq L_{1} \operatorname{SP}^{2}(A \otimes \mathbb{Z} / 2) .
$$

Let us define a map

$$
\delta: \Lambda^{2}(A \otimes \mathbb{Z} / 2) \rightarrow \mathrm{SP}^{2}(A) \otimes A \otimes \mathbb{Z} / 2\left(\subset\left(A \otimes W_{3}(A)\right) / \operatorname{im}(\lambda)\right)
$$


as follows, where $a, b$ are representatives in $A$ of the classes $\bar{a}$ and $\bar{b} \in A \otimes \mathbb{Z} / 2$ :

$$
\delta: \bar{a} \wedge \bar{b} \mapsto a a \otimes \bar{b}-b b \otimes \bar{a} .
$$

It follows from this discussion that diagram (6-1) induces a short exact sequence

$$
0 \rightarrow \frac{\operatorname{SP}^{2}(A) \otimes A \otimes \mathbb{Z} / 2}{\Lambda^{2}(A \otimes \mathbb{Z} / 2)} \oplus A \otimes A \otimes \mathbb{Z} / 3 \rightarrow W_{4}(A) \rightarrow \Gamma_{2}(A \otimes \mathbb{Z} / 2) \rightarrow 0 .
$$

The filtration on $\Gamma_{4}(A)$ provided by this description of $W_{4}(A)$ is consistent with that in Jean [30, Proposition 3.1.2]. Together with long exact sequence (3-5), it allows one to compute derived functors of the functor $\mathrm{SP}^{4}$ by comparing them to those of $\Gamma_{4}$ and taking into account the double décalage. For example, one finds

$$
\begin{aligned}
L_{9} \operatorname{SP}^{4}(A, 3) & \simeq A \otimes \mathbb{Z} / 2, \\
L_{10} \operatorname{SP}^{4}(A, 3) & \simeq A \otimes A \otimes \mathbb{Z} / 3 \oplus \Lambda^{2}(A \otimes \mathbb{Z} / 2) \oplus \operatorname{Tor}(A, \mathbb{Z} / 2), \\
L_{10} \operatorname{SP}^{4}(A, 4) & \simeq A \otimes \mathbb{Z} / 2, \\
L_{11} \operatorname{SP}^{4}(A, 4) & \simeq \operatorname{Tor}(A, \mathbb{Z} / 2) .
\end{aligned}
$$

\section{Lie and super-Lie functors}

We will now consider the structure theory of Lie and super-Lie functors.

\subsection{The third Lie functor}

For any free abelian group $A$, consider the Koszul resolution

$$
0 \rightarrow \Lambda^{3}(A) \rightarrow \Lambda^{2}(A) \otimes A \stackrel{f}{\rightarrow} A \otimes \mathrm{SP}^{2}(A) \rightarrow \mathrm{SP}^{3}(A) \rightarrow 0,
$$

in which the map $f$ is defined by

$$
f: a \wedge b \otimes c \mapsto a \otimes b c-b \otimes a c, \quad a, b, c \in A .
$$

This map decomposes as

$$
\Lambda^{2}(A) \otimes A \stackrel{u}{\longrightarrow} A \otimes A \otimes A \stackrel{v}{\longrightarrow} A \otimes \operatorname{SP}^{2}(A)
$$

where

$$
\begin{aligned}
& u: a \wedge b \otimes c \mapsto a \otimes b \otimes c-b \otimes a \otimes c+c \otimes a \otimes b-c \otimes b \otimes a \\
& v: a \otimes b \otimes c \mapsto a \otimes b c, \quad a, b, c \in A .
\end{aligned}
$$


Since the expressions $u(a \wedge b \otimes c)$ generate $\mathscr{L}^{3}(A)$, the long exact sequence (7-1) decomposes as a pair of short exact sequences

$$
\begin{aligned}
& 0 \rightarrow \Lambda^{3}(A) \rightarrow \Lambda^{2}(A) \otimes A \rightarrow \mathscr{L}^{3}(A) \rightarrow 0 \\
& 0 \rightarrow \mathscr{L}^{3}(A) \stackrel{p_{3}}{\rightarrow} A \otimes \mathrm{SP}^{2}(A) \rightarrow \mathrm{SP}^{3}(A) \rightarrow 0 .
\end{aligned}
$$

In particular the map

$$
\begin{gathered}
\mathscr{L}^{3}(A) \stackrel{p_{3}}{\longrightarrow} J^{3}(A) \\
{[a, b, c] \longmapsto(a, b, c)}
\end{gathered}
$$

induced by the projection $p_{3}(2-8)$ is an isomorphism.

Remark 7.1 (i) The sequences (7-4) and (7-5) both remain exact for an arbitrary group $A$. This is due to the fact that (7-5) derives for any $A$ to a long exact sequence for which the arrow

$$
\pi_{1}\left(A \stackrel{L}{\otimes} L \mathrm{SP}^{2} A\right) \longrightarrow L_{1} \mathrm{SP}^{3} A
$$

is surjective (as follows from the presentation (2-20) of $L_{1} \mathrm{SP}^{3} A$ ).

(ii) There is a natural isomorphism

$$
\mathscr{L}^{3}(A) \simeq E^{3}(A):=\operatorname{ker}\left\{\Gamma_{2}(A) \otimes A \rightarrow \Gamma_{3}(A)\right\} .
$$

This is obtained from the following prolongation of part of diagram (3-2) for $n=3$ :

$(7-8)$

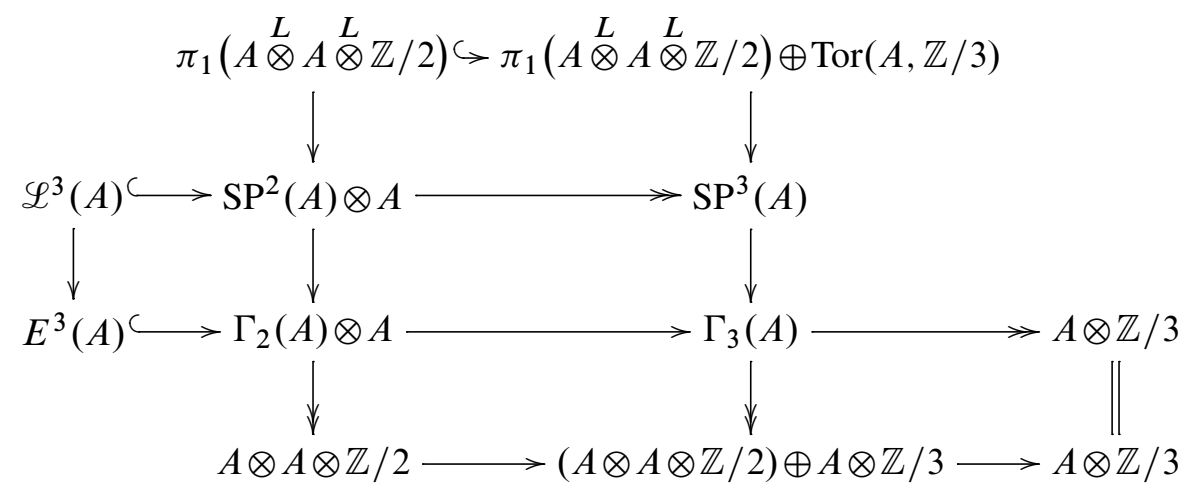

\subsection{The Curtis decomposition}

We will now consider higher Lie functors. Curtis gave in [14] a decomposition of the functors $\mathscr{L}^{n}(A)$ into functors $\mathrm{SP}^{n}, J^{n}$ and their iterates (see also Mikhailov and 
Passi [40]). For example, when $A$ is a free abelian group we have the following decompositions in low degrees:

$$
\begin{aligned}
\mathscr{L}^{1}(A)= & A \\
\mathscr{L}^{2}(A)= & J^{2}(A) \\
\mathscr{L}^{3}(A)= & J^{3}(A) \\
\mathscr{L}^{4}(A)= & J^{2}\left(J^{2}(A)\right) \oplus J^{4}(A) \\
\mathscr{L}^{5}(A)= & \left(J^{3}(A) \otimes J^{2}(A)\right) \oplus J^{5}(A) \\
\mathscr{L}^{6}(A)= & J^{3}\left(J^{2}(A)\right) \oplus J^{2}\left(J^{3}(A)\right) \oplus\left(J^{4}(A) \otimes J^{2}(A)\right) \oplus J^{6}(A) \\
\mathscr{L}^{7}(A)= & \left(J^{3}(A) \otimes \mathrm{SP}^{2}\left(J^{2}(A)\right)\right) \oplus\left(J^{5}(A) \otimes J^{2}(A)\right) \\
& \oplus\left(J^{2}\left(J^{2}(A)\right) \otimes J^{3}(A)\right) \oplus\left(J^{4}(A) \otimes J^{3}(A)\right) \oplus J^{7}(A) \\
\mathscr{L}^{8}(A)= & J^{2}\left(J^{2}\left(J^{2}(A)\right)\right) \oplus J^{2} J^{4} \oplus\left(J^{3}(A) \otimes J^{2}(A) \otimes J^{3}(A)\right) \\
& \oplus J^{5}(A) \otimes J^{3}(A) \oplus J^{4}\left(J^{2}(A)\right) \oplus\left(J^{4}(A) \otimes \mathrm{SP}^{2}\left(J^{2}(A)\right)\right) \\
& \oplus\left(J^{6}(A) \otimes J^{2}(A)\right) \oplus J^{8}(A)
\end{aligned}
$$

We will refer to these descriptions of the Lie functors as their Curtis decompositions. It should be understood that the splittings into direct sums displayed here are not functorial, and that all that exists functorially are filtrations of the $\mathscr{L}^{n}(A)$, whose associated graded components are the expressions displayed. As a matter of convenience, we will nevertheless refer to these expressions as summands of the Lie functors. We have already come across the cases $n=2,3$ of these decompositions in Proposition 2.1). The next two cases are the short exact sequences

$$
\begin{aligned}
& 0 \rightarrow \Lambda^{2} \Lambda^{2}(A) \rightarrow \mathscr{L}^{4}(A) \stackrel{p_{4}}{\rightarrow} J^{4}(A) \rightarrow 0 \\
& 0 \rightarrow \Lambda^{2}(A) \otimes J^{3}(A) \rightarrow \mathscr{L}^{5}(A) \stackrel{p_{5}}{\rightarrow} J^{5}(A) \rightarrow 0,
\end{aligned}
$$

where the left-hand arrows are respectively defined by

$$
\begin{aligned}
(a \wedge b) \wedge(c \wedge d) & \mapsto[[a, b],[c, d]] \\
(a \wedge b) \otimes(c, d, e) & \mapsto[[a, b],[c, d, e]] .
\end{aligned}
$$

It is a general fact that the final term in the decomposition of $\mathscr{L}^{n}(A)$ is always $J^{n}(A)$, the projection of $\mathscr{L}^{n}(A)$ onto $J^{n}(A)$ being the map $p_{n}(2-8)$. 


\subsection{Super-Lie functors}

We will now define super-Lie functors

$$
\mathscr{L}_{s}^{n}: \mathrm{Ab} \rightarrow \mathrm{Ab}, \quad n \geq 1
$$

Definition 7.2 (Leibowitz [33]) A graded Lie ring with squares (GLRS for short) is a graded abelian group $B=\bigoplus_{i=0}^{\infty} B_{i}$ with homomorphisms

$$
\{,\}: B_{i} \otimes B_{j} \rightarrow B_{i+j},
$$

[2]: $B_{n} \rightarrow B_{2 n}$ for $n$ odd

such that the following conditions are satisfied (for elements $x \in B_{i}, y \in B_{j}, z \in B_{k}$ ):

(1) $\{x, y\}+(-1)^{i j}\{y, x\}=0$

(2) $\{x, x\}=0$ for $i$ even

(3) $(-1)^{i k}\{\{x, y\}, z\}+(-1)^{j i}\{\{y, z\}, x\}+(-1)^{k j}\{\{z, x\}, y\}=0$

(4) $\{x, x, x\}=0$

(5) $(a x)^{[2]}=a^{2} x^{[2]}$ for $i$ odd, $a \in \mathbb{Z}$

(6) $(x+y)^{[2]}=x^{[2]}+y^{[2]}+\{x, y\}$ for $i=j$ odd

$$
\text { (7) }\left\{y, x^{[2]}\right\}=\{y, x, x\} \text { for } i \text { odd. }
$$

For an abelian group $A$, define $\mathscr{L}_{S}(A)$ to be the graded Lie ring with squares freely generated by $A$ in degree 1 . It may be defined as a GLRS together with a homomorphism of abelian groups $l: A \rightarrow \mathscr{L}_{S}(A)$ such that for every map $f: A \rightarrow B$ with $B$ a GLRS, there is a unique morphism of GLRS $d: \mathscr{L}_{S}(A) \rightarrow B$ such that $f=d \circ l$. The abelian group $\mathscr{L}_{S}(A)$ is naturally graded by $\mathscr{L}_{S}(A)=\bigoplus_{n=1}^{\infty} \mathscr{L}_{s}^{n}(A)$ and for any $x \in \mathscr{L}_{S}(A)$, we set $|x|=n$ whenever $x \in \mathscr{L}_{s}^{n}(A)$. The $n$-th graded piece $\mathscr{L}_{s}^{n}(A)$ is called the $n$-th super-Lie functor applied to $A$. In particular, there is a natural isomorphism

$$
\begin{gathered}
\Gamma_{2}(A) \simeq \mathscr{L}_{S}^{2}(A) \\
\gamma_{2}(a) \mapsto a^{[2]}
\end{gathered}
$$

analogous to the first isomorphism (2-11).

For any free abelian group $A$, a natural monomorphism

$$
z_{n}: \mathscr{L}_{s}^{n}(A) \rightarrow \otimes^{n}(A)
$$


is defined inductively on homogeneous elements by setting

$$
\begin{aligned}
\{x, y\} & \mapsto z_{|x|}(x) \otimes z_{|y|}(y)-(-1)^{|x||y|} z_{|y|}(y) \otimes z_{|x|}(x), \\
x^{[2]} & \mapsto z_{|x|}(x) \otimes z_{|x|}(x) .
\end{aligned}
$$

\subsection{The third super-Lie functor}

We will now adapt the discussion of Section 7.1 to the context of the super-Lie functors. The relations (7-16) and (7-17) imply that the group $\mathscr{L}_{S}^{3}(A)$ can be identified with the subgroup of $A \otimes A \otimes A$ generated by the elements

$$
a \otimes b \otimes c+b \otimes a \otimes c-c \otimes a \otimes b-c \otimes b \otimes a, \quad a, b, c \in A,
$$

which are the super analogues of the generators (2-2) of $\mathscr{L}^{3}(A)$. Let us now show that there is a natural isomorphism

$$
\begin{aligned}
\mathscr{L}_{s}^{3}(A) & \simeq Y^{3}(A) \\
\{\{a, b\}, c\} & \mapsto a \otimes b \wedge c+b \otimes a \wedge c, \quad a, b, c \in A .
\end{aligned}
$$

Consider, for any free abelian group $A$, the Koszul resolution $\operatorname{Kos}^{3}(A)(2-30)$ :

$$
0 \rightarrow \Gamma_{3}(A) \stackrel{i}{\rightarrow} \Gamma_{2}(A) \otimes A \stackrel{\bar{f}}{\rightarrow} A \otimes \Lambda^{2}(A) \rightarrow \Lambda^{3}(A) \rightarrow 0,
$$

where the maps $\bar{f}$ and $i$ are defined by

$$
\begin{array}{cl}
\bar{f}: \gamma_{2}(a) \otimes b \mapsto a \otimes a \wedge b, & a, b \in A, \\
i: \begin{cases}\gamma_{3}(a) \mapsto \gamma_{2}(a) \otimes a & a \in A, \\
\gamma_{2}(a) b \mapsto \gamma_{2}(a) \otimes b+a b \otimes a & a, b \in A .\end{cases}
\end{array}
$$

The map $\bar{f}$ factors as

$$
\Gamma_{2}(A) \otimes A \stackrel{\bar{u}}{\longrightarrow} A \otimes A \otimes A \stackrel{\bar{v}}{\longrightarrow} A \otimes \Lambda^{2}(A)
$$

where

$$
\begin{aligned}
& \bar{u}: \gamma_{2}(a) \otimes b \mapsto a \otimes a \otimes b-b \otimes a \otimes a, \\
& \bar{v}: a \otimes b \otimes c \mapsto a \otimes b \wedge c, \quad a, b, c \in A .
\end{aligned}
$$

It follows that

$$
\bar{u}(a b \otimes c)=\{\{a, b\}, c\}, \quad a, b, c \in A
$$

so that (7-20) decomposes as a pair of short exact sequences

$$
\begin{gathered}
0 \rightarrow \Gamma_{3}(A) \rightarrow \Gamma_{2}(A) \otimes A \rightarrow \mathscr{L}_{S}^{3}(A) \rightarrow 0 \\
0 \rightarrow \mathscr{L}_{S}^{3}(A) \rightarrow A \otimes \Lambda^{2}(A) \rightarrow \Lambda^{3}(A) \rightarrow 0
\end{gathered}
$$


similar to (7-4), (7-5). We see from the presentation (2-24) of $L_{1} \Lambda^{3} A$ that these two sequences remain exact when $A$ is an arbitrary abelian group.

\subsection{Higher super-Lie functors}

There exists a decomposition of super-Lie functors analogous to the Curtis decomposition of Lie functors, which we will now describe in low degrees. We begin by defining inductively an extension of the Lie superbracket (7-13) to left-normalized $n$-fold brackets, by setting

$$
\left\{a_{1}, \ldots, a_{n}\right\}=\left\{\left\{a_{1}, \ldots, a_{n-1}\right\}, a_{n}\right\} .
$$

The relations (7-15), (7-16) and (7-17) imply that the group $\mathscr{L}_{s}^{n}(A)$ is generated by the elements $\left\{a_{1}, \ldots, a_{n}\right\}$ for all $a_{i} \in A$, together with elements $b^{[2]}$ for $b \in \mathscr{L}_{s}^{k}(A)$ (whenever $k$ odd and $n=2 k$ ).

For all $n \geq 2$ and abelian group $A$, a natural epimorphism $\bar{p}_{n}: \mathscr{L}_{s}^{n}(A) \rightarrow Y^{n}(A)$ is defined by

$$
\begin{aligned}
\left\{a_{1}, a_{2}, \ldots, a_{n}\right\} & \mapsto a_{1} \otimes a_{2} \wedge \cdots \wedge a_{n}+a_{2} \otimes a_{1} \wedge a_{3} \wedge \cdots \wedge a_{n} \\
b^{[2]} & \mapsto 0 .
\end{aligned}
$$

Proposition 7.3 For any free abelian group $A$, the sequence of abelian groups

$$
0 \rightarrow \Lambda^{2} \Gamma_{2}(A) \stackrel{j}{\rightarrow} \mathscr{L}_{s}^{4}(A) \stackrel{\bar{p}_{4}}{\rightarrow} A \otimes \Lambda^{3}(A) \rightarrow \Lambda^{4}(A) \rightarrow 0
$$

is exact, with $j$ and $\bar{p}_{4}$ respectively defined by

$$
\begin{aligned}
j: \gamma_{2}\left(a_{1}\right) \wedge \gamma_{2}\left(a_{2}\right) & \mapsto\left\{a_{1}, a_{2}, a_{1}, a_{2}\right\} \\
\bar{p}_{4}:\left\{a_{1}, a_{2}, a_{3}, a_{4}\right\} & \mapsto a_{1} \otimes a_{2} \wedge a_{3} \wedge a_{4}+a_{2} \otimes a_{1} \wedge a_{3} \wedge a_{4} .
\end{aligned}
$$

The relations (7-15) and (7-16) imply that

$$
\left\{a_{1}, a_{2}, a_{1}, a_{2}\right\}=-\left\{a_{2}, a_{1}, a_{2}, a_{1}\right\}, \quad a_{1}, a_{2} \in A .
$$

In addition

$$
j:\left(\gamma_{2}(a+b)-\gamma_{2}(a)-\gamma_{2}(b)\right) \wedge \gamma_{2}(c) \mapsto\{a, c, b, c\}-\{b, c, a, c\}, \quad a, b, c \in A,
$$

so that the map $j$ is well-defined.

Remark 7.4 For an arbitrary abelian group $A$, the isomorphism (2-6) no longer holds, and the exact sequence (7-25) is replaced by an exact sequence

$$
0 \rightarrow \Lambda^{2} Y^{2}(A) \stackrel{j}{\rightarrow} \mathscr{L}_{s}^{4}(A) \rightarrow A \otimes \Lambda^{3}(A) \rightarrow \Lambda^{4}(A) \rightarrow 0
$$


where $j\left(\left(a_{1} \otimes a_{1}\right) \wedge\left(a_{2} \otimes a_{2}\right)\right)=\left\{a_{1}, a_{2}, a_{1}, a_{2}\right\}$. The following long exact sequence describes the relation between the functors $\Gamma_{2}$ and $Y^{2}$ in this general case:

$$
0 \rightarrow R_{2}(A) \rightarrow \operatorname{Tor}(A, A) \rightarrow \Omega_{2}(A) \rightarrow \Gamma_{2}(A) \rightarrow Y^{2}(A) \rightarrow 0 .
$$

Let us define a functor $\tilde{Y}^{n}$ by the short exact sequence

$$
0 \longrightarrow \tilde{Y}^{n}(A) \stackrel{j}{\longrightarrow} \mathscr{L}_{s}^{n}(A) \stackrel{\bar{p}_{n}}{\longrightarrow} Y^{n}(A) \longrightarrow 0 .
$$

Sequence (7-27) asserts in particular that

$$
\tilde{Y}^{4}(A)=\Lambda^{2} Y^{2}(A),
$$

so we have the following superanalogue for $n=4$ of the short exact sequence (2-9):

$$
0 \longrightarrow \Lambda^{2} Y^{2}(A) \stackrel{j}{\longrightarrow} \mathscr{L}_{S}^{4}(A) \stackrel{\bar{p}_{4}}{\longrightarrow} Y^{4}(A) \longrightarrow 0 .
$$

Similarly, the short exact sequence (7-28) for $n=5$, which is the superanalogue of the decomposition (7-11) of $\mathscr{L}^{5}(A)$, is described more precisely by the short exact sequence

$$
0 \longrightarrow Y^{3}(A) \otimes \Gamma_{2}(A) \stackrel{h}{\longrightarrow} \mathscr{L}_{s}^{5}(A) \stackrel{\bar{p}_{5}}{\longrightarrow} Y^{5}(A) \longrightarrow 0,
$$

where the arrow $h$ is defined by

$$
h:\{a, b, c\} \otimes \gamma_{2}(d) \mapsto\{a, b, c, d, d\} .
$$

Remark 7.5 One can show that there is a natural filtration on the term $\tilde{Y}^{6}$, with an associated graded component $\Gamma_{2} Y^{3}(A)$, so that $\mathscr{L}_{S}^{6}(A)$ can have some 4-torsion whenever there is some 2-torsion in the group $A$. In fact, this is a general phenomenon: for all $k \geq 1$, there may be some 4 -torsion in $\mathscr{L}_{s}^{4 k+2}(A)$ whenever $A$ is a 2 -torsion group, whereas there will only be 2 -torsion in all other components of the super-Lie algebra $\mathscr{L}_{S}(A)$.

\subsection{Relations between Lie and super-Lie functors}

Let $A$ be a free abelian group and consider, for $n \geq 2$, the natural monomorphisms

$$
\begin{aligned}
& c_{n}: \mathscr{L}^{n}(A) \rightarrow \otimes^{n} A, \\
& z_{n}: \mathscr{L}_{s}^{n}(A) \rightarrow \otimes^{n} A .
\end{aligned}
$$


For $n=3$ we have by (7-3), (7-18), for $a, b, c \in A$ :

$$
\begin{gathered}
c_{3}:[a, b, c] \mapsto a \otimes b \otimes c-b \otimes a \otimes c-c \otimes a \otimes b+c \otimes b \otimes a \\
z_{3}:\{a, b, c\} \mapsto a \otimes b \otimes c+b \otimes a \otimes c-c \otimes a \otimes b-c \otimes b \otimes a .
\end{gathered}
$$

For any pair of free abelian groups $A$ and $B$, and $n \geq 2$, we define a pair of morphisms

$$
\begin{aligned}
& \chi_{n}: \mathscr{L}_{s}^{n}(A) \otimes \Lambda^{n}(B) \rightarrow \mathscr{L}^{n}(A \otimes B) \\
& \bar{\chi}_{n}: \mathscr{L}^{n}(A) \otimes \Lambda^{n}(B) \rightarrow \mathscr{L}_{s}^{n}(A \otimes B)
\end{aligned}
$$

for $a_{1}, \ldots, a_{n} \in A$ and $b_{1}, \ldots, b_{n} \in B$, by

$$
\begin{gathered}
\chi_{n}:\left\{a_{1}, \ldots, a_{n}\right\} \otimes b_{1} \wedge \cdots \wedge b_{n} \mapsto \sum_{\sigma \in \Sigma_{n}} \operatorname{sign}(\sigma)\left[a_{1} \otimes b_{\sigma_{1}}, \ldots, a_{n} \otimes b_{\sigma_{n}}\right] \\
\bar{\chi}_{n}:\left[a_{1}, \ldots, a_{n}\right] \otimes b_{1} \wedge \cdots \wedge b_{n} \mapsto \sum_{\sigma \in \Sigma_{n}} \operatorname{sign}(\sigma)\left\{a_{1} \otimes b_{\sigma_{1}}, \ldots, a_{n} \otimes b_{\sigma_{n}}\right\} .
\end{gathered}
$$

For this definition of $\chi_{n}$ to be complete, we also need the following rule for $\chi_{n}$ whenever $n=2 k$ with $k$ odd:

$$
\begin{aligned}
\left\{a_{1}, \ldots, a_{k}\right\}^{[2]} \otimes b_{1} \wedge & \cdots \\
& \mapsto b_{n} \\
& \left.\mapsto\left[a_{\sigma \in A_{n}} \otimes b_{\sigma_{1}}, \ldots, a_{k} \otimes b_{\sigma_{k}}\right],\left[a_{1} \otimes b_{\sigma_{k+1}}, \ldots, a_{k} \otimes b_{\sigma_{2 k}}\right]\right] .
\end{aligned}
$$

Theorem 7.6 Let $A$ and $B$ be free abelian groups. The following diagrams with arrows defined by (7-32), (7-33), (2-14) are commutative:
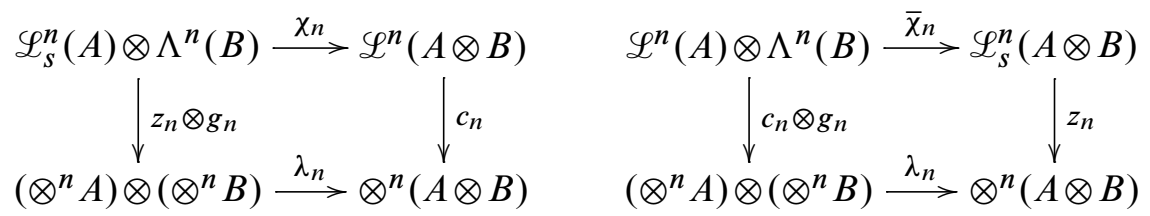

Proof Let us begin by considering the first diagram (7-34) for $n=2$. The commutativity of diagram

$$
\begin{array}{cc}
\Gamma_{2}(A) \otimes \Lambda^{2}(B) \stackrel{\chi_{2}}{\longrightarrow} & \Lambda^{2}(A \otimes B) \\
\downarrow^{z_{2} \otimes g_{2}} & \\
(A \otimes A) \otimes(B \otimes B) & \stackrel{\lambda_{2}}{\longrightarrow}(A \otimes B) \otimes(A \otimes B)
\end{array}
$$

can be checked directly: for any $a_{1}, \in A, b_{1}, b_{2} \in B$ :

$$
\begin{aligned}
\lambda_{2} \circ\left(z_{2} \otimes g_{2}\right)\left(\gamma_{2}\left(a_{1}\right) \otimes b_{1} \wedge b_{2}\right) & =c_{2} \circ \chi_{2}\left(\gamma_{2}\left(a_{1}\right) \otimes b_{1} \wedge b_{2}\right) \\
& =\left(a_{1} \otimes b_{1}\right) \otimes\left(a_{1} \otimes b_{2}\right)-\left(a_{1} \otimes b_{2}\right) \otimes\left(a_{1} \otimes b_{1}\right) .
\end{aligned}
$$


By induction on $n$, we find that

$z_{n} \otimes g_{n}\left(\left\{a_{1}, \ldots, a_{n}\right\} \otimes b_{1} \wedge \cdots \wedge b_{n}\right)$

Hence

$$
\begin{aligned}
= & \sum_{\sigma \in \Sigma_{n}} \operatorname{sign}(\sigma) z_{n}\left\{a_{1}, \ldots, a_{n}\right\} \otimes b_{\sigma_{1}} \otimes \cdots \otimes b_{\sigma_{n}} \\
= & \sum_{\sigma \in \Sigma_{n}} \operatorname{sign}(\sigma) z_{n-1}\left\{a_{1}, \ldots, a_{n-1}\right\} \otimes a_{n} \otimes b_{\sigma_{1}} \otimes \cdots \otimes b_{\sigma_{n}} \\
& +(-1)^{n} \sum_{\sigma \in \Sigma_{n}} \operatorname{sign}(\sigma) a_{n} \otimes z_{n-1}\left\{a_{1}, \ldots, a_{n-1}\right\} \otimes b_{\sigma_{1}} \otimes \cdots \otimes b_{\sigma_{n}} .
\end{aligned}
$$

$\lambda_{n} \circ\left(z_{n} \otimes g_{n}\right)\left(\left\{a_{1}, \ldots, a_{n}\right\} \otimes b_{1} \wedge \cdots \wedge b_{n}\right)$

$$
\begin{gathered}
=\sum_{j=1}^{n} \lambda_{n-1}\left(\sum_{\substack{\eta=\left\{\sigma_{1}, \ldots, \sigma_{n-1}\right\} \\
\in\{1, \ldots, \hat{j}, \ldots, n\}}} \operatorname{sign}(\eta, j) z_{n-1}\left\{a_{1}, \ldots, a_{n-1}\right\} \otimes b_{\sigma_{1}} \otimes \cdots \otimes b_{\sigma_{n-1}}\right) \otimes\left(a_{n} \otimes b_{j}\right) \\
+(-1)^{n} \sum_{j=1}^{n}\left(a_{n} \otimes b_{j}\right) \\
\otimes \lambda_{n-1}\left(\sum_{\substack{\eta=\left\{\sigma_{1}, \ldots, \sigma_{n-1}\right\} \\
\in\{1, \ldots, \hat{j}, \ldots, n\}}} \operatorname{sign}(j, \eta) z_{n-1}\left\{a_{1}, \ldots, a_{n-1}\right\} \otimes b_{\sigma_{1}} \otimes \cdots \otimes b_{\sigma_{n-1}}\right) \\
=\sum_{j=1}^{n}\left(\sum_{\substack{\eta=\left\{\sigma_{1}, \ldots, \sigma_{n-1}\right\} \\
\epsilon\{1, \ldots, \hat{j}, \ldots, n\}}}^{\operatorname{sign}(\eta, j)\left(c_{n-1}\left[a_{1} \otimes b_{\sigma_{1}}, \ldots, a_{n-1} \otimes b_{\sigma_{n-1}}\right] \otimes\left(a_{n} \otimes b_{j}\right)\right.}\right) \\
\left.\left.-\left(a_{n} \otimes b_{j}\right) \otimes c_{n-1}\left[a_{1} \otimes b_{\sigma_{1}}, \ldots, a_{n-1} \otimes b_{\sigma_{n-1}}\right]\right)\right)
\end{gathered}
$$$$
=\sum_{\sigma \in \Sigma_{n}} \operatorname{sign}(\sigma) c_{n}\left[a_{1} \otimes b_{\sigma_{1}}, \ldots, a_{n} \otimes b_{\sigma_{n}}\right]=c_{n} \circ \chi_{n}\left(\left\{a_{1}, \ldots, a_{n}\right\} \otimes b_{1} \wedge \cdots \wedge b_{n}\right) .
$$

One can prove in a similar manner that

$$
\begin{aligned}
c_{2 k} \circ \chi_{2 k}\left(\left\{a_{1}, \ldots, a_{k}\right\}^{[2]}\right. & \left.\otimes b_{1} \wedge \cdots \wedge b_{2 k}\right) \\
& =\lambda_{2 k} \circ\left(z_{2 k} \otimes g_{2 k}\right)\left(\left\{a_{1}, \ldots, a_{k}\right\}^{[2]} \otimes b_{1} \wedge \cdots \wedge b_{2 k}\right) .
\end{aligned}
$$

for an odd $k$, so that the commutativity of the first diagram (7-34) is proved. The commutativity of the second diagram is proved in the same way: for $n=2$, it follows from the commutativity of the diagram (2-33) and one then simply repeats the previous computation for a general $n$, with appropriate changes in the signs of the various expressions. 
For any pair of abelian groups $A, B$, we define a natural arrow:

$$
\beta_{n}: Y^{n}(A) \otimes \Lambda^{n}(B) \rightarrow J^{n}(A \otimes B)
$$

$$
\begin{array}{r}
\beta_{n}: \bar{p}_{n}\left\{a_{1}, \ldots, a_{n}\right\} \otimes b_{1} \wedge \cdots \wedge b_{n} \mapsto \sum_{\sigma \in \Sigma_{n}} \operatorname{sign}(\sigma) p_{n}\left[a_{1} \otimes b_{\sigma_{1}}, \ldots, a_{n} \otimes b_{\sigma_{n}}\right], \\
a_{1}, \ldots, a_{n} \in A, b_{1}, \ldots, b_{n} \in B .
\end{array}
$$

Proposition 7.7 The following diagram with exact columns is commutative:

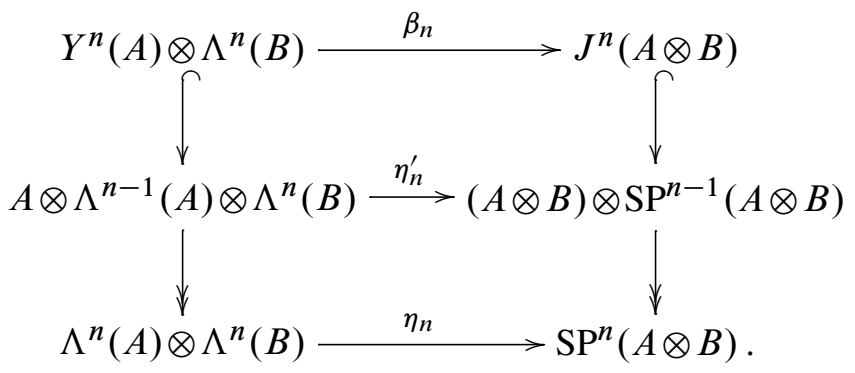

Here the map $\eta_{n}$ is defined by (2-34) and $\eta_{n}^{\prime}$ is by $\eta_{n}^{\prime}: a_{1} \otimes a_{2} \wedge \cdots \wedge a_{n} \otimes b_{1} \wedge \cdots \wedge b_{n}$

$$
\mapsto \sum_{\sigma \in \Sigma_{n}} \operatorname{sign}(\sigma)\left(a_{1} \otimes b_{\sigma_{1}}\right) \otimes\left(a_{2} \otimes b_{\sigma_{2}}\right) \ldots\left(a_{n} \otimes b_{\sigma_{n}}\right) .
$$

The proof of this proposition follows directly from the definition of the various maps.

\section{Derived functors of Lie functors}

It is asserted in Schlesinger [45] that if $p$ is an odd prime then the groups $L_{n+k} \mathscr{L}^{p}(\mathbb{Z}, n)$ are $p$-torsion for all $k$, and in particular

$$
L_{n+k} \mathscr{L}^{p}(\mathbb{Z}, n)= \begin{cases}\mathbb{Z} / p & k=2 i(p-1)-1, i=1,2, \ldots,[n / 2], \\ 0 & \text { otherwise. }\end{cases}
$$

In the next three subsections, we will give a direct proof of this fact for $p=3$, in other words show that

$$
L_{n+k} \mathscr{L}^{3}(\mathbb{Z}, n)= \begin{cases}\mathbb{Z} / 3 & k=4 i-1, i=1,2, \ldots,[n / 2], \\ 0 & \text { otherwise, }\end{cases}
$$

and we will more generally compute in Theorem 8.2 the derived functors $L_{n+k} \mathscr{L}^{3}(A, n)$ for a general abelian group $A$. Note that the derived functors of the Lie functors $\mathscr{L}^{q}$ 
are complicated when $q$ a composite number. We refer to Mikhailov and Passi [40, page 280] for a description of these derived functors in low degrees. One finds for example that

$$
L_{i} \mathscr{L}^{8}(\mathbb{Z}, 1)= \begin{cases}\mathbb{Z} / 2 & i=4,5,7, \\ 0 & i \neq 4,5,7 .\end{cases}
$$

The gap in the homotopy groups which occurs here for $i=6$ is the illustration of a general phenomenon which, as we will see for example in (8-16), also occurs in more elaborate contexts.

Returning to the $p=3$ case, let us first observe that for any abelian group $A$ the exact sequence (7-5) derives to a long exact sequence:

$$
\begin{aligned}
& \rightarrow L_{i+1} \mathscr{L}^{3}(A, n) \rightarrow \pi_{i+1}\left(A[n] \otimes L \mathrm{SP}^{2}(A, n)\right) \rightarrow L_{i+1} \mathrm{SP}^{3}(A, n) \\
& \rightarrow L_{i} \mathscr{L}^{3}(A, n) \rightarrow \pi_{i}\left(A[n] \otimes L \operatorname{SP}^{2}(A, n)\right) \rightarrow L_{i} \operatorname{SP}^{3}(A, n) \rightarrow
\end{aligned}
$$

In addition, the isomorphism (2-42) implies that

$$
L_{i} Y^{3}(A, n) \simeq L_{i+3} \mathscr{L}^{3}(A, n+1), \quad \text { for all } i \geq 3 .
$$

\subsection{The derived functors $L_{i} \mathscr{L}^{3}(A)$}

The Koszul sequence (2-32) associated for $n=3$ to a flat 2-term resolution $f: L \rightarrow M$ of an abelian group $A$ may be written as

$$
0 \rightarrow \mathscr{L}_{s}^{3}(L) \rightarrow L \otimes M \otimes L \stackrel{\delta}{\rightarrow} L \otimes M \otimes M \rightarrow \mathscr{L}^{3}(M) \rightarrow \mathscr{L}^{3}(A) \rightarrow 0
$$

where $\delta\left(l \otimes m \otimes l^{\prime}\right)=l \otimes m \otimes f\left(l^{\prime}\right)+l^{\prime} \otimes f(l) \otimes m-l \otimes f\left(l^{\prime}\right) \otimes m, \quad l, l^{\prime} \in L, m \in M$.

The three middle terms constitute a complex which represents the object $L \mathscr{L}^{3}(A)$ of the derived category. In particular, if one considers the resolution $\mathbb{Z} \stackrel{m}{\rightarrow} \mathbb{Z}$ of $\mathbb{Z} / m$, one finds that

$$
L_{i} \mathscr{L}^{3}(\mathbb{Z} / m)= \begin{cases}\mathbb{Z} / m & i=1, \\ 0 & i \neq 1 .\end{cases}
$$

By (2-20), the natural transformation $\operatorname{Tor}\left(\mathscr{Y}_{2}(A), A\right) \rightarrow \mathscr{Y}_{3}(A)$ is an epimorphism. More generally, the exact sequence (8-3) provides the following description of the derived functors of $\mathscr{L}^{3}$ :

$$
\begin{gathered}
L_{2} \mathscr{L}^{3}(A)=\operatorname{ker}\left\{\operatorname{Tor}\left(\mathscr{Y}_{2}(A), A\right) \rightarrow \mathscr{Y}_{3}(A)\right\} \\
0 \rightarrow \operatorname{ker}\left\{\mathscr{Y}_{2}(A) \otimes A \rightarrow L_{1} \operatorname{SP}^{3}(A)\right\} \rightarrow L_{1} \mathscr{L}^{3}(A) \rightarrow \operatorname{Tor}\left(\operatorname{SP}^{2}(A), A\right) \rightarrow 0 \\
L_{i} \mathscr{L}^{3}(A)=0, \quad i>2 .
\end{gathered}
$$




\subsection{The derived functors $L_{i} \mathscr{L}^{3}(A, 1)$}

The short exact sequence (7-22) derives to a long exact sequence

$$
\begin{aligned}
\cdots \rightarrow L_{i+1} \mathscr{L}_{s}^{3}(A, n) \rightarrow \pi_{i+1}\left(A[n] \stackrel{L}{\otimes} L \Lambda^{2}(A, n)\right) \rightarrow L_{i+1} \Lambda^{3}(A, n) \\
\rightarrow L_{i} \mathscr{L}_{s}^{3}(A, n) \rightarrow \pi_{i}\left(A[n] \stackrel{L}{\otimes} L \Lambda^{2}(A, n)\right) \rightarrow L_{i} \Lambda^{3}(A, n) \rightarrow \cdots
\end{aligned}
$$

analogous to (8-3). For $n=0$ this reduces to the exact sequence

$$
\begin{aligned}
& 0 \rightarrow L_{2} Y^{3}(A) \rightarrow \operatorname{Tor}\left(\Omega_{2}(A), A\right) \Omega_{3}(A) \\
& \qquad \rightarrow L_{1} Y^{3}(A) \rightarrow \pi_{1}\left(L \Lambda^{2}(A) \stackrel{L}{\otimes} A\right) \rightarrow L_{1} \Lambda^{3}(A) \rightarrow 0 .
\end{aligned}
$$

This exact sequence is consistent with the results in Breen [10, Proposition 6.15], and with the presentation (2-24) of the groups $L_{i} \Lambda^{n}(A)$. The latter implies that the composite arrow

$$
L_{1} \Lambda^{2}(A) \otimes A \rightarrow \pi_{1}\left(L \Lambda^{2}(A) \stackrel{L}{\otimes} A\right) \rightarrow L_{1} \Lambda^{3}(A)
$$

is an epimorphism. The Künneth formula, together with the exact sequence (8-6), determines a 3-step filtration of $L_{1} Y^{3}(A)$. Taking into account the isomorphism (8-4) for $n=0$, we obtain the following description of the derived functors $L_{i} \mathscr{L}^{3}(A, 1)$ :

$$
\begin{aligned}
L_{3} \mathscr{L}^{3}(A, 1) & \simeq Y^{3}(A) \\
\operatorname{gr}_{1} L_{4} \mathscr{L}^{3}(A, 1) & \simeq \operatorname{gr}_{1} L_{1} Y^{3}(A) \simeq \operatorname{coker}\left\{\operatorname{Tor}\left(\Omega_{2}(A), A\right) \rightarrow \Omega_{3}(A)\right\} \\
\operatorname{gr}_{2} L_{4} \mathscr{L}^{3}(A, 1) & \simeq \operatorname{gr}_{2} L_{1} Y^{3}(A) \simeq \operatorname{ker}\left\{\Omega_{2}(A) \otimes A \rightarrow L_{1} \Lambda^{3}(A)\right\} \\
\operatorname{gr}_{3} L_{4} \mathscr{L}^{3}(A, 1) & \simeq \operatorname{gr}_{3} L_{1} Y^{3}(A) \simeq \operatorname{Tor}\left(\Lambda^{2}(A), A\right) \\
& L_{5} \mathscr{L}^{3}(A, 1) \simeq L_{2} Y^{3}(A) \simeq \operatorname{ker}\left\{\operatorname{Tor}\left(\Omega_{2}(A), A\right) \rightarrow \Omega_{3}(A)\right\}
\end{aligned}
$$

Remark 8.1 The natural map

$$
\operatorname{Tor}\left(\Omega_{2}(A), A\right) \simeq \pi_{2}\left(L \Lambda^{2}(A) \stackrel{L}{\otimes} A\right) \rightarrow \Omega_{3}(A)
$$

in the exact sequence (8-6) is in general neither injective nor surjective. This can be seen by considering the generators $\omega_{i}^{h}(x)$ (2-23) of the groups $\Omega_{n}(A)$. We know by Breen $[10,5.14]$ that the diagram

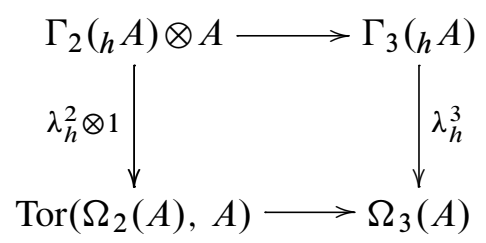


is commutative. It follows from the relation $\gamma_{2}(x) x=3 \gamma_{3}(x)$ in $\Gamma_{3}(A)$ that, with the notation introduced in (2-23), the corresponding relation

$$
\omega_{2}^{h}(x) * x=3 \omega_{3}^{h}(x)
$$

is satisfied in $\Omega_{3}(A)$ for all $x \in{ }_{h} A$. In particular, this implies that the arrow (8-9) is trivial for $h=3$ and $A=\mathbb{Z}_{3}$. Moreover, it is asserted in Baues and Buth [3] that

$$
L_{1} Y^{3}(\mathbb{Z} / 3)=\mathbb{Z} / 9 .
$$

We refer to Proposition A.1 in Appendix A for a proof by our methods of this assertion.

\subsection{The derived functors $L_{i} \mathscr{L}^{3}(A, 2)$}

The décalage isomorphisms (2-39) and Künneth formula yield a description of the groups

as follows:

$$
\pi_{r}\left(L \operatorname{SP}^{j}(A, 2) \stackrel{L}{\otimes} A[2]\right)
$$

$$
\begin{aligned}
& \pi_{2 j+2}\left(L \operatorname{SP}^{j}(A, 2) \stackrel{L}{\otimes} A[2]\right) \simeq \Gamma_{j}(A) \otimes A \\
& 0 \rightarrow L_{i-2} \Gamma_{j}(A) \otimes A \rightarrow \pi_{2 j+i}\left(L \operatorname{SP}^{j}(A, 2) \stackrel{L}{\otimes} A[2]\right) \rightarrow \operatorname{Tor}\left(L_{i-3} \Gamma_{j}(A), A\right) \rightarrow 0, \\
& i=3, \ldots, j+1, \\
&\left.\pi_{3 j+2}\left(L \operatorname{SP}^{j}(A, 2) \stackrel{L}{\otimes} A[2]\right)\right) \simeq \operatorname{Tor}\left(L_{j-1} \Gamma_{j}(A), A\right)
\end{aligned}
$$

From (2-26) and (5-2) we have the following commutative diagram, a prolongation of $(7-8)$ :

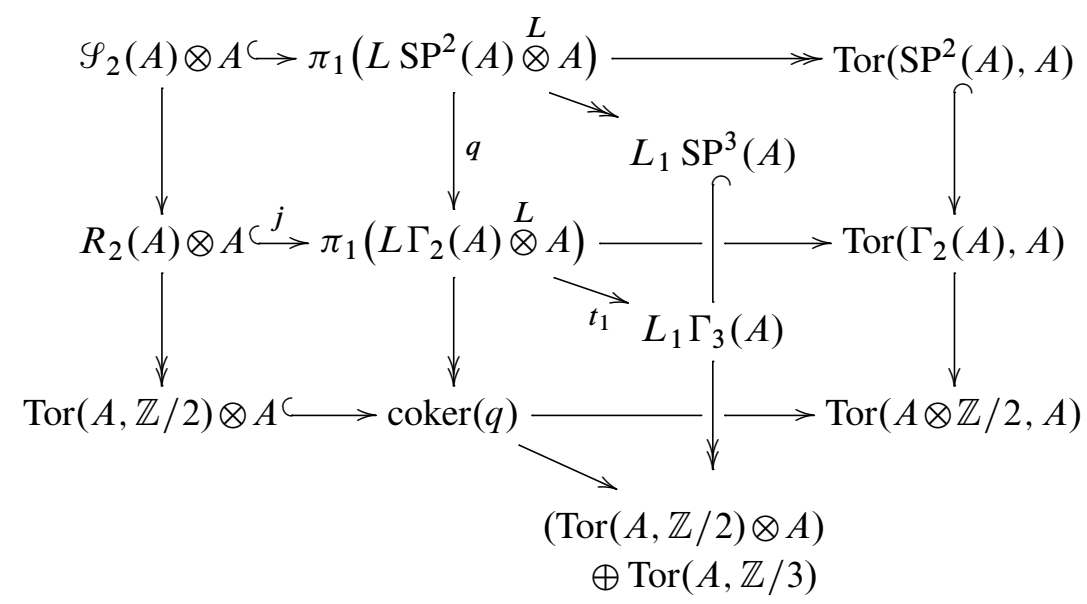


A diagram chase yields a canonical isomorphism $\operatorname{coker}\left(t_{1}\right) \simeq \operatorname{Tor}(A, \mathbb{Z} / 3)$. We obtain the following description of a portion of the long exact sequence (8-3) for $n=2$, where $t_{2}:=t_{1} \circ j$ :

$$
R_{2}(A) \otimes A \stackrel{t_{2}}{\longrightarrow} L_{1} \Gamma_{3}(A) \longrightarrow L_{6} \mathscr{L}^{3}(A, 2)
$$$$
\longrightarrow \Gamma_{2}(A) \otimes A \longrightarrow \Gamma_{3}(A) \rightarrow A \otimes \mathbb{Z} / 3
$$

From this we may extract by (7-7) the upper short exact sequence in the following commutative diagram, in which the vertical arrows are suspension maps:

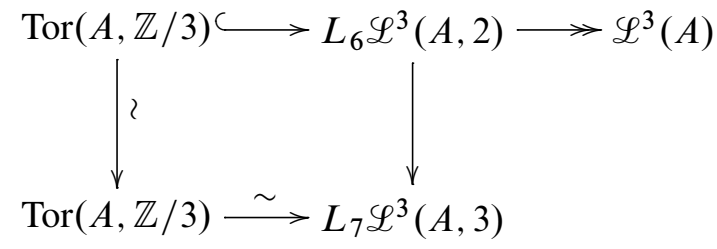

(we refer to Theorem 8.2 for this description of $L_{7} \mathscr{L}^{3}(A, 3)$ ). This determines a functorial direct sum decomposition of $L_{6} \mathscr{L}^{3}(A, 2)=L_{3} Y^{3}(A, 1)$. In addition, we may prolong diagram (8-13) by the following exact sequence:

$$
0 \rightarrow L_{8} \mathscr{L}^{3}(A, 2) \rightarrow \operatorname{Tor}\left(R_{2}(A), A\right) \rightarrow R_{3}(A) \rightarrow L_{7} \mathscr{L}^{3}(A, 2) \rightarrow \operatorname{ker}\left(t_{2}\right) \rightarrow 0 .
$$

A diagram chase in the following diagram

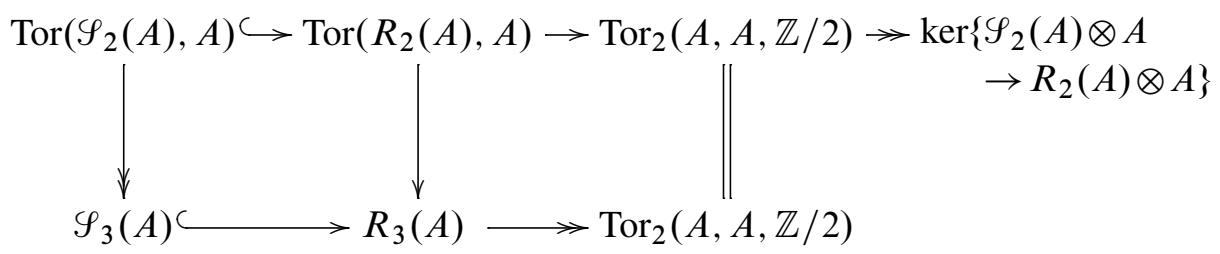

(in which we observe that the left-hand vertical arrow is surjective) then implies the following two equalities:

$$
\begin{aligned}
\operatorname{ker}\left\{\operatorname{Tor}\left(\mathscr{S}_{2}(A), A\right) \rightarrow \mathscr{S}_{3}(A)\right\} & =\operatorname{ker}\left\{\operatorname{Tor}\left(R_{2}(A), A\right) \rightarrow R_{3}(A)\right\} \\
\operatorname{coker}\left\{\operatorname{Tor}\left(R_{2}(A), A\right) \rightarrow R_{3}(A)\right\} & =\operatorname{ker}\left\{\mathscr{Y}_{2}(A) \otimes A \rightarrow R_{2}(A) \otimes A\right\} .
\end{aligned}
$$


Taking once more into account the décalage isomorphisms (8-4), this provides a complete description of the functors $L_{i} \mathscr{L}^{3}(A, 2)$ :

$$
\begin{aligned}
L_{5} \mathscr{L}^{3}(A, 2) & =L_{2} Y^{3}(A, 1)=A \otimes \mathbb{Z} / 3 \\
L_{6} \mathscr{L}^{3}(A, 2) & =L_{3} Y^{3}(A, 1)=\mathscr{L}^{3}(A) \oplus \operatorname{Tor}(A, \mathbb{Z} / 3) \\
\operatorname{gr}_{1} L_{7} \mathscr{L}^{3}(A, 2) & =\operatorname{gr}_{1} L_{4} Y^{3}(A, 1)=\operatorname{ker}\left\{\varphi_{2}(A) \otimes A \rightarrow R_{2}(A) \otimes A\right\} \\
\operatorname{gr}_{2} L_{7} \mathscr{L}^{3}(A, 2) & =\operatorname{gr}_{2} L_{4} Y^{3}(A, 1)=\operatorname{ker}\left\{R_{2}(A) \otimes A \rightarrow L_{1} \Gamma_{3}(A)\right\} \\
\operatorname{gr}_{3} L_{7} \mathscr{L}^{3}(A, 2) & =\operatorname{gr}_{3} L_{4} Y^{3}(A, 1)=\operatorname{Tor}\left(\Gamma_{2}(A), A\right) \\
L_{8} \mathscr{L}^{3}(A, 2) & =L_{5} Y^{3}(A, 1)=L_{2} \mathscr{L}^{3}(A) .
\end{aligned}
$$

For all other values of $i, L_{i} \mathscr{L}^{3}(A, 2)=0$.

As an illustration of these results, we will now give an explicit description of the isomorphism

$$
A \otimes \mathbb{Z} / 3 \stackrel{\sim}{\rightarrow} L_{2} Y^{3}(A, 1) \quad\left(=L_{2} \mathscr{L}_{S}^{3}(A, 1)\right)
$$

occurring in the first equation of (8-14), even though this will not be used in the sequel. Consider the simplicial model (2-17) of $L \mathscr{L}_{S}^{3}(A, 1)$ associated to a free resolution (2-15) of $A$. The isomorphism (8-15) is induced by the map

$$
A \otimes \mathbb{Z} / 3 \rightarrow \mathscr{L}_{s}^{3}\left(L \oplus s_{1}(M) \oplus s_{0}(L)\right) / \partial_{0}\left(\bigcap_{i=1}^{3} \partial_{i}\right)
$$

defined, for a chosen lifting $a$ to $M$ of $\bar{a} \in A \otimes \mathbb{Z} / 3$, by

$$
\bar{a} \mapsto\left\{s_{1}(a), s_{0}(a), s_{1}(a)\right\} .
$$

In order for this map to be well-defined, we must verify that

$$
3\left\{s_{1}(a), s_{0}(a), s_{1}(a)\right\} \in \partial_{0}\left(\operatorname{ker}\left(\partial_{1}\right) \cap \operatorname{ker}\left(\partial_{2}\right) \cap \operatorname{ker}\left(\partial_{3}\right)\right) .
$$

This is true since the element

$$
\begin{aligned}
& \eta:=3\left\{s_{2} s_{0}(a), s_{1} s_{0}(a), s_{2} s_{0}(a)\right\}-\left\{s_{2} s_{1}(a), s_{1} s_{0}(a), s_{2} s_{0}(a)\right\} \\
&+\left\{s_{2} s_{1}(a), s_{2} s_{0}(a), s_{1} s_{0}(a)\right\} \\
& \in \mathscr{L}_{s}^{3}\left(s_{0}\left(A_{1}\right) \oplus s_{1}\left(A_{1}\right) \oplus s_{2}\left(A_{1}\right) \oplus s_{1} s_{0}\left(A_{0}\right) \oplus s_{2} s_{0}\left(A_{0}\right) \oplus s_{2} s_{1}\left(A_{0}\right)\right)
\end{aligned}
$$

satisfies the equations $\partial_{i}(\eta)=0, i=1,2,3$ and $\partial_{0}(\eta)=3\left\{s_{1}(a), s_{0}(a), s_{1}(a)\right\}$. 


\subsection{The derived functors $L_{i} \mathscr{L}^{3}(A, n)$ for $n \geq 3$}

In each of the following three commutative diagrams, the exactness of the upper short exact sequence follows from Proposition 4.1 and the exactness of the lower one from Theorem 5.2. For $n \geq 3$ odd:

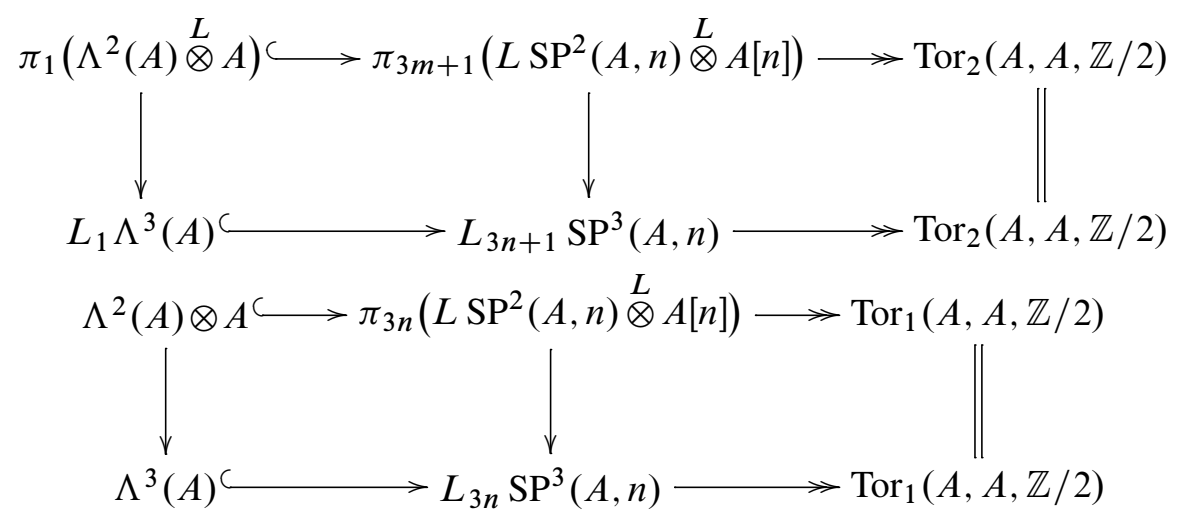

and for $n>3$ even:

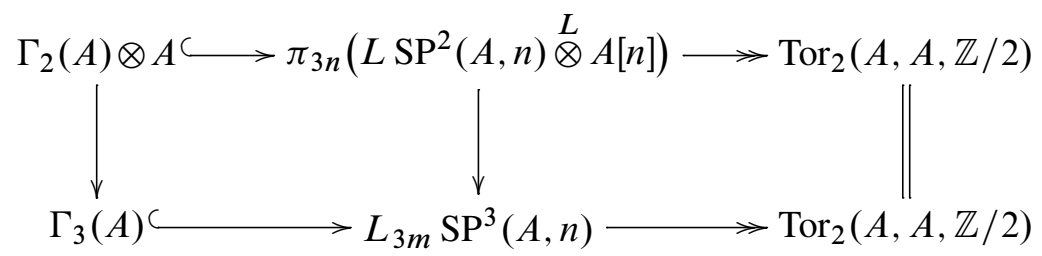

The sequence (8-3) therefore determines the following exact sequences:

Case I $n$ is odd $\geq 3$.

$$
\begin{aligned}
0 \rightarrow L_{3 n+2} \mathscr{L}^{3}(A, n) \rightarrow & \operatorname{Tor}\left(\Omega_{2}(A), A\right) \rightarrow \Omega_{3}(A) \\
\rightarrow L_{3 n+1} \mathscr{L}^{3}(A, n) \rightarrow \pi_{1}\left(L \Lambda^{2}(A) \otimes A\right) & \rightarrow L_{1} \Lambda^{3}(A) \\
\rightarrow L_{3 n} \mathscr{L}^{3}(A, n) \rightarrow \Lambda^{2}(A) \otimes A & \rightarrow \Lambda^{3}(A) \rightarrow L_{3 n-1} \mathscr{L}^{3}(A, n)
\end{aligned}
$$

Case II $n$ is even.

$$
\begin{aligned}
0 \rightarrow L_{3 n+2} \mathscr{L}^{3}(A, n) \rightarrow \operatorname{Tor}\left(R_{2}(A), A\right) \rightarrow R_{3}(A) & \\
\rightarrow L_{3 n+1} \mathscr{L}^{3}(A, n) \rightarrow \pi_{1}\left(L \Gamma_{2}(A) \otimes A\right) & \rightarrow L_{1} \Gamma_{3}(A) \\
\rightarrow & L_{3 n} \mathscr{L}^{3}(A, n) \rightarrow \Gamma_{2}(A) \otimes A
\end{aligned}
$$

We may now summarize this discussion as follows: 
Theorem 8.2 Case I $n$ is odd.

$L_{i} \mathscr{L}^{3}(A, n)$

$$
= \begin{cases}\operatorname{ker}\left\{\operatorname{Tor}\left(\Omega_{2}(A), A\right) \rightarrow \Omega_{3}(A)\right\} & i=3 n+2, \\ \operatorname{gr}_{1} L_{3 n+1} \mathscr{L}^{3}(A, n) & \\ =\operatorname{ker}\left\{\pi_{1}\left(L \Lambda^{2}(A) \otimes A\right) \rightarrow L_{1} \Lambda^{3}(A)\right\} & \\ \operatorname{gr}_{2} L_{3 n+1} \mathscr{L}^{3}(A, n) & \\ =\operatorname{coker}\left\{\operatorname{Tor}\left(\Omega_{2}(A), A\right) \rightarrow \Omega_{3}(A)\right\} & \\ Y^{3}(A) & i=3 n, \\ A \otimes \mathbb{Z} / 3 & n+3 \leq i<3 n-1, i \equiv n+3 \bmod 4, \\ \operatorname{Tor}(A, \mathbb{Z} / 3) & n+4 \leq i \leq 3 n-1, i \equiv n \bmod 4,\end{cases}
$$

and $L_{i} \mathscr{L}^{3}(A, n)=0$ for all other values of $i$.

Case II $n$ is even.

$L_{i} \mathscr{L}^{3}(A, n)$

$$
= \begin{cases}\operatorname{ker}\left\{\operatorname{Tor}\left(R_{2}(A), A\right) \rightarrow R_{3}(A)\right\} & i=3 n+2, \\ \operatorname{gr}_{1} L_{3 n+1} \mathscr{L}^{3}(A, n) & \\ =\operatorname{ker}\left\{\pi_{1}\left(L \Gamma_{2}(A) \otimes A\right) \rightarrow L_{1} \Gamma_{3}(A)\right\} & \\ \operatorname{gr}_{2} L_{3 n+1} \mathscr{L}^{3}(A, n) & \\ =\operatorname{coker}\left\{\operatorname{Tor}\left(R_{2}(A), A\right) \rightarrow R_{3}(A)\right\} & \\ \operatorname{Tor}(A, \mathbb{Z} / 3) \oplus \mathscr{L}^{3}(A) & i=3 n, \\ A \otimes \mathbb{Z} / 3 & n+3 \leq i \leq 3 n-1, i \equiv n+3 \bmod 4, \\ \operatorname{Tor}(A, \mathbb{Z} / 3) & n+4 \leq i<3 n-1, i \equiv n \bmod 4,\end{cases}
$$

and $L_{i} \mathscr{L}^{3}(A, n)=0$ for all other values of $i$.

Note that in the computation of $L_{3 n} \mathscr{L}^{3}(A, n)$ for $n$ odd, we relied on the surjectivity of the map (8-7).

Example The previous discussion shows in particular that

$$
L_{i} \mathscr{L}^{3}(\mathbb{Z} / 3,5)= \begin{cases}\mathbb{Z} / 9 & i=16, \\ \mathbb{Z} / 3 & i=8,9,12,13,17, \\ 0 & \text { otherwise. }\end{cases}
$$

A functorial description of some of these groups will be given in Lemma 11.2. 
For a given a free abelian group $A$ and an integer $n \geq 2$ the composite map

$$
\mathscr{L}^{n}(A) \rightarrow \otimes^{n}(A) \rightarrow \mathscr{L}^{n}(A)
$$

is simply multiplication by $n$ [45, Proposition 3.3]. It follows that for an odd prime $p$, the derived functors $L_{i} \mathscr{L}^{p}(\mathbb{Z}, n)$ are $p$-groups (see (8-1)). The torsion part of the derived functors can be usually be determined by general arguments. Recall that by Bousfield [7, Corollary 9.5], if $T: \mathrm{Ab} \rightarrow \mathrm{Ab}$ is a functor of finite degree which preserves direct limits, then $L_{q} T(A, n)$ is a torsion group for every abelian group $A$, unless $q$ is divisible by $n$.

The $p$-components of the derived functors of $\mathscr{L}^{p}$ and $J^{p}$ are connected by the following relation [45, Proposition 4.7]: for every prime $p$, there are natural isomorphisms

$$
\pi_{i}\left(\mathbb{Z} / p \stackrel{L}{\otimes} L \mathscr{L}^{p}(\mathbb{Z}, n)\right) \simeq \pi_{i}\left(\mathbb{Z} / p \stackrel{L}{\otimes} L J^{p}(\mathbb{Z}, n)\right), \quad i \geq 0, n \geq 2 .
$$

However the formulas for the full derived functors $L_{i} J^{p}(\mathbb{Z}, n)$ are more complicated than those for the functors $L_{i} \mathscr{L}^{p}(\mathbb{Z}, n)(8-1)$. For example, we know by Theorem 8.2 that

$$
L \mathscr{L}^{5}(\mathbb{Z}, 3) \simeq K(\mathbb{Z} / 5,10)
$$

so that by (4-3)

$$
L J^{2}(\mathbb{Z}, 3) \stackrel{L}{\otimes} L J^{3}(\mathbb{Z}, 3) \simeq K(\mathbb{Z} / 3,12) .
$$

On the other hand $L J^{3}(\mathbb{Z}, 3) \simeq K(\mathbb{Z} / 3,6)$ by $(8-1)$, and the values of the derived functors $L_{i} J^{5}(\mathbb{Z}, 3)$ now follow from those of $\mathscr{L}^{5}(\mathbb{Z}, 3)$ and the Curtis decomposition of $\mathscr{L}^{5}$. One finds

$$
L_{i} J^{5}(\mathbb{Z}, 3) \simeq \begin{cases}\mathbb{Z} / 3 & i=13, \\ \mathbb{Z} / 5 & i=10, \\ 0 & i \neq 10,13 .\end{cases}
$$

One can compute the groups $L_{i} J^{5}(\mathbb{Z}, n)$ for a general $n$ by similar methods. One finds that $L_{i} J^{5}(\mathbb{Z}, n)$ is isomorphic to $L_{i} \mathscr{L}^{5}(\mathbb{Z}, n)$ for $i \geq 1$ and even $n$, and that $L_{i} J^{5}(\mathbb{Z}, n)$ contains only 3 -torsion and 5 -torsion elements whenever $n$ is odd. A similar computation detects a nontrivial 11-torsion element in $L_{29} J^{13}(\mathbb{Z}, 3)$, whereas the corresponding groups $L_{i} \mathscr{L}^{13}(\mathbb{Z}, 3)$ are 13-torsion for all $i$.

\section{Derived functors of composite functors}

Consider a pair of composable functors

$$
\mathcal{A} \stackrel{G}{\longrightarrow} \mathcal{B} \stackrel{F}{\longrightarrow} \mathcal{C}
$$


between abelian categories, and in which the categories $\mathcal{A}$ and $\mathcal{B}$ have enough projectives. In addition, we assume that $G(A)$ is of finite projective dimension for each object $A \in \mathcal{A}$. When these functors are additive, the composite functor spectral sequence discussed for example in Weibel [47, Section 5.8] describes the derived functors of the composite functor $G \circ F$ in terms of those of $F$ and $G$, under the condition that the objects $G(P)$ are $F$-acyclic for any projective object $P$ of $\mathcal{A}$ (we will refer to this as the $F$-acyclicity hypothesis). We will now carry out a similar discussion when $G$ and $F$ are no longer additive, in which case chain complexes must be replaced by simplicial abelian groups.

Let $P_{*}$ be projective resolution of an object $A \in \mathcal{A}$. Following the notation of [47, Section 5.7], we construct a Cartan-Eilenberg resolution $\mathbb{P}_{* . *}$ of the simplicial object $G\left(P_{*}\right)$, with $\mathbb{P}_{p, *}^{B}$ (resp. $\mathbb{P}_{p, *}^{Z}$, resp. $\mathbb{P}_{p, *}^{H}$ ) the chosen projective resolution of $B_{p} G\left(P_{*}\right)$ (resp. $Z_{p} G\left(P_{*}\right)$, resp. $\left.L_{p} G(A)\right)$. By the Dold-Kan correspondence this yields in particular a projective bisimplicial resolution of $L G(A)$ ) which we will also denote by $\mathbb{P}_{*, *}$, as well as a corresponding projective simplicial model $\mathbb{P}_{p, *}^{H}$ for the Eilenberg-Mac Lane spaces $K\left(L_{p} G(A), p\right)$. The two filtrations on the complex associated to the bisimplicial object $F \mathbb{P}_{* . *}$ determine a pair of spectral sequences with common abutment $\pi_{n}(L F \circ L G(A))$. The initial terms of the first of these are given by

$$
E_{p, q}^{2}=L_{p+q} F\left(\bigoplus_{\sum_{i} q_{i}=q} K\left(L_{q_{i}} G(A), q_{i}\right)\right) .
$$

as in [6]. When the functor $F$ is of finite degree, we may decompose this initial term according to cross-effects of $F$, as in Dold and Puppe [20, Section 4.18], so that the spectral sequence can be expressed as

$$
\begin{aligned}
E_{p, q}^{2}=\bigoplus_{r \geq 1} \bigoplus_{q_{1}+\cdots+q_{r}=q} L_{p+q} F_{[r]}\left(L_{q_{1}} G(A), q_{1} \mid\right. & \left.\cdots \mid L_{q_{r}} G(A), q_{r}\right) \\
& \Longrightarrow \pi_{p+q}((L F \circ L G)(A)) .
\end{aligned}
$$

The $F$-acyclicity hypothesis, which here asserts that $L_{i} F(G(P))=0$ for any projective abelian group $P$ and all $i>0$, implies that the morphism $(L F \circ G)(P) \longrightarrow F G(P)$ is a quasi-isomorphism for any projective object $P$ in $A$, and so is the induced map

$$
(L F \circ L G)(A) \longrightarrow L(F G)(A) .
$$

The spectral sequence (9-2) can now be written as

$$
\begin{aligned}
& E_{p, q}^{2}=\bigoplus_{r \geq 1} \bigoplus_{q_{1}+\cdots+q_{r}=q} L_{p+q} F_{[r]}\left(L_{q_{1}} G(A), q_{1}|\cdots| L_{q_{r}} G(A), q_{r}\right) \\
& \Longrightarrow L_{p+q}(F G)(A) .
\end{aligned}
$$


Replacing the object $A$ by the shifted derived category object $A[n]$, in other words by the Eilenberg-Mac Lane object $K(A, n)$, we may now compute under the same hypotheses the derived functors $L_{r}(F G)(A, n)$ for all $n$. The $F$-acyclicity hypothesis implies inductively that the quasi-isomorphism (9-3) determines a quasi-isomorphism

$$
(L F \circ L G)(A, n) \longrightarrow L(F G)(A, n)
$$

for all $n \geq 0$, since we can choose as a simplicial model for $K(A, n)$ the bisimplicial model

$$
\cdots \longrightarrow K\left(A^{3}, n-1\right) \longrightarrow K\left(A^{2}, n-1\right) \longrightarrow K(A, n-1) \longrightarrow\{e\}
$$

and work componentwise. Since no change is necessary in the discussion of spectral sequence (9-2) when passing from the $n=0$ case to the general situation, we finally obtain for any positive $n$, when $F$ is of finite degree and the $F$-acyclicity hypothesis is satisfied, a functorial spectral sequence:

$$
\begin{array}{r}
E_{p, q}^{2}=\bigoplus_{r \geq 1} \bigoplus_{q_{1}+\cdots+q_{r}=q} L_{p+q} F_{[r]}\left(L_{q_{1}} G(A, n), q_{1}|\cdots| L_{q_{r}} G(A, n), q_{r}\right) \\
\Longrightarrow L_{p+q}(F G)(A, n) .
\end{array}
$$

We now restrict ourselves to a special case, that in which $F$ and $G$ are endo-functors on the category of abelian groups (or more generally the category of $R$-modules, with $R$ a principal ideal domain or even a hereditary ring). By construction, the total complex of $\mathbb{P}_{*, *}$ and the complex $\bigoplus_{q} \mathbb{P}_{q, *}^{H}$ are both projective and have as homology $\bigoplus_{q} L_{q} G(A)[q]$, viewed as a complex with trivial differentials. It follows as in Dold [19, Section II.4] that this identification of their homology may be realized by a chain homotopy equivalence between the complexes, which in turn induces a simplicial homotopy equivalence between the corresponding simplicial groups $\mathbb{P}$ and $\bigoplus_{q} \mathbb{P}_{q, *}^{H}$. The induced homotopy equivalence between $F(\mathbb{P})$ and $F\left(\mathbb{P}_{q, *}^{H}\right)$ makes it clear that in this case the $E^{2}$ term of the spectral sequence (9-6) is (noncanonically) isomorphic to its abutment. It follows that the spectral sequence degenerates at the $E^{2}$ level, so that this proves the following proposition:

Proposition 9.1 Let $F$ and $G$ be a pair of endofunctors on the category of abelian groups, with $F$ of finite degree. Suppose that for any projective abelian group $P$, $L_{q} F(G(P))=0$ whenever $q>0$. Then the spectral sequence (9-6) degenerates at $E^{2}$, and the graded components associated to the filtration on the abutment $L_{m}(F G)(A)$ of the spectral sequence are described by the formula

$$
\begin{aligned}
\operatorname{gr}_{p} L_{p+q} & (F G)(A, n) \\
& \simeq \bigoplus_{r \geq 1} \bigoplus_{q_{1}+\cdots+q_{r}=q} L_{p+q} F_{[r]}\left(L_{q_{1}} G(A, n), q_{1}|\cdots| L_{q_{r}} G(A, n), q_{r}\right) .
\end{aligned}
$$


When $F$ is one of the functor $\mathrm{SP}^{s}, \Lambda^{s}$ or $\Gamma_{s}$, such an assertion may also be deduced, under the $F$-acyclicity hypothesis, from formula $\mathrm{V}$ (4.2.7) of Illusie [29].

\subsection{The derived functors of $\Lambda^{2} \Lambda^{2}$}

As an illustration of Proposition 9.1, we will now compute the derived functors of the functor $L_{i}\left(\Lambda^{2} \Lambda^{2}\right)(A, n)$ for all for $n=0,1,2$. Such results are of interest to us, since $\Lambda^{2} \Lambda^{2}$ is the first composite functor arising in the decomposition (7-9) of the Lie functors $\mathscr{L}^{n} A$.

We know by (4-3) that

$$
L_{i} \Lambda^{2}(A)=\left\{\begin{array}{ll}
\Omega_{2}(A) & i=1, \\
\Lambda^{2}(A) & i=0, \\
0 & \text { else, }
\end{array} \quad L_{i} \Lambda^{2}(A, 1)= \begin{cases}R_{2}(A) & i=3 \\
\Gamma_{2}(A) & i=2 \\
0 & \text { else }\end{cases}\right.
$$

$\begin{aligned} \text { (9-8) } L_{i} \Lambda^{2}(A, 2) & =\begin{array}{ll}\Omega_{2}(A) & i=5, \\ \lambda^{2}(A) & i=4, \\ A \otimes \mathbb{Z} / 2 & i=3, \\ 0 & \text { else, }\end{array} \quad L_{i} \Lambda^{2}(A, 3)= \begin{cases}R_{2}(A) & i=7, \\ \Gamma_{2}(A) & i=6, \\ \operatorname{Tor}(A, \mathbb{Z} / 2) & i=5, \\ A \otimes \mathbb{Z} / 2 & i=4, \\ 0 & \text { else, }\end{cases} \\ L_{i} \Lambda^{2}(A, 4) & =\left\{\begin{array}{ll}\Omega_{2}(A) & i=9, \\ \lambda^{2}(A) & i=8, \\ \operatorname{Tor}(A, \mathbb{Z} / 2) & i=6, \\ A \otimes \mathbb{Z} / 2 & i=5,7, \\ 0 & \text { else, }\end{array} \quad L_{i} \Lambda^{2}(A, 5)= \begin{cases}R_{2}(A) & i=11, \\ \Gamma_{2}(A) & i=10, \\ \operatorname{Tor}(A, \mathbb{Z} / 2) & i=7,9, \\ A \otimes \mathbb{Z} / 2 & i=6,8, \\ 0 & \text { else, }\end{cases} \right.\end{aligned}$

with $\lambda^{2}(A)$ defined in (4-1). Proposition 9.1 yields Table 2 below for the functors $L_{i} \Lambda^{2} \Lambda^{2}(A)$.

The functors $L_{n}\left(\Lambda^{2} \Lambda^{2}\right)(A)$ can be read off from the $p+q=n$ line of Table 2. In particular, there is a (nonnaturally split) short exact sequence

$$
0 \longrightarrow \Lambda^{2}(A) \otimes \Omega_{2}(A) \longrightarrow L_{1}\left(\Lambda^{2} \Lambda^{2}\right)(A) \longrightarrow \Omega_{2} \Lambda^{2}(A) \longrightarrow 0
$$

which may be viewed as a symmetrized version of a Künneth formula for the expression

$$
\pi_{1}\left(L \Lambda^{2}(A) \stackrel{L}{\otimes} L \Lambda^{2}(A)\right) .
$$




\begin{tabular}{r|cc}
2 & 0 & $R_{2} \Omega_{2}(A)$ \\
1 & $\Omega_{2} \Lambda^{2}(A)$ & $\Gamma_{2} \Omega_{2}(A) \oplus \operatorname{Tor}\left(\Lambda^{2}(A), \Omega_{2}(A)\right)$ \\
$p=0$ & $\Lambda^{2} \Lambda^{2}(A)$ & $\Lambda^{2} A \otimes \Omega^{2}(A)$ \\
\hline & $q=0$ & 1
\end{tabular}

Table 2: $\operatorname{gr}_{p}\left(L_{p+q}\left(\Lambda^{2} \Lambda^{2}\right)(A)\right)$

We now pass to the derived functors $L_{n}\left(\Lambda^{2} \Lambda^{2}(A, 1)\right)$. The values of these derived functors are displayed in Table 3. They are obtained as above from the values (9-8) of the derived functors of $\Lambda^{2}$.

\begin{tabular}{r|cccc}
4 & 0 & $R_{2} R_{2}(A)$ & 0 & 0 \\
3 & $\Omega_{2} \Gamma_{2}(A)$ & $\Gamma_{2} R_{2}(A)$ & 0 & 0 \\
2 & $\lambda^{2} \Gamma_{2}(A)$ & $\operatorname{Tor}\left(R_{2}(A), \mathbb{Z} / 2\right)$ & 0 & 0 \\
1 & $\Gamma_{2}(A) \otimes \mathbb{Z} / 2$ & $R_{2}(A) \otimes \mathbb{Z} / 2$ & 0 & $\operatorname{Tor}\left(\Gamma_{2}(A), R_{2}(A)\right)$ \\
$p=0$ & 0 & 0 & 0 & $\Gamma_{2}(A) \otimes R_{2}(A)$ \\
\hline & $q=2$ & 3 & 4 & 5
\end{tabular}

Table 3: $\operatorname{gr}_{p}\left(L_{p+q}\left(\Lambda^{2} \Lambda^{2}\right)(A, 1)\right)$

When one also takes into account the values of $L_{i} \Lambda(A, n)$ for $n=4,5$, one finds the values for the derived functors of $\Lambda^{2} \Lambda^{2}(A, 2)$ displayed in Table 4.

\subsection{The derived functors of $\Lambda^{2} \Gamma_{2}$}

We will now carry out a similar discussion for the derived functors of $\Lambda^{2} \Gamma_{2}$. By (4-4),

$$
L_{i} \Gamma_{2}(A)=\left\{\begin{array}{ll}
R_{2}(A) & i=1, \\
\Gamma_{2}(A) & i=0, \\
0 & i \neq 0,1,
\end{array} \quad L_{i} \Gamma_{2}(A, 1)= \begin{cases}\Omega_{2}(A) & i=3, \\
\lambda^{2}(A) & i=2, \\
A \otimes \mathbb{Z} / 2 & i=1, \\
0 & i \neq 1,2,3 .\end{cases}\right.
$$




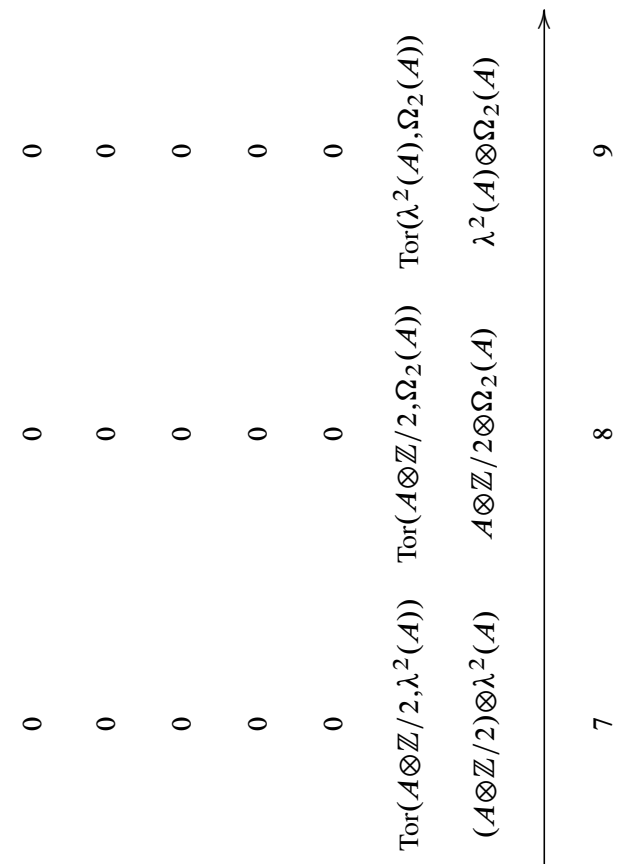

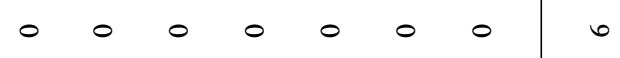

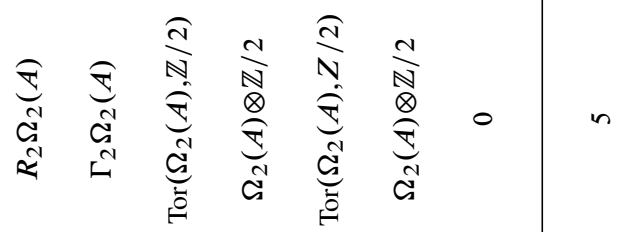

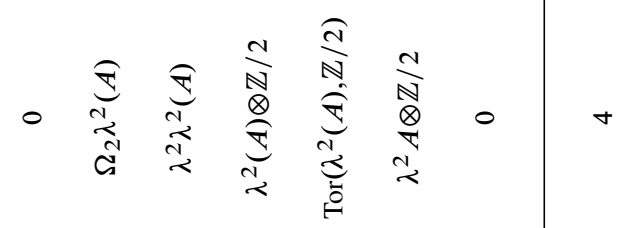

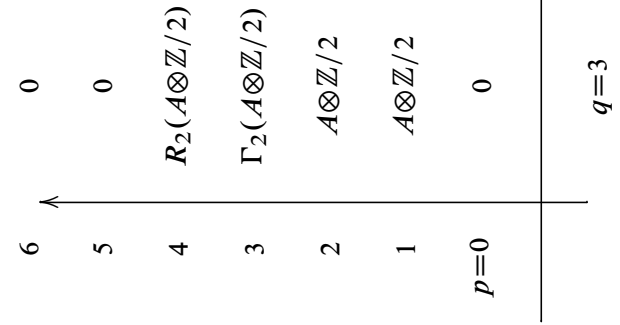

Table 4: $\operatorname{gr}_{p}\left(L_{p+q}\left(\Lambda^{2} \Lambda^{2}\right)(A, 2)\right)$ 
Since $\Gamma_{2}(P)$ is torsion-free for any torsion-free group $P$, the derived functors of $\Lambda^{2} \Gamma_{2}$ may be computed by formula (9-7). Tables 5 and 6 below may now be respectively deduced from formulas (9-8) and (9-9).

\begin{tabular}{c|cc}
2 & $R_{2} R_{2}(A)$ \\
1 & $\Omega_{2} \Gamma_{2}(A)$ & $\Gamma_{2} R_{2}(A) \oplus \operatorname{Tor}\left(\Gamma_{2}(A), R_{2}(A)\right)$ \\
$p=0$ & $\Lambda^{2} \Gamma_{2}(A)$ & $\Gamma_{2}(A) \otimes R_{2}(A)$ \\
\hline & $q=0$ & 1
\end{tabular}

Table 5: $\operatorname{gr}_{p}\left(L_{p+q}\left(\Lambda^{2} \Gamma_{2}\right)(A)\right)$

\begin{tabular}{c|ccccc}
4 & 0 & 0 & $R_{2} \Omega_{2}(A)$ & 0 & 0 \\
3 & 0 & $\Omega_{2} \lambda^{2}(A)$ & $\Gamma_{2} \Omega_{2}(A)$ & 0 & 0 \\
2 & $R_{2}(A \otimes \mathbb{Z} / 2)$ & $\lambda^{2} \lambda^{2}(A)$ & $\operatorname{Tor}\left(\Omega_{2}(A), \mathbb{Z} / 2\right)$ & 0 & 0 \\
1 & $\Gamma_{2}(A \otimes \mathbb{Z} / 2)$ & $\lambda^{2}(A) \otimes \mathbb{Z} / 2$ & $\Omega_{2}(A) \otimes \mathbb{Z} / 2$ & $\operatorname{Tor}\left(A \otimes \mathbb{Z} / 2, \lambda^{2}(A)\right)$ & $\operatorname{Tor}\left(A \otimes \mathbb{Z} / 2, \Omega_{2}(A)\right)$ \\
0 & 0 & 0 & $A \otimes \mathbb{Z} / 2 \otimes \lambda^{2}(A)$ & $A \otimes \mathbb{Z} / 2 \otimes \Omega_{2}(A)$ & $\lambda^{2}(A) \otimes \Omega_{2}(A)$ \\
\hline & $q=1$ & 2 & 3 & 4 & 5
\end{tabular}

Table 6: $\operatorname{gr}_{p}\left(L_{p+q}\left(\Lambda^{2} \Gamma_{2}\right)(A, 1)\right)$

The coincidence, up to changes in degree, between certain terms in Table 5 and those in Table 3, and (more strikingly) between certain terms in Table 6 and those in Table 4 is explained by the décalage isomorphisms (2-40) between the derived functors of $\Gamma_{2}$ and those of $\Lambda^{2}$.

\section{Derived functors of super-Lie functors}

In view of (7-19), the décalage isomorphisms (2-42) and formulas (8-2) imply (for $n \geq 1$ ):

$$
L_{n+k} \mathscr{L}_{s}^{3}(\mathbb{Z}, n)= \begin{cases}\mathbb{Z} / 3 & k=4 i+1, i=0,1, \ldots,\left[\frac{n-1}{2}\right], \\ 0 & \text { otherwise. }\end{cases}
$$

We will now examine the relations between the derived functors of $\mathscr{L}^{n}$ and $\mathscr{L}_{s}^{n}$. For any free simplicial abelian group $A_{*}$, the maps $\chi_{n}$ and $\bar{\chi}_{n}$ (7-32) induce arrows:

$$
\begin{array}{ll}
\chi_{n}^{*}: \pi_{m}\left(L \mathscr{L}_{s}^{n}\left(A_{*}\right) \otimes L \Lambda^{n}(\mathbb{Z}, 1)\right) \rightarrow \pi_{m}\left(L \mathscr{L}^{n}(A) \otimes \mathbb{Z}[1]\right), & m \geq 0 \\
\bar{\chi}_{n}^{*}: \pi_{m}\left(L \mathscr{L}^{n}\left(A_{*}\right) \otimes L \Lambda^{n}(\mathbb{Z}, 1)\right) \rightarrow \pi_{m}\left(L \mathscr{L}_{s}^{n}(A) \otimes \mathbb{Z}[1]\right), & m \geq 0
\end{array}
$$


For $A_{*}=K(A, m)$, these determine by adjunction pension maps

$$
\begin{aligned}
& \chi_{n}^{*}: L_{m} \mathscr{L}_{s}^{n}(A, k) \rightarrow L_{m+n} \mathscr{L}^{n}(A, k+1) \\
& \bar{\chi}_{n}^{*}: L_{m} \mathscr{L}^{n}(A, k) \rightarrow L_{m+n} \mathscr{L}_{s}^{n}(A, k+1) .
\end{aligned}
$$

which may be viewed as generalized décalage transformations, even though the maps $\chi_{n}^{*}$ are no longer isomorphisms. We will for this reason refer to such maps as semidécalage morphisms. Similarly, the pairing $\beta_{n}$ (7-36) determines a family of pension isomorphisms (2-42) which we now denote $\zeta_{n}$ :

$$
L_{m} Y^{n}(A, k) \stackrel{\zeta_{n}}{\longrightarrow} L_{m+n} J^{n}(A, k+1) .
$$

Proposition 7.7 now implies the following assertion:

Theorem 10.1 The following diagram is commutative:

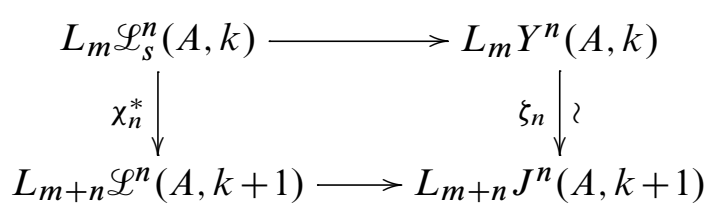

Let us now consider the boundary maps:

$$
\begin{aligned}
& \theta_{m}: L_{m} J^{n}(A, k) \rightarrow L_{m-1} \widetilde{J}^{n}(A, k), \\
& \bar{\theta}_{m}: L_{m} Y^{n}(A, k) \rightarrow L_{m-1} \tilde{Y}^{n}(A, k)
\end{aligned}
$$

induced by the short exact sequences (2-9) and (7-28). The following proposition is a corollary of Theorem 10.1:

Proposition 10.2 For $m, n \geq 1$ and any abelian group $A$, the following diagram commutes (where the lower left-hand arrow is $\theta^{m+n+1}$ and the vertical arrows are décalage and semi-décalage morphisms):

$(10-6)$

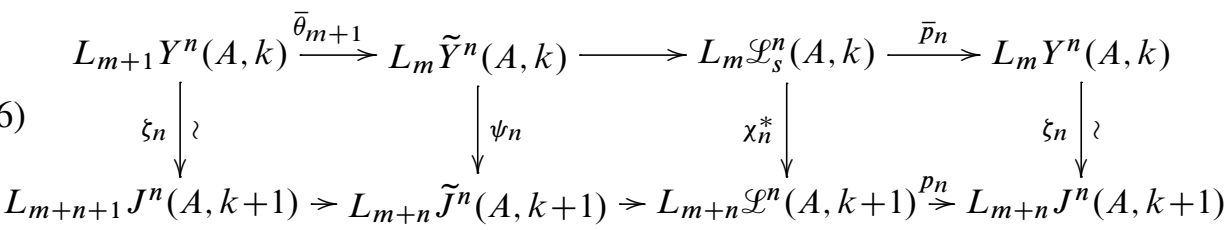


We now suppose that $n \geq 2$. The following diagram, in which the vertical arrows are décalage maps, is commutative:

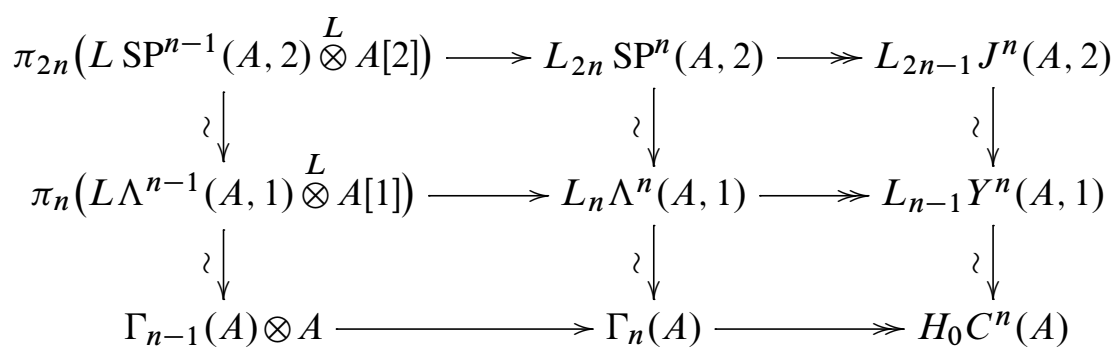

By Proposition 3.1, it now follows in particular that there exists a natural isomorphism

$$
L_{2 n-1} J^{n}(A, 2) \simeq \bigoplus_{p \mid n} \Gamma_{n / p}(A \otimes \mathbb{Z} / p),
$$

which describes explicitly the right-hand terms in diagram (10-7).

\subsection{The fourth Lie and super-Lie functors}

We will now discuss certain derived functors of the functors $\mathscr{L}^{4}$ and $\mathscr{L}_{s}^{4}$. Recall that by (7-10) and (7-29),

$$
\tilde{J}^{4}(A) \simeq \Lambda^{2} \Lambda^{2}(A), \quad \tilde{Y}^{4}(A) \simeq \Lambda^{2} \Gamma_{2}(A) .
$$

for any free abelian $A$. By (10-8), the right-hand vertical arrows in diagram (10-7) for $n=4$ are

$$
L_{7} J^{4}(A, 2) \stackrel{\sim}{\longrightarrow} L_{3} Y^{4}(A, 1) \stackrel{\sim}{\longrightarrow} \Gamma_{2}(A \otimes \mathbb{Z} / 2) .
$$

Proposition 10.3 For every abelian group $A$, the arrow

$$
L_{3} Y^{4}(A, 1) \stackrel{\bar{\theta}_{3}}{\longrightarrow} L_{2} \Lambda^{2} \Gamma_{2}(A, 1)
$$

is a natural isomorphism between a pair of functors, both naturally isomorphic to $\Gamma_{2}(A \otimes \mathbb{Z} / 2)$.

Proof Let us first verify that the map $\bar{\theta}_{3}$ (10-10) is surjective. Consider the simplicial model (2-17) of $L \Lambda^{2} \Gamma_{2}(A, 1)$ determined by a flat resolution (2-15) of $A$. We define a map

$$
\Gamma_{2}(A \otimes \mathbb{Z} / 2) \stackrel{v}{\longrightarrow} L_{2} \Lambda^{2} \Gamma_{2}(A, 1)
$$


explicitly as follows:

$$
\begin{array}{ccc}
\Gamma_{2}(A \otimes \mathbb{Z} / 2) & \rightarrow \Lambda^{2} \Gamma_{2}\left(A_{1} \oplus s_{0}\left(A_{0}\right) \oplus s_{1}\left(A_{0}\right)\right) / \partial_{0}\left(\operatorname{ker}\left(\partial_{1}\right) \cap \operatorname{ker}\left(\partial_{2}\right) \cap \operatorname{ker}\left(\partial_{3}\right)\right) \\
\gamma_{2}(\bar{a}) & \mapsto & \gamma_{2}\left(s_{0}(a)\right) \wedge \gamma_{2}\left(s_{1}(a)\right)
\end{array}
$$

for some lift $a$ to $M$ of $\bar{a} \in A \otimes \mathbb{Z} / 2$. Under the natural transformation $\Lambda^{2} \Gamma_{2} \longrightarrow \mathscr{L}_{s}^{4}$ (7-26), the image of $\gamma_{2}(\bar{a})$ goes to the element

$$
\left\{s_{o}(a), s_{1}(a), s_{0}(a), s_{1}(a)\right\}
$$

in the term $\mathscr{L}_{s}^{4}\left(L \oplus s_{0}(M) \oplus s_{1}(M)\right)$ of the corresponding simplicial model for $L \mathscr{L}_{s}^{4}(A, 1)$. The element

$$
\begin{aligned}
\tau:=\left\{s_{1} s_{0}(a), s_{2} s_{0}(a), s_{1} s_{0}(a), s_{2} s_{0}(a)\right\}-\left\{s_{1} s_{0}(a), s_{2} s_{0}(a), s_{1} s_{0}(a), s_{2} s_{1}(a)\right\} \\
\in \mathscr{L}_{s}^{4}\left(s_{0}\left(A_{1}\right) \oplus s_{1}\left(A_{1}\right) \oplus s_{2}\left(A_{1}\right) \oplus s_{1} s_{0}\left(A_{0}\right) \oplus s_{2} s_{0}\left(A_{0}\right) \oplus s_{2} s_{1}\left(A_{0}\right)\right)
\end{aligned}
$$

satisfies the equations $\partial_{i}(\tau)=0, i=1,2,3$ and

$$
\partial_{0}(\tau)=\left\{s_{0}(a), s_{1}(a), s_{0}(a), s_{1}(a)\right\} \in \mathscr{L}_{s}^{4}\left(A_{1} \oplus s_{0}\left(A_{0}\right) \oplus s_{1}\left(A_{0}\right)\right) .
$$

It follows that the map $L_{2} \Lambda^{2} \Gamma_{2}(A, 1) \rightarrow L_{2} \mathscr{L}_{\underline{s}}^{4}(A, 1)$ is trivial so that, by exactness of the upper line of diagram (10-6), the arrow $\bar{\theta}_{3}$ is surjective.

We will now give a more explicit description of the target of $\bar{\theta}_{3}$. We have a natural isomorphism

$$
v: L_{2} \Lambda^{2} \Gamma_{2}(A, 1) \stackrel{\sim}{\longrightarrow} \Gamma_{2}(A \otimes \mathbb{Z} / 2)
$$

since the only nontrivial term of total degree 2 in Table 6 is the expression $\Gamma_{2}(A \otimes \mathbb{Z} / 2)$ in bidegree $(1,1)$. We now have a pair of arrows $u$ (10-9) and $v$, which provide natural isomorphisms between both the source and target of $\bar{\theta}_{3}$ and the group $\Gamma_{2}(A \otimes \mathbb{Z} / 2)$. We may now assume that $A$ is finitely generated. In that case both source and target of the surjective map $\bar{\theta}_{3}$ are finite groups of the same order, so $\bar{\theta}_{3}$ is an isomorphism.

We know by the description of homotopy groups of $L \Lambda^{2} \Lambda^{2}(A, 2)$ in Table 4 that there is a natural projection $L_{6} \Lambda^{2} \Lambda^{2}(A, 2) \rightarrow \Gamma_{2}(A \otimes \mathbb{Z} / 2)$. The following proposition is a consequence of Proposition 10.2 and Proposition 10.3:

Proposition 10.4 The group $G:=L_{6} \Lambda^{2} \Lambda^{2}(A, 2)$ is endowed with a 3-step descending filtration $F^{i} G(1 \leq i \leq 3)$ for which the associated graded components are described by

$$
\operatorname{gr}_{i} G= \begin{cases}\Gamma_{2}(A \otimes \mathbb{Z} / 2) & i=1 \\ \operatorname{Tor}\left(\lambda^{2}(A), \mathbb{Z} / 2\right) & i=2 \\ \Omega_{2}(A) \otimes \mathbb{Z} / 2 & i=3\end{cases}
$$


In addition, the projection $w$ of $G$ onto the highest graded component $\operatorname{gr}_{1} G$ is the map arising from the edge-homomorphism in the spectral sequence (9-6) described in Table 4. This surjection $w$ is split, up to an isomorphism, by the boundary map $L_{7} J^{4}(A, 2) \stackrel{\theta_{7}}{\longrightarrow} L_{6} \Lambda^{2} \Lambda^{2}(A, 2)$ provided by the decomposition (7-9) of $\mathscr{L}^{4}(A)$.

Proof The associated graded terms $\Omega_{2}(A) \otimes \mathbb{Z} / 2$ and $\operatorname{Tor}\left(\lambda^{2}(A), \mathbb{Z} / 2\right)$ in the line $p+q=6$ of Table 4 give us the required description of $F^{2} G=\operatorname{ker}(w)$. The previous discussion provides us with a commutative diagram

$$
\begin{aligned}
& \Gamma_{2}(A \otimes \mathbb{Z} / 2) \underset{\imath}{\stackrel{\simeq}{\simeq}} L_{3} Y^{4}(A, 1) \underset{\bar{\theta}_{3}}{\stackrel{\simeq}{\simeq}} L_{2}\left(\Lambda^{2} \Gamma_{2}\right)(A, 1) \\
& L_{7} J^{4}(A, 2) \stackrel{\theta_{7}}{\longrightarrow} L_{6}\left(\Lambda^{2} \Lambda^{2}\right)(A, 2) \stackrel{w}{\longrightarrow} \Gamma_{2}(A \otimes \mathbb{Z} / 2)
\end{aligned}
$$

where the injectivity of the map $\psi_{4}(10-6)$ is obtained by examining the behavior of the decompositions (9-7) of its source and target under décalage. It remains to show that the composite map

$$
w \circ \theta_{7}: L_{7} J^{4}(A, 2) \rightarrow \Gamma_{2}(A \otimes \mathbb{Z} / 2)
$$

is an isomorphism. When $A$ is free abelian of finite rank, we have $L_{6} \Lambda^{2} \Lambda^{2}(A, 2) \simeq$ $\Gamma_{2}(A \otimes \mathbb{Z} / 2)$, so that the injective map

$$
\psi_{4}: \Gamma_{2}(A \otimes \mathbb{Z} / 2) \hookrightarrow L_{6} \Lambda^{2} \Lambda^{2}(A, 2)
$$

is a monomorphism between two finite groups of the same order. It follows that the map $w \circ \theta_{7}$ is an isomorphism whenever $A$ is free abelian, and therefore an epimorphism for an arbitrary abelian group $A$. Returning to the case of an abelian group $A$ of finite rank, we conclude that the epimorphism $w \circ \theta_{7}$ is an isomorphism, since source and target are finite groups of the same order. This implies that the corresponding assertion is true for an arbitrary abelian group.

In the sequel, we will also need the following result, which follows since the only nontrivial terms contributed by the Curtis decomposition to $L_{i} \mathscr{L}^{4}(A, 2)$ for $i<7$ are those provided by the derived functors of $\Lambda^{2} \Lambda^{2}$ :

Corollary 10.5 The group $L_{6} \mathscr{L}^{4}(A, 2)$ is canonically isomorphic to the direct sum of the two following expressions:

$$
\begin{aligned}
& \operatorname{gr}_{1} L_{6} \mathscr{L}^{4}(A, 2)=\operatorname{Tor}\left(\lambda^{2}(A), \mathbb{Z} / 2\right)=\operatorname{Tor}\left(\Lambda^{2}(A), \mathbb{Z} / 2\right) \oplus \operatorname{Tor}_{2}(A, \mathbb{Z} / 2, \mathbb{Z} / 2) \\
& \operatorname{gr}_{2} L_{6} \mathscr{L}^{4}(A, 2)=\Omega_{2}(A) \otimes \mathbb{Z} / 2 .
\end{aligned}
$$




\section{Homotopical applications}

\subsection{Moore spaces and the Curtis spectral sequence}

In this section we will review the Curtis spectral sequence, which will be our main tool for homotopical applications of our theory. Recall that for any abelian group $A$ and $n \geq 2$, a Moore space in degree $n$ is defined to be a simply connected space $X$ with $H_{i}(X) \simeq A$ for $i=n$, and $\tilde{H}_{i}(X)=0, i \neq n$. Such a Moore space will be denoted $M(A, n)$ when in addition an isomorphism $H_{n}(X) \simeq A$ is fixed. The homotopy type of $M(A, n)$ is determined by the pair $(A, n)$, since homology equivalence implies homotopy equivalence for simply connected spaces. When $A$ is free abelian with a chosen basis, a Moore space $M(A, n)$ can be constructed as a wedge of $n$-spheres, labelled by basis elements of $A$. For an arbitrary abelian group $A$ and $n \geq 2$, an $n$-dimensional Moore space is constructed as follows: choose a 2-step free resolution (2-15) of $A$ with chosen bases. $M(A, n)$ can then be defined as the mapping cone (see Gabriel and Zisman [27, VI 2]) of the induced map between the wedges of spheres $M(L, n) \rightarrow M(M, n)$. For any homomorphism of abelian groups $f: A \rightarrow B$, it is possible to construct a map $\phi: M(A, n) \rightarrow M(B, n)$ such that $H_{n}(\phi)=f$. However, the construction of the map $\phi$ is not canonical and the construction of Moore spaces is non-functorial. The canonical class in $H^{n}(M(A, n), A)$ induces a map

$$
M(A, n) \longrightarrow K(A, n)
$$

which is well-defined up to homotopy.

We will now recall the construction of the Curtis spectral sequence. Let $G$ be a simplicial group. The lower central series filtration on $G$ gives rise to the long exact sequence

$$
\pi_{i+1}\left(G / \gamma_{r}(G)\right) \rightarrow \pi_{i}\left(\gamma_{r}(G) / \gamma_{r+1}(G)\right) \rightarrow \pi_{i}\left(G / \gamma_{r+1}(G)\right) \rightarrow \pi_{i}\left(G / \gamma_{r}(G)\right) \rightarrow \cdots
$$

This exact sequence defines a graded exact couple, which gives rise to a natural spectral sequence $E(G)$ with the initial terms

$$
E_{r, q}^{1}(G)=\pi_{q}\left(\gamma_{r}(G) / \gamma_{r+1}(G)\right)
$$

and differentials

$$
d_{r, q}^{i}: E_{r, q}^{i}(G) \rightarrow E_{r+i, q-1}^{i}(G) .
$$

According to Curtis [15], for $K$ a connected simplicial set and $G=G K$ the associated Kan construction as in May [37, Section 26], this spectral sequence $E^{i}(G)$ converges to $E^{\infty}(G)$ and $\bigoplus_{r} E_{r, q}^{\infty}$ is the graded group associated to the filtration on $\pi_{q}(G K)$ 
induced by the lower central series filtration on $K$. Since $G K$ is a loop group of $K$, this spectral sequence may be written as

$$
E_{r, q}^{1}(K):=\pi_{q}\left(\gamma_{r}(G K) / \gamma_{r+1}(G K)\right) \Longrightarrow \pi_{q+1}(|K|) .
$$

The groups $E^{1}(G)$ are homology invariants of $K$. By the Magnus-Witt isomorphism (2-4), the spectral sequence (11-3) can be rewritten as

$$
E_{r, q}^{1}(K)=\pi_{q}\left(\mathscr{L}^{r}(\widetilde{\mathbb{Z}} K,-1)\right) \Longrightarrow \pi_{q+1}(|K|) .
$$

since the abelianization $G K_{\mathrm{ab}}:=G K / \gamma_{2} G K$ of $G K$ corresponds to the reduced chains $\widetilde{\mathbb{Z}} K$ on $K$, with degree shifted by 1 . When $K=M(A, n), \widetilde{\mathbb{Z}} K$ corresponds to an Eilenberg-Mac Lane space $K(A, n)$ so that the spectral sequence is simply of the form

$$
E_{r, q}^{1}=L_{q} \mathscr{L}^{r}(A, n-1) \Longrightarrow \pi_{q+1}(M(A, n))
$$

In particular,

$$
E_{1, q}^{1}=\pi_{q}(K(A, n-1))= \begin{cases}A & q=n-1 \\ 0 & q \neq n-1\end{cases}
$$

For more information regarding this spectral sequence, see Curtis [15] and Mikhailov and Passi [40, Chapter 5].

\subsection{The 3-torsion of $\pi_{n}\left(S^{2}\right)$}

As a first illustration of our techniques, we will now discuss the 3-torsion components of the homotopy groups of the sphere $S^{2}$. For this, consider the 3 -torsion parts of the various terms in the spectral sequence (11-5), with $A=\mathbb{Z}$ and $n=2$ :

$$
E_{r, q}^{1}=L_{q} \mathscr{L}^{r}(\mathbb{Z}, 1) \Rightarrow \pi_{q+1}\left(S^{2}\right) .
$$

From now on, we will denote by ${ }_{p} A$ the $p$-torsion subgroup of an abelian group $A$ and by ${ }_{(p)} A$ the quotient of $A$ by the $q$-torsion elements, for all primes $q \neq p$. We will refer to this quotient group as the $(p)$-torsion group of $A$.

It is shown in [15] (see also [40, Propositions 5.33 and 5.35]) that

$$
L_{i} J^{n}(\mathbb{Z}, 1)= \begin{cases}\mathbb{Z} & i=2, n=2, \\ 0 & \text { otherwise. }\end{cases}
$$

This, together with the Curtis decomposition (7-9) of the Lie functors and the computation of the groups $L_{i} \Lambda^{2} \Lambda^{2}(\mathbb{Z}, 1)$ in Table 3, implies that there is no 3 -torsion in any of the expressions $L_{q} \mathscr{L}^{p}(\mathbb{Z}, 1)$ for $p<6$. Let us show that the first nontrivial 3 -torsion term in the spectral sequence (11-6) occurs in the group $L_{5} \mathscr{L}^{6}(\mathbb{Z}, 1)$. It 
follows from (11-7) and the Künneth formula that no 3-torsion is produced by either of the factors $J^{6}(\mathbb{Z}, 1)$ and $J^{4}(\mathbb{Z}, 1) \otimes J^{2}(\mathbb{Z}, 1)$ of $\mathscr{L}^{6}(\mathbb{Z}, 1)$, nor is any contribution made by $J^{2} J^{3}(\mathbb{Z}, 1)$ since $J^{3}(\mathbb{Z}, 1)$ is contractible. It thus follows from (11-7) and $(8-2)$ (or $(8-14))$ that

$$
L_{i} \mathscr{L}^{6}(\mathbb{Z}, 1) \simeq L_{i} J^{3} J^{2}(\mathbb{Z}, 1) \simeq L_{i} \mathscr{L}^{3}(\mathbb{Z}, 2) \simeq \begin{cases}\mathbb{Z} / 3 & i=5, \\ 0 & i \neq 5 .\end{cases}
$$

We restate this result as

$$
L J^{3} J^{2}(\mathbb{Z}, 1) \simeq K(\mathbb{Z} / 3,5) .
$$

More generally, the Curtis decomposition (7-9), together with (11-7) and (10-8), implies that 3-torsion in the groups $L_{q} \mathscr{L}^{r}(\mathbb{Z}, 1)$ can only arise from components of the decomposition of the form $\mathrm{FJ}^{3^{k}} J^{2}$ and their tensor products (for functors $F=\mathrm{SP}^{k}$ or $F=J^{k}$ ), so that there is no 3 -torsion in the initial terms of (11-6) unless $6 \mid r$. The analysis of the $r=18$ case is similar to that of $r=6$. The only contribution to the 3 -torsion in $L_{q} \mathscr{L}^{r}(\mathbb{Z}, 1)$, for $q \leq 14$, comes from the derived functors of $J^{3} J^{3} J^{2}(\mathbb{Z}, 1)$, and by (11-9)

$$
L_{i} J^{3} J^{3} J^{2}(\mathbb{Z}, 1) \simeq L_{i} \mathscr{L}^{3}(\mathbb{Z} / 3,5) .
$$

These groups were computed in (8-16), so it now follows from the connectivity result (3-6) that

and

$$
\begin{array}{ll}
{ }_{3} L_{q} \mathscr{L}^{r}(\mathbb{Z}, 1)=\mathbb{Z} / 3, & r=18, q=8,9 \\
{ }_{3} L_{q} \mathscr{L}^{r}(\mathbb{Z}, 1)=0, & r \neq 18,5<q<10 .
\end{array}
$$

We refer to Mikhailov and Passi [40, Chapter 5] for a similar analysis of the 2-torsion components in the spectral sequence (11-6).

For $r \neq 12$, the 3 -torsion components of $L_{q} \mathscr{L}^{r}(\mathbb{Z}, 1)$ may all be computed by the previous method so long as $q \leq 14$, and indeed all of these components are trivial except for those provided by (11-8) and (11-10). We will now consider in detail the case of the 12th Lie functor. We will need to introduce additional techniques in order to achieve a complete understanding of the derived functors of $\mathscr{L}^{12}$ and of the differentials in the spectral sequence (11-6) within the range $q \leq 14$.

First observe that only the functors $J^{6} J^{2}, J^{3} J^{2} J^{2}, J^{2} J^{3} J^{2}, J^{4} J^{2} \otimes J^{2} J^{2}$ may give any contribution to the 3-torsion in $L_{q} \mathscr{L}^{12}(A, 1)$ in degrees $q \leq 14$. By (4-3) and (8-14), the derived functors of $J^{3} J^{2} J^{2}$ and $J^{4} J^{2} \otimes J^{2} J^{2}$ are all 2-torsion groups for $A=\mathbb{Z}$. It follows that 3-torsion in $L_{q} \mathscr{L}^{12}(\mathbb{Z}, 1)$ within our range can only occur in degrees $q=10,11$. In fact we will now show that while $J^{6} J^{2}(\mathbb{Z}, 1)=K\left(\mathbb{Z}_{6}, 11\right)$ 
by (10-8), and so could in principle contribute to the 3 -torsion of $L_{11} \mathscr{L}^{12}(\mathbb{Z}, 1)$, this is not in fact the case:

Proposition 11.1 The groups ${ }_{(3)} L_{q} \mathscr{L}^{12}(\mathbb{Z}, 1)=0$ are trivial for all $q \geq 2$.

Proof By (11-7), we may think of the Curtis decomposition of $\mathscr{L}^{12}(\mathbb{Z}, 1)$ as reducing to a short exact sequence

$$
0 \longrightarrow J^{2} J^{3} J^{2}(\mathbb{Z}, 1) \longrightarrow \mathscr{L}^{12}(\mathbb{Z}, 1) \longrightarrow J^{6} J^{2}(\mathbb{Z}, 1) \longrightarrow 0
$$

when only 3 -torsion is considered. This induces following commutative diagram of finite groups with exact horizontal lines, and boundary maps ${ }_{3} \eta_{11}$ :
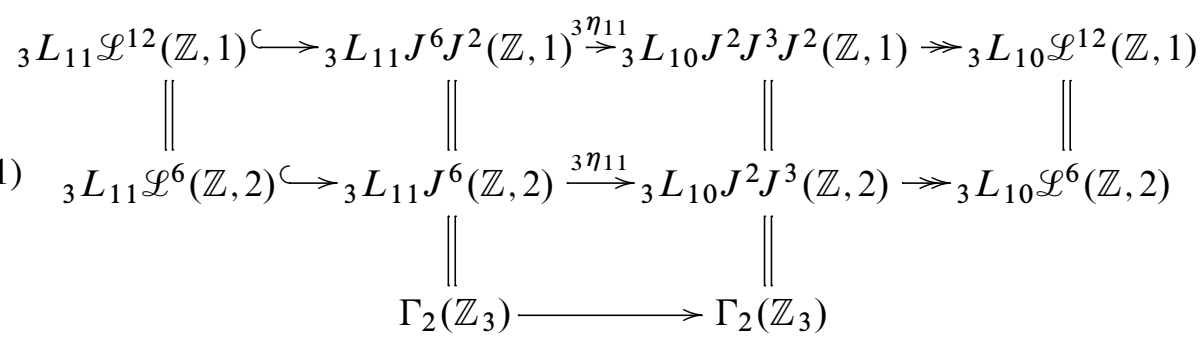

In this diagram, the value of ${ }_{3} L_{11} J^{6}(\mathbb{Z}, 2)$ was determined by (10-8) and that of ${ }_{3} L_{10} J^{2} J^{3} J^{2}(\mathbb{Z}, 1)$ follows from (11-9) and (4-3).

Let us now consider the Curtis spectral sequence (11-4) for the space $K:=K(A, n)$ for some abelian group $A$ :

$$
E_{r, q}^{1}=L_{q+1} \mathscr{L}^{r}(\widetilde{\mathbb{Z}} K(A, n),-1) \Longrightarrow \pi_{q}(K(A, n-1))
$$

We will now look at this in more detail for $A=\mathbb{Z}$ :

$$
E_{r, q}^{1}=L_{q+1} \mathscr{L}^{r}(\widetilde{\mathbb{Z}} K(\mathbb{Z}, n),-1) \Longrightarrow \pi_{q}(K(\mathbb{Z}, n-1))
$$

By Dold's theorem [18, Theorem 5.1], we may replace the expression $\mathbb{Z} K(\mathbb{Z}, n)$ in the initial term of (11-13) by $\bigoplus_{i} K\left(\widetilde{H}_{i+1}(\mathbb{Z}, n), i\right)$ so that the spectral sequence becomes

$$
E_{r, q}^{1}=\pi_{q} \mathscr{L}^{r}\left(\bigoplus_{i} K\left(\tilde{H}_{i+1}(\mathbb{Z}, n), i\right)\right) \Longrightarrow \mathbb{Z}[n-1]
$$

In particular,

$$
E_{1, q}^{1}=\tilde{H}_{q+1}(K(\mathbb{Z}, n)) .
$$

We now consider the case $n=3$. The low-degree (3)-torsion integral homology groups of $K(\mathbb{Z}, 3)$ are well-known by Cartan [11] and Decker [17], in fact the only nontrivial generators for such groups are the fundamental class $i_{3}$ in degree 3 , the degree 7 
suspension of element $\gamma_{3}\left(i_{2}\right) \in H_{6}(K(\mathbb{Z}, 2)$ ), and their product in degree 10 (under the multiplication induced by the $H$-space structure of $K(\mathbb{Z}, 3)$ ):

\begin{tabular}{c|cccccccccc}
$n$ & 3 & 4 & 5 & 6 & 7 & 8 & 9 & 10 & 11 & 12 \\
\hline${ }_{(3)} H_{n} K(\mathbb{Z}, 3)$ & $\mathbb{Z}$ & 0 & 0 & 0 & $\mathbb{Z} / 3$ & 0 & 0 & $\mathbb{Z} / 3$ & 0 & 0
\end{tabular}

When $n=3$, Dold's theorem also allows us to (non-functorially) compute the other initial terms in (11-14), since within the range of values of $q \leq 11$ we may replace the expression $\widetilde{\mathbb{Z}} K(\mathbb{Z}, 3)$ by the product of Eilenberg-Mac Lane spaces $K(\mathbb{Z}, 2) \oplus$ $K(\mathbb{Z} / 3,6) \oplus K(\mathbb{Z} / 3,9)$. For $r=2$ we must therefore compute the homotopy of the induced $L J^{2}(K(\mathbb{Z}, 2) \oplus K(\mathbb{Z} / 3,6) \oplus K(\mathbb{Z} / 3,9))$. No 3-torsion in the homotopy is provided by the functor $J^{2}$ applied to any of the three summands, so the only nontrivial terms are those coming from the cross-effect terms $\mathbb{Z}[2] \otimes \mathbb{Z} / 3[6]$ and $\mathbb{Z}[2] \otimes \mathbb{Z} / 3[9]$, in other words copies of $\mathbb{Z} / 3$ in degrees 8 and 11 respectively.

Similarly, in looking for the 3-torsion of the $r=3$ initial terms of (11-13) within our range of values $q \leq 11$, we need only consider the homotopy of $L J^{3}(K(\mathbb{Z}, 2) \oplus$ $K(\mathbb{Z} / 3,6))$. Let us record here the functorial form of (8-16), for all $n$ and a more restricted range of values of $k$, as extracted from Theorem 8.2:

Lemma 11.2 For any abelian group $A$, and integer $n>4$,

$$
{ }_{3} L_{n+k} J^{3}(A, n)= \begin{cases}A \otimes \mathbb{Z}_{3} & k=3,7 \\ \operatorname{Tor}(A, \mathbb{Z} / 3) & k=4,8 \\ 0 & k=2,5,6 .\end{cases}
$$

It follows that the summand $L J^{3}(\mathbb{Z}, 2)$ contributes a term $\mathbb{Z} / 3$ in degree 5 to the 3 -torsion of $E_{3, q}^{1}$, while the summand $L J^{3}(\mathbb{Z} / 3,6)$ contributes a pair of terms $\mathbb{Z} / 3$ in degrees 9 and 10. In addition, since the second third cross-effect of the functor $J^{3}$ is the functor

$$
J_{[2]}^{3}(A \mid B) \simeq(A \otimes B \otimes A) \oplus(A \otimes B \otimes B),
$$

it contributes an additional term $\mathbb{Z}[2] \otimes \mathbb{Z} / 3[6] \otimes \mathbb{Z}[2]$ to the homotopy group of $L J^{3}(K(\mathbb{Z}, 2) \oplus K(\mathbb{Z} / 3,6))$ in degree 10 , in other words a second factor $\mathbb{Z} / 3$ to the initial term $E_{3,10}^{1}$ of (11-14).

There is no contribution to the 3-torsion component of the initial terms of the spectral sequence (11-13) for $r=4,5,7,8$ since none of these numbers is a multiple of 3 . If we leave aside the case $r=6$ for the time being, the only initial terms which we still need to consider are those for which $r=9$. In our range $q \leq 11$, the only the summand of $\mathscr{L}^{9}$ which comes into play is $J^{3} J^{3}$ and by (8-16) the homotopy groups of $L J^{3} K(\mathbb{Z} / 3,5)$ contribute a pair of groups $\mathbb{Z} / 3$ to the 3 -torsion of $L \mathscr{L}^{9}(\mathbb{Z}, 2)$ in degrees 8 and 9 . 
We collect in Table 7 the outcome of this discussion of the (3)-torsion components of the initial terms of the spectral sequence (11-13) for $n=3$. Since all the terms in

\begin{tabular}{c|ccccccccc}
$r$ & 1 & 2 & 3 & 4 & 5 & 6 & 7 & 8 & 9 \\
\hline${ }_{\text {(3) }} E_{r, 11}^{1}$ & 0 & $\mathbb{Z} / 3$ & 0 & 0 & 0 & $*$ & 0 & 0 & $*$ \\
${ }_{\text {(3) }} E_{r, 10}^{1}$ & 0 & 0 & $(\mathbb{Z} / 3)^{2}$ & 0 & 0 & ${ }_{3} L_{10} \mathscr{L}^{6}(\mathbb{Z}, 2)$ & 0 & 0 & 0 \\
(3) $^{1} E_{r, 9}^{1}$ & $\mathbb{Z} / 3$ & 0 & $\mathbb{Z} / 3$ & 0 & 0 & 0 & 0 & 0 & $\mathbb{Z} / 3$ \\
${ }_{\text {(3) }} E_{r, 8}^{1}$ & 0 & $\mathbb{Z} / 3$ & 0 & 0 & 0 & 0 & 0 & 0 & $\mathbb{Z} / 3$ \\
${ }_{\text {(3) }} E_{r, 7}^{1}$ & 0 & 0 & 0 & 0 & 0 & 0 & 0 & 0 & 0 \\
(3) $E_{r, 6}^{1}$ & $\mathbb{Z} / 3$ & 0 & 0 & 0 & 0 & 0 & 0 & 0 & 0 \\
(3) $E_{r, 5}^{1}$ & 0 & 0 & $\mathbb{Z} / 3$ & 0 & 0 & 0 & 0 & 0 & 0 \\
(3) $E_{r, 4}^{1}$ & 0 & 0 & 0 & 0 & 0 & 0 & 0 & 0 & 0 \\
(3) $E_{r, 3}^{1}$ & 0 & 0 & 0 & 0 & 0 & 0 & 0 & 0 & 0 \\
(3) $E_{r, 2}^{1}$ & $\mathbb{Z}$ & 0 & 0 & 0 & 0 & 0 & 0 & 0 & 0
\end{tabular}

Table 7: The 3-torsion in the initial terms for the spectral sequence (11-13) when $n=3$

the abutment of this spectral sequence vanish (except for a copy of $\mathbb{Z}$ in degree 2), it follows by examining the possible differentials in the spectral sequence that the term ${ }_{(3)} L_{10} \mathscr{L}^{6}(\mathbb{Z}, 2)$ survives all the way to $E_{6,10}^{\infty}$ and must therefore be trivial. Diagram (11-11) now makes it clear that ${ }_{(3)} L_{11} \mathscr{L}^{12}(\mathbb{Z}, 1)={ }_{(3)} L_{11} \mathscr{L}^{6}(\mathbb{Z}, 2)$ also vanishes. These were the only possibly nonvanishing terms within our range of degrees, so that finally

$$
\text { (3) } L_{q} \mathscr{L}^{12}(\mathbb{Z}, 1)=0, \quad q \leq 14 \text {. }
$$

Remark 11.3 A direct computation shows that the triviality of $L_{10} \mathscr{L}^{6}(\mathbb{Z}, 2)$ is equivalent to the assertion the class in $\mathscr{L}_{S}^{6}(\mathbb{Z}, 1)_{4}$, of the element

$$
\begin{aligned}
\zeta= & \left\{\left\{s_{2} s_{1} s_{0}(a), s_{2} s_{1} s_{0}(a), s_{3} s_{1} s_{0}(a)\right\},\left\{s_{3} s_{2} s_{0}(a), s_{3} s_{2} s_{0}(a), s_{3} s_{2} s_{1}(a)\right\}\right\} \\
& -\left\{\left\{s_{2} s_{1} s_{0}(a), s_{2} s_{1} s_{0}(a), s_{3} s_{2} s_{0}(a)\right\},\left\{s_{3} s_{1} s_{0}(a), s_{3} s_{1} s_{0}(a), s_{3} s_{2} s_{1}(a)\right\}\right\} \\
& +\left\{\left\{s_{3} s_{1} s_{0}(a), s_{3} s_{1} s_{0}(a), s_{3} s_{2} s_{0}(a)\right\},\left\{s_{2} s_{1} s_{0}(a), s_{2} s_{1} s_{0}(a), s_{3} s_{2} s_{1}(a)\right\}\right\}
\end{aligned}
$$

is trivial, where $a$ is a generator of $\mathbb{Z}=\pi_{1}(K(\mathbb{Z}, 1))$. It would be of some interest to find a specific element in $\mathscr{L}_{s}^{6}(\mathbb{Z}, 1)_{5}$ with boundary $\zeta$. 
Let us now return to the spectral sequence (11-6), where we now know that $E_{12, q}^{1}=0$ for all $q \leq 14$. The complete set of values of its initial terms in the range $q \leq 14$ is displayed in Table 8. The values of the various terms in this table are justified as

\begin{tabular}{c|cccccccccc}
$r$ & 6 & 12 & 18 & 24 & 30 & 36 & 42 & 48 & 54 & 162 \\
\hline${ }_{3} L_{14} \mathscr{L}^{r}(\mathbb{Z}, 1)$ & 0 & 0 & 0 & 0 & 0 & 0 & 0 & 0 & 0 & $\mathbb{Z} / 3$ \\
${ }_{3} L_{13} \mathscr{L}^{r}(\mathbb{Z}, 1)$ & 0 & 0 & $\mathbb{Z} / 3$ & 0 & 0 & 0 & 0 & 0 & $\mathbb{Z} / 3$ & 0 \\
${ }_{3} L_{12} \mathscr{L}^{r}(\mathbb{Z}, 1)$ & 0 & 0 & $\mathbb{Z} / 3$ & 0 & 0 & 0 & 0 & 0 & $\mathbb{Z} / 3 \oplus \mathbb{Z} / 3$ & 0 \\
${ }_{3} L_{11} \mathscr{L}^{r}(\mathbb{Z}, 1)$ & 0 & 0 & 0 & 0 & 0 & 0 & 0 & 0 & $\mathbb{Z} / 3$ & 0 \\
${ }_{3} L_{10} \mathscr{L}^{r}(\mathbb{Z}, 1)$ & 0 & 0 & 0 & 0 & 0 & 0 & 0 & 0 & 0 & 0 \\
${ }_{3} L_{9} \mathscr{L}^{r}(\mathbb{Z}, 1)$ & 0 & 0 & $\mathbb{Z} / 3$ & 0 & 0 & 0 & 0 & 0 & 0 & 0 \\
${ }_{3} L_{8} \mathscr{L}^{r}(\mathbb{Z}, 1)$ & 0 & 0 & $\mathbb{Z} / 3$ & 0 & 0 & 0 & 0 & 0 & 0 & 0 \\
${ }_{3} L_{7} \mathscr{L}^{r}(\mathbb{Z}, 1)$ & 0 & 0 & 0 & 0 & 0 & 0 & 0 & 0 & 0 & 0 \\
${ }_{3} L_{6} \mathscr{L}^{r}(\mathbb{Z}, 1)$ & 0 & 0 & 0 & 0 & 0 & 0 & 0 & 0 & 0 & 0 \\
${ }_{3} L_{5} \mathscr{L}^{r}(\mathbb{Z}, 1)$ & $\mathbb{Z} / 3$ & 0 & 0 & 0 & 0 & 0 & 0 & 0 & 0 & 0
\end{tabular}

Table 8: The 3-torsion in the initial terms of the spectral sequence (11-6)

follows. Observe first of all that the vanishing of all terms $E_{12, q}^{1}$ terms implies that there are no nonzero terms $E_{r, q}^{1}$ whenever $r$ is a multiple of 12 . Nontrivial terms with $r=18$ arise by applying the functor $J^{3}$ according to the rule of Lemma 11.2 to the cyclic group ${ }_{3} E_{6,5}^{1}=\mathbb{Z} / 3$, so that they are contributed by derived functors of the summands $J^{3} J^{3} J^{2}$ of $\mathscr{L}^{18}$. Applying one more functor $J^{3}$ to each of the two cyclic groups $E_{18,8}^{1}$ and $E_{18,9}^{1}$ provides us, according to the same rule, with two additional copies of $\mathbb{Z} / 3$ in the columns $r=54$. Finally, a last composition with a $J^{3}$ yields the only nontrivial term in column $r=162$ within our range $q \leq 14$. Our discussion makes it clear that this cyclic group has been contributed by the appropriate derived functor of the summands $J^{3} J^{3} J^{3} J^{3} J^{2}$ of $\mathscr{L}^{162}$.

The following description of the 3 -torsion in $\pi_{i}\left(S^{2}\right)$ in the range $i \leq 11$ is a consequence of this discussion, once one takes into account the possible differentials in the spectral sequence:

$$
{ }_{3} \pi_{i}\left(S^{2}\right)= \begin{cases}\mathbb{Z} / 3 & i=6,9,10 \\ 0 & \text { otherwise }\end{cases}
$$

In addition,

$$
\pi_{i}\left(S^{2}\right) \supseteq \mathbb{Z} / 3, \quad i=13,14
$$


We have recovered in this way by purely algebraic methods certain of Toda's results [46]. In fact, it can be shown by comparing once more the differentials in a spectral sequence for the Moore space (11-18) with those in the corresponding spectral sequence for an Eilenberg-Mac Lane space, and by suspension arguments, that the additional differentials $d_{18,12}^{36}: \mathbb{Z} / 3 \rightarrow \mathbb{Z} / 3$ and $d_{18,13}^{36}: \mathbb{Z} / 3 \rightarrow \mathbb{Z} / 3 \oplus \mathbb{Z} / 3$ in (11-6) are both monomorphisms. In this way, the entire description of the 3-torsion in $\pi_{n}\left(S^{2}\right)$ up to degree 14 may be obtained algebraically.

\subsection{Some homotopy groups of $M(A, 2)$}

We now consider the spectral sequence (11-5) for $n=2$ :

$$
E_{r, q}^{1}=L_{q} \mathscr{L}^{r}(A, 1) \Rightarrow \pi_{q+1} M(A, 2) .
$$

For $r=3$, some initial terms in this spectral sequence were computed in Section 8.2. We will now study the terms $E_{4, q}^{1}=L_{q} \mathscr{L}^{4}(A, 1)$. The short exact sequences (2-9) and (7-10) derive to the horizontal lines of the two following commutative diagrams, while the vertical ones arise from semi-décalage and the computations of the groups $L_{i} \Lambda^{2} \Lambda^{2}(A, 1)$ in Table 3:
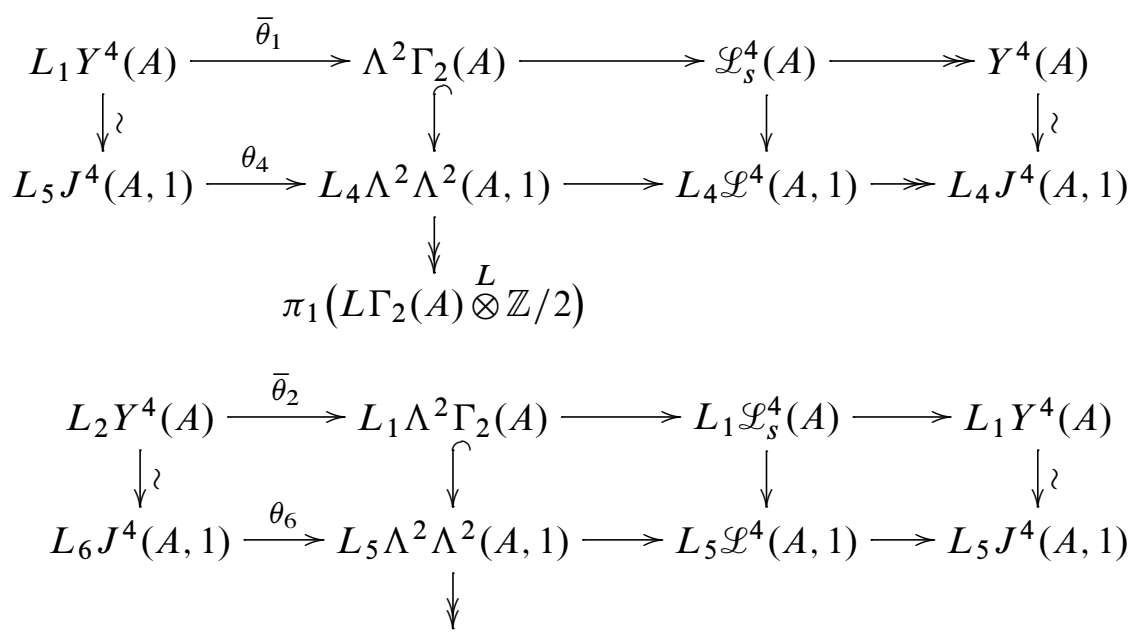

$\operatorname{Tor}\left(R_{2}(A), \mathbb{Z} / 2\right)$

The computation of the $L_{i} \Lambda^{2} \Lambda^{2}(A)$ also implies that there are genuine décalage isomorphisms

$$
L_{i} \mathscr{L}_{s}^{n}(A) \simeq L_{i+n} \mathscr{L}^{n}(A, 1)
$$

for $n=4$ whenever $i>2$. The same is true for $n=5$ and all values of $i$ by comparison of the derived long exact sequences associated to the sequences (7-11) and (7-31). This 
discussion provides the justification ${ }^{3}$ for the values in the columns $r=1,2,3,5$ of Table 9, where the $r$-th column describes, as $q$ varies in the range $1 \leq q \leq 7$, the initial terms $E_{r, q}^{1}$ in the spectral sequence (11-18).

The expressions in the second column of Table 9 follow from (2-11) and from the computation (4-3) for $n=1$, and those in the third column from (8-4). The first summand in the term $E_{4,3}^{1}$ in the fourth column is provided by the subgroup $\Lambda^{2} \Lambda^{2}(A)$ of $\mathscr{L}^{4}(A)$ exhibited in (7-10), once the expression in position $(2,1)$ in Table 3 is taken into account, and its derived versions then occur above it. The second summand in $E_{4,3}^{1}$ arises from the décalage isomorphism (11-20) and a diagram chase in diagram (11-19). Once more, its derived versions are then to be found above it.

We will now show how to find the terms of interest to us in columns 6 and 8 , by the methods of Section 9. Those in the sixth column in degrees $q=4,5$ only depend on the first two summands $J^{3} J^{2}(A)$ and $J^{2} J^{3}(A)$ of $\mathscr{L}^{6}(A)$. The term $J^{2} J^{3}(A)$ in $\mathscr{L}^{6}(A)$ contributes an expression

$$
L_{4}\left(J^{2} J^{3}(A, 1)\right) \simeq L_{4} \Lambda^{2}\left(Y^{3}(A), 3\right) \simeq Y^{3} A \otimes \mathbb{Z} / 2
$$

to $E_{6,4}^{1}$, since $L_{2} \Lambda^{2}(A, 1) \simeq \Gamma_{2} A$ and $L_{5} J^{3}(A, 2) \simeq A \otimes \mathbb{Z} / 3$ (8-14). The same computation provides the corresponding factor in $E_{6,5}^{1}$. Similarly, the term $J^{3} J^{2}(A)$ provides the expression $\Gamma_{2}(A) \otimes \mathbb{Z} / 3$ in $E_{6,5}^{1}$, as $L_{2} \Lambda^{2}(A, 1) \simeq \Gamma_{2} A$ and $L_{5} J^{3}(A, 2) \simeq$ $A \otimes \mathbb{Z} / 3$ (8-14). Finally, the term $E_{8,4}^{1}$ comes from the term $L_{4} J^{2} J^{2} J^{2}(A, 1)$ in $L_{4} \mathscr{L}^{8}(A, 1)$ by the same sort of reasoning. We already know that

$$
L_{3} \Lambda^{2} \Lambda^{2}(A, 1) \simeq \Gamma_{2}(A) \otimes \mathbb{Z} / 2 .
$$

This implies that

$$
L_{4} \Lambda^{2}\left(\Lambda^{2} \Lambda^{2}(A, 1)\right) \simeq L_{4} \Lambda^{2}\left(\Gamma_{2} A \otimes \mathbb{Z} / 2,3\right)
$$

and the result follows since

$$
L_{4} \Lambda^{2}\left(\Gamma_{2} A \otimes \mathbb{Z} / 2,3\right) \simeq L_{6} \operatorname{SP}^{2}\left(\Gamma_{2} A \otimes \mathbb{Z} / 2,4\right) \simeq H_{6}\left(K\left(\Gamma_{2} A \otimes \mathbb{Z} / 2,4\right)\right) \simeq \Gamma_{2} A \otimes \mathbb{Z} / 2,
$$

The last isomorphism follows by a direct calculation, or by reference to the well-know Eilenberg-MacLane functorial stable isomorphism

$$
H_{6}(K(B, 4)) \simeq B \otimes \mathbb{Z} / 2 .
$$

We refer to [40, Section 5.5] for a more complete discussion by one of us of the derived functors of iterates of $\Lambda^{2}$ when $A=\mathbb{Z}$.

\footnotetext{
${ }^{3}$ The validity of some of the higher entries in this table (as well as in Tables 15 and 17) is not verified in the present text, nor are the results in question used here. We refer for the proofs of these assertions, which have been included here for completeness, to the forthcoming [38] .
} 


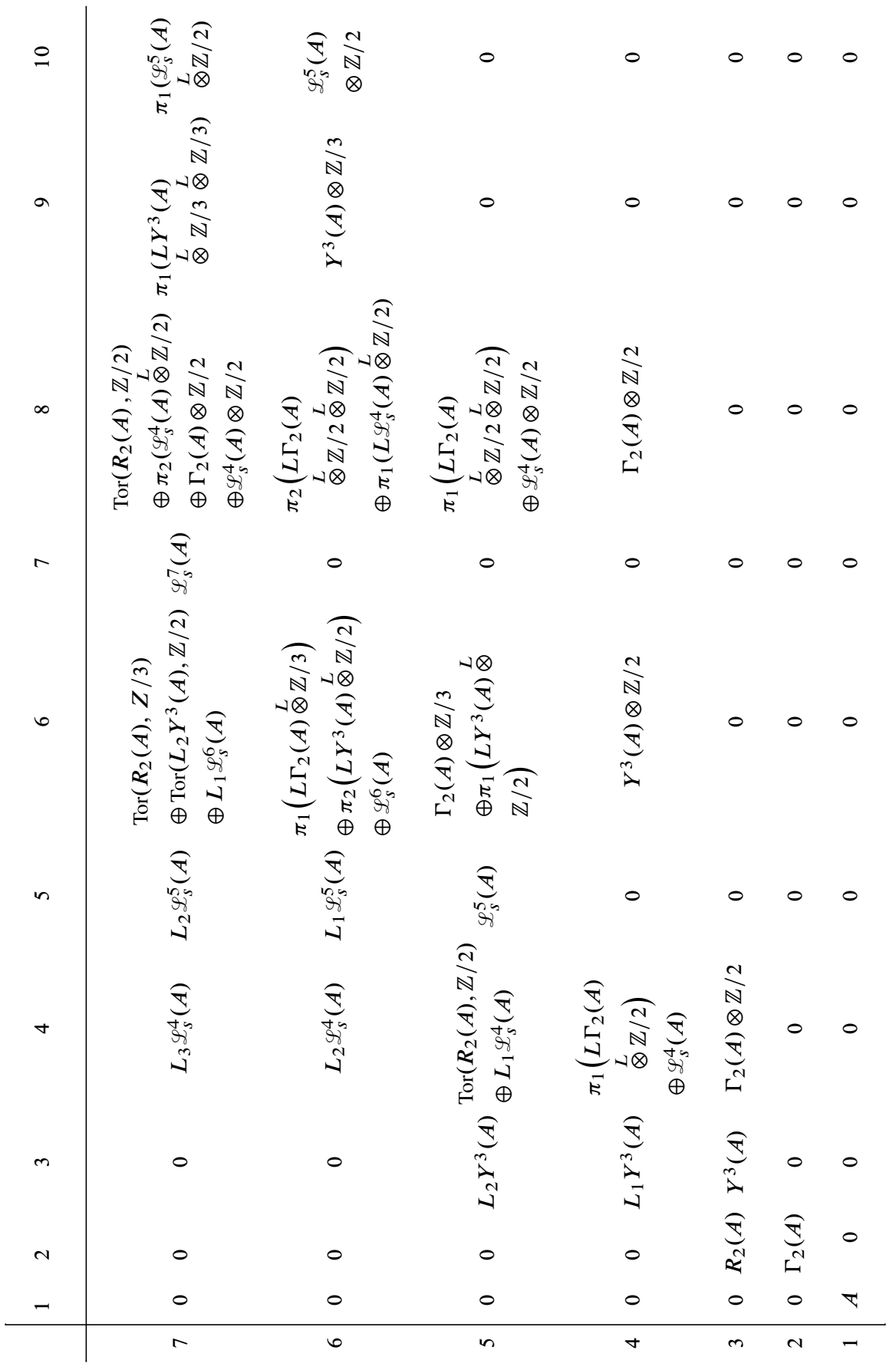

Table 9: The $E_{r, q}^{1}$ terms of the spectral sequence (11-18) 
It is immediate from the line $q=2$ of Table 9 that

$$
\pi_{3}(M(A, 2)) \simeq \Gamma_{2}(A),
$$

a result which essentially goes back to J HC Whitehead's "certain exact sequence" [48], and that in particular a generator of $\Gamma_{2}(\mathbb{Z})$ corresponds to the class of the Hopf map $\eta: S^{3} \longrightarrow S^{2}$. By comparing the spectral sequence (11-18) with the corresponding spectral sequence (11-12) for $n=3$, one verifies that the differential $d_{3,4}^{1}: E_{3,4}^{1} \longrightarrow$ $E_{4,3}^{1}$ in (11-18) is trivial. The line $q=3$ of Table 9 then implies that there is a short exact sequence

$$
0 \rightarrow \mathscr{L}_{s}^{3}(A) \oplus\left(\Gamma_{2}(A) \otimes \mathbb{Z} / 2\right) \rightarrow \pi_{4} M(A, 2) \rightarrow R_{2}(A) \rightarrow 0,
$$

a result already proved in Baues [2] and Baues and Buth [3], where the expression $\mathscr{L}_{S}^{3}(A) \oplus\left(\Gamma_{2}(A) \otimes \mathbb{Z} / 2\right)$ is denoted $\Gamma_{2}^{2}(A)$.

Similarly, the last two terms in the line $q=4$ of our table, together with the factor $\mathscr{L}_{S}^{4}(A)$ from $E_{4,4}^{1}$, regroup to the expression denoted $\Gamma_{2}^{3}(A)$ in [3], while the direct sum of the two remaining terms on the line $q=4$ correspond to the derived functor $L_{1} \Gamma_{2}^{2}(A)$ of the functor $\Gamma_{2}^{2}(A)$ mentioned above. By considering the restriction of the differential $d_{4,5}^{4}: E_{4,5}^{4} \rightarrow E_{8,4}^{4}$ in our table to the factor $\operatorname{Tor}\left(R_{2}(A), \mathbb{Z} / 2\right)$ of $E_{4,5}^{4}$ we therefore recover the description of $\pi_{5} M(A, 2)$ in [3] as a middle term in an exact sequence:

$$
L_{2} \Gamma_{2}^{2}(A) \stackrel{d_{2}}{\rightarrow} \Gamma_{2}^{3}(A) \rightarrow \pi_{5} M(A, 2) \rightarrow L_{1} \Gamma_{2}^{2}(A) \rightarrow 0
$$

where $d_{2}$ is a differential in the spectral sequence from Dreckmann [21] (for a generalized version of this sequence, see Baues and Goerss [4, Theorem 5.1]). We will verify later on in this section (see diagram (11-32)) that this restriction of $d_{4,5}^{4}$ is not zero. This implies that the corresponding differential $d_{2}$ in (11-23) is also nontrivial. This discussion is consistent with the low-dimensional homotopy groups of the Moore space $M(\mathbb{Z} / 2,2)=\Sigma \mathbb{R} P^{2}$ as known from $\mathrm{Wu}[50]$ and displayed in Table 10:

\begin{tabular}{c|cccccr}
$i$ & 2 & 3 & 4 & 5 & 6 & 7 \\
\hline$\pi_{i} M(\mathbb{Z} / 2,2)$ & $\mathbb{Z} / 2$ & $\mathbb{Z} / 4$ & $\mathbb{Z} / 4$ & $(\mathbb{Z} / 2)^{\oplus 3}$ & $(\mathbb{Z} / 2)^{\oplus 5}$ & $(\mathbb{Z} / 2)^{\oplus 2} \oplus(\mathbb{Z} / 4)^{\oplus 2}$ \\
$\oplus \mathbb{Z} / 8$
\end{tabular}

Table 10

Finally, returning to the case $A=\mathbb{Z}$, we also observe in Table 9, in positions $E_{2 p, 2 p-1}^{1}$ with $p$ prime, the early occurrences in $p \pi_{2} p\left(S^{2}\right)$ of Serre's first nontrivial $p$-torsion in the homotopy of $S^{2}$ (see also for this [40, Corollary 5.40 and pages 280-281]). 
Remark 11.4 In the spectral sequence from [21] there are terms $E_{p, q}^{2}=L_{p} \Gamma_{2}^{q}(A)$, where $\Gamma_{2}^{q}(A)$ is the $q$-th term arising from the homotopy operation algebra. In particular, there is a natural homomorphism $\mathscr{L}_{S}^{q+1}(A) \rightarrow \Gamma_{2}^{q}(A)$, where the occurrence of the super-Lie functor $\mathscr{L}_{S}^{q+1}$ is due to Whitehead products, viewed as homotopy operations. It is natural to conjecture that the semi-décalage described in Theorem 10.1 connects the homotopy operation spectral sequence from [21] with the Curtis spectral sequence, with for example the existence of a commutative diagram

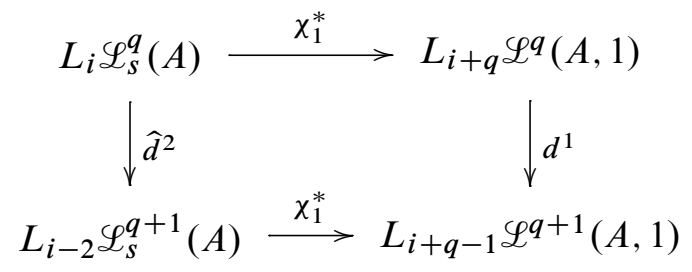

where $\hat{d}^{2}$ is a natural map induced by the second differential in the homotopy operation spectral sequence. The low-dimensional computation given above support this conjectural connection between the two spectral sequences.

\subsection{Some homotopy groups of $M(\mathbb{Z} / p, 2), p \neq 2$}

The next proposition provides us with some information regarding the derived functors of $\mathscr{L}_{S}^{4}(A)$. We begin with the following lemma:

Lemma 11.5 Let $A=\mathbb{Z} / p$ for some prime $p \neq 2$. The natural map $\operatorname{Tor}\left(\Omega_{3}(A), A\right) \rightarrow$ $\Omega_{4}(A)$ is an isomorphism.

Proof By $[10,(5.14)]$, there exists, for any abelian group $A$ and integer $h$, a commutative diagram of abelian groups

$$
\begin{aligned}
& \Gamma_{3}\left({ }_{h} A\right) \otimes{ }_{h} A \longrightarrow \Gamma_{4}\left({ }_{h} A\right) \\
& \downarrow \lambda_{h}^{3} \otimes 1 \quad \downarrow \lambda_{4}^{h} \\
& \operatorname{Tor}\left(\Omega_{3}(A), A\right) \longrightarrow \Omega_{4}(A)
\end{aligned}
$$

where the upper horizontal arrow is induced by the multiplication in the divided power algebra. The arrows $\lambda_{h}^{i}$ provide, where $h$ varies, and the slide relations are taken into account, presentations for the groups $\operatorname{Tor}\left(\Omega_{3}(A), A\right)$ and $\Omega_{4}(A)$ respectively. Let us now suppose that $A$ is cyclic of order $p$, with a chosen generator $a \in A$. In that case the only relevant integer is $h=p$. We know that $\Omega_{3}(\mathbb{Z} / p)=\Omega_{4}(\mathbb{Z} / p)=\mathbb{Z} / p$ so that 
the lower horizontal map in (11-24) is a homomorphism $\mathbb{Z} / p \rightarrow \mathbb{Z} / p$. Let us show that this morphism is nontrivial. The image of $\gamma_{3}(a) \otimes a$ in $\Omega_{4}(\mathbb{Z} / p)$ is

$$
\gamma_{3}(a) \otimes a \mapsto 4 \gamma_{4}(a) \mapsto 4 \omega_{4}^{p}(a)
$$

and $4 \omega_{4}^{p}(a) \neq 0$ since $p \neq 2$.

Proposition 11.6 For any integer $i \geq 0$ and any odd prime $p$, one then has

$$
L_{i} \mathscr{L}_{s}^{4}(\mathbb{Z} / p)= \begin{cases}\mathbb{Z} / p & i=1,2, \\ 0 & i \neq 1,2 .\end{cases}
$$

Proof It follows from definition that $\mathscr{L}_{S}^{4}(A)=0$ for every cyclic group $A$. By (7-30), the sequence

$$
\begin{aligned}
0 \rightarrow L_{3} & \Lambda^{2} \Gamma_{2}(A) \rightarrow L_{3} \mathscr{L}_{s}^{4}(A) \rightarrow L_{3} Y^{4}(A) \rightarrow L_{2} \Lambda^{2} \Gamma_{2}(A) \\
& \rightarrow L_{2} \mathscr{L}_{s}^{4}(A) \rightarrow L_{2} Y^{4}(A) \stackrel{\partial}{\rightarrow} L_{1} \Lambda^{2} \Gamma_{2}(A) \rightarrow L_{1} \mathscr{L}_{s}^{4}(A) \rightarrow L_{1} Y^{4}(A)
\end{aligned}
$$

is exact. By (4-4), $L \Gamma_{2}(\mathbb{Z} / p)=K(\mathbb{Z} / p, 0)$ for $p$ odd, so that

$$
L_{i} \Lambda^{2} \Gamma_{2}(\mathbb{Z} / p)= \begin{cases}\mathbb{Z} / p & i=1, \\ 0 & i \neq 1 .\end{cases}
$$

In particular, the right-hand arrow in (11-25) is surjective. The definition of $Y^{4}$ implies that there is a long exact sequence

$$
\begin{aligned}
L_{2} Y^{4}(A) \rightarrow \pi_{2}\left(L \Lambda^{3}(A) \stackrel{L}{\otimes} A\right) \rightarrow L_{2} \Lambda^{4}(A) & \\
& \rightarrow L_{1} Y^{4}(A) \rightarrow \pi_{1}\left(L \Lambda^{3}(A) \stackrel{L}{\otimes} A\right) \rightarrow L_{1} \Lambda^{4}(A)
\end{aligned}
$$

and an isomorphism

$$
L_{3} Y^{4}(A) \simeq \operatorname{ker}\left\{\operatorname{Tor}\left(\Omega_{3}(A), A\right) \rightarrow \Omega_{4}(A)\right\} .
$$

Lemma 11.5 asserts that the group $L_{3} Y^{4}(\mathbb{Z} / p)$ is trivial, and we know by (2-21) and (2-25) that

$$
L_{i} \Lambda^{4}(\mathbb{Z} / p)= \begin{cases}\mathbb{Z} / p & i=3, \\ 0 & i \neq 3 .\end{cases}
$$

The exactness of the sequence (11-25) then implies that

$$
L_{i} Y^{4}(\mathbb{Z} / p)= \begin{cases}\mathbb{Z} / p & i=2, \\ 0 & i \neq 2 .\end{cases}
$$


We will now show that the boundary map (11-25)

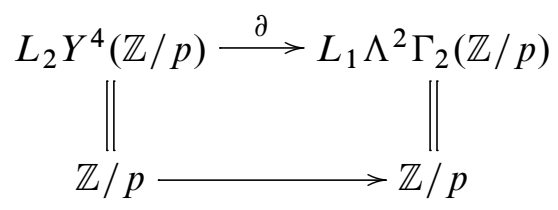

is trivial. Consider first the case $p \neq 2,3$. One then has the following values for the homology groups $H_{*} K(\mathbb{Z} / p, 2)$ :

\begin{tabular}{c|cccccccc}
$n$ & 2 & 3 & 4 & 5 & 6 & 7 & 8 & 9 \\
\hline$H_{n} K(\mathbb{Z} / p, 2)$ & $\mathbb{Z} / p$ & 0 & $\mathbb{Z} / p$ & 0 & $\mathbb{Z} / p$ & 0 & $\mathbb{Z} / p$ & 0
\end{tabular}

The analogue of the spectral sequence (11-13) for $K=K(\mathbb{Z} / p, 2)$ is

$$
E_{r, q}^{1}=L_{q+1} \mathscr{L}^{r}(\widetilde{\mathbb{Z}} K(\mathbb{Z} / p, 2),-1) \Longrightarrow \pi_{q}(K(\mathbb{Z} / p, 1))
$$

Reasoning as in the proof of Proposition 11.1, we find that the initial terms in this spectral sequence are as in Table 11.

\begin{tabular}{c|cccccccccc}
$q$ & $E_{1, q}^{1}$ & $E_{2, q}^{1}$ & $E_{3, q}^{1}$ & $E_{4, q}^{1}$ & $E_{5, q}^{1}$ & $E_{6, q}^{1}$ & $E_{7, q}^{1}$ & $E_{8, q}^{1}$ & $E_{9, q}^{1}$ & $E_{10, q}^{1}$ \\
\hline 8 & 0 & $*$ & $*$ & $*$ & $*$ & $*$ & $*$ & $*$ & $*$ & $*$ \\
7 & $\mathbb{Z} / p$ & $\mathbb{Z} / p$ & $*$ & $*$ & $*$ & $*$ & $*$ & $*$ & $*$ & $*$ \\
6 & 0 & $\mathbb{Z} / p^{2}$ & $\mathbb{Z} / p^{2}$ & $*$ & $*$ & $*$ & $*$ & $*$ & $*$ & $*$ \\
5 & $\mathbb{Z} / p$ & $\mathbb{Z} / p$ & $\mathbb{Z} / p$ & $L_{1} \mathscr{L}_{s}^{4}(\mathbb{Z} / p)$ & 0 & 0 & 0 & 0 & 0 & 0 \\
4 & 0 & $\mathbb{Z} / p$ & $\mathbb{Z} / p$ & 0 & 0 & 0 & 0 & 0 & 0 & 0 \\
3 & $\mathbb{Z} / p$ & 0 & 0 & 0 & 0 & 0 & 0 & 0 & 0 & 0 \\
2 & 0 & $\mathbb{Z} / p$ & 0 & 0 & 0 & 0 & 0 & 0 & 0 & 0 \\
1 & $\mathbb{Z} / p$ & 0 & 0 & 0 & 0 & 0 & 0 & 0 & 0 & 0
\end{tabular}

Table 11: Some $E^{1}$ terms in the spectral sequence (11-29) for $p \neq 2,3$

This spectral sequence converges to the graded group $\mathbb{Z} / p[1]$. Let us suppose that $L_{1} \mathscr{L}_{s}^{4}(\mathbb{Z} / p)=0$. In that case $E_{2,6}^{\infty} \oplus E_{3,6}^{\infty} \neq 0$ and this contradicts the fact that homotopy groups $\pi_{i} K(\mathbb{Z} / p, 1)$ are trivial for $i \geq 2$. It follows by (11-26) and (11-27) that $L_{1} \mathscr{L}_{s}^{4}(\mathbb{Z} / p)=\mathbb{Z} / p$ and the map (11-28) is the zero map. The description of all the derived functors of $\mathscr{L}_{s}^{4}(\mathbb{Z} / p)$ for $p \neq 2,3$ now follows from the exact sequence (11-25). 
We now consider the $p=3$ case. We have the following description of the low degree homology of $K(\mathbb{Z} / 3,2)$ :

\begin{tabular}{c|cccccccc}
$n$ & 2 & 3 & 4 & 5 & 6 & 7 & 8 & 9 \\
\hline$H_{n} K(\mathbb{Z} / 3,2)$ & $\mathbb{Z} / 3$ & 0 & $\mathbb{Z} / 3$ & 0 & $\mathbb{Z} / 9$ & $\mathbb{Z} / 3$ & $\mathbb{Z} / 3$ & $\mathbb{Z} / 3$
\end{tabular}

The initial terms in the spectral sequence (11-29) for $p=3$ are given in Table 12.

\begin{tabular}{c|cccccccccc}
$q$ & $E_{1, q}^{1}$ & $E_{2, q}^{1}$ & $E_{3, q}^{1}$ & $E_{4, q}^{1}$ & $E_{5, q}^{1}$ & $E_{6, q}^{1}$ & $E_{7, q}^{1}$ & $E_{8, q}^{1}$ & $E_{9, q}^{1}$ & $E_{10, q}^{1}$ \\
\hline 8 & $\mathbb{Z} / 3$ & $*$ & $*$ & $*$ & $*$ & $*$ & $*$ & $*$ & $*$ & $*$ \\
7 & $\mathbb{Z} / 3$ & $\mathbb{Z} / 3^{2}$ & $*$ & $*$ & $*$ & $*$ & $*$ & $*$ & $*$ & $*$ \\
6 & $\mathbb{Z} / 3$ & $\mathbb{Z} / 3^{2}$ & $\mathbb{Z} / 3^{3}$ & $*$ & $*$ & $*$ & $*$ & $*$ & $*$ & $*$ \\
5 & $\mathbb{Z} / 9$ & $\mathbb{Z} / 3$ & $\mathbb{Z} / 3^{2}$ & $L_{1} \mathscr{L}_{s}^{4}(\mathbb{Z} / 3)$ & 0 & $\mathbb{Z} / 3$ & 0 & 0 & 0 & 0 \\
4 & 0 & $\mathbb{Z} / 3$ & $\mathbb{Z} / 9$ & 0 & 0 & 0 & 0 & 0 & 0 & 0 \\
3 & $\mathbb{Z} / 3$ & 0 & 0 & 0 & 0 & 0 & 0 & 0 & 0 & 0 \\
2 & 0 & $\mathbb{Z} / 3$ & 0 & 0 & 0 & 0 & 0 & 0 & 0 & 0 \\
1 & $\mathbb{Z} / 3$ & 0 & 0 & 0 & 0 & 0 & 0 & 0 & 0 & 0
\end{tabular}

Table 12: Some $E_{r, q}^{1}$ terms in the spectral sequence (11-29) for $p=3$

We will now prove that the expression $L_{1} \mathscr{L}_{S}^{4}(\mathbb{Z} / 3)$ is equal to $\mathbb{Z} / 3$. For any abelian group $A$, the differentials $d_{1,7}^{1}$ and $d_{1,8}^{1}$ in the corresponding spectral sequence (11-12) for $n=2$ have the property that the following natural diagrams are commutative
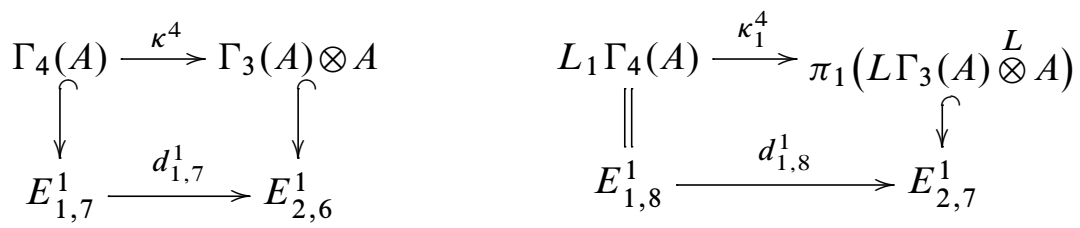

where $\kappa^{4}$ is the homomorphism in the Koszul complex $\operatorname{Kos}^{4}\left(A \stackrel{1_{A}}{\rightarrow} A\right)(2-30)$ and $\kappa_{1}^{4}$ its first derived analog. This implies, in the case $A=\mathbb{Z} / 3$, that the differentials $d_{1,7}^{1}$ and $d_{1,8}^{1}$ are monomorphisms. The assumption $L_{1} \mathscr{L}_{s}^{4}(\mathbb{Z} / 3)=0$, implies that $E_{3,6}^{\infty} \neq 0$ and this contradicts the triviality of the sixth homotopy group of $K(\mathbb{Z} / 3,1)$.

Remark 11.7 For $p=2$ the description of $L_{i} \mathscr{L}_{s}^{4}(\mathbb{Z} / p)$ is also more complicated. For example, the group $L_{2} \mathscr{L}_{s}^{4}(\mathbb{Z} / 2)$ contains nontrivial 4 -torsion elements. In the simplicial language, a generator of the 4 -torsion subgroup is provided by the following 
element:

$$
\begin{aligned}
\left\{s_{0} a_{1}, s_{1} a_{1}, s_{0} a_{1}, s_{1} a_{1}\right\} & -\left\{s_{1} a_{1}, s_{1} s_{0} a_{0}, s_{0} a_{1}, s_{0} a_{1}\right\} \\
+ & \left\{s_{0} a_{1}, s_{1} s_{0} a_{0}, s_{1} a_{0}, s_{1} a_{0}\right\}+\left\{\left\{s_{1} a_{1}, s_{1} s_{0} a_{0}\right\},\left\{s_{0} a_{1}, s_{1} s_{0} a_{0}\right\}\right\}
\end{aligned}
$$

This 4-torsion element corresponds to twice the 8-torsion element of Cohen and $\mathrm{Wu}$ [13] and $\mathrm{Wu}$ [50, Appendix A], which lives in the last summand of $\pi_{7} \Sigma \mathbb{R} P^{2}=$ $\pi_{7} M(\mathbb{Z} / 2,2)$ (see Table 10). We will not discuss this computation, since it involves more elaborate techniques than those described here.

After these preliminaries regarding the derived functors of $\mathscr{L}_{S}^{4}$, let us begin our computations of the homotopy of the spaces $M(\mathbb{Z} / p, 2)$ with the case $p=3$. By Remark 8.1 , we know that

$$
L_{i} \mathscr{L}^{3}(\mathbb{Z} / 3,1)= \begin{cases}\mathbb{Z} / 9 & i=4, \\ \mathbb{Z} / 3 & i=5, \\ 0 & i \neq 4,5\end{cases}
$$

The computation of the derived functors $L_{q} \mathscr{L}^{r}(\mathbb{Z} / 3,1)$ for $q<7$ follows easily from the Curtis decomposition of $\mathscr{L}^{r}$ and the known values of the derived functors of $\mathscr{L}^{3}(\mathbb{Z} / 3,1)$. We display the result in Table 13 .

\begin{tabular}{c|cccccccccc}
$q$ & $E_{1, q}^{1}$ & $E_{2, q}^{1}$ & $E_{3, q}^{1}$ & $E_{4, q}^{1}$ & $E_{5, q}^{1}$ & $E_{6, q}^{1}$ & $E_{7, q}^{1}$ & $E_{8, q}^{1}$ & $E_{9, q}^{1}$ & $E_{10, q}^{1}$ \\
\hline 6 & 0 & 0 & 0 & $\mathbb{Z} / 3$ & $\mathbb{Z} / 3$ & $\mathbb{Z} / 3$ & 0 & 0 & 0 & 0 \\
5 & 0 & 0 & $\mathbb{Z} / 3$ & $\mathbb{Z} / 3$ & 0 & $\mathbb{Z} / 3$ & 0 & 0 & 0 & 0 \\
4 & 0 & 0 & $\mathbb{Z} / 9$ & 0 & 0 & 0 & 0 & 0 & 0 & 0 \\
3 & 0 & 0 & 0 & 0 & 0 & 0 & 0 & 0 & 0 & 0 \\
2 & 0 & $\mathbb{Z} / 3$ & 0 & 0 & 0 & 0 & 0 & 0 & 0 & 0 \\
1 & $\mathbb{Z} / 3$ & 0 & 0 & 0 & 0 & 0 & 0 & 0 & 0 & 0
\end{tabular}

Table 13: The $E^{1}$-term of the spectral sequence (11-5) for $A=\mathbb{Z} / 3$ and $n=2$

The differentials $d_{5,6}^{1}: \mathbb{Z} / 3 \rightarrow \mathbb{Z} / 3$ and $d_{4,6}^{2}: \mathbb{Z} / 3 \rightarrow \mathbb{Z} / 3$ in this table are trivial, as follows from the comparison between the Curtis spectral sequences for $K=M(\mathbb{Z} / 3,2)$ and $K=K(\mathbb{Z} / 3,2)$ and from the structure of Table 13. The assumption that either $d_{5,6}^{1}$ or $d_{4,6}^{2}$ is an isomorphism would produce a nontrivial term $E_{3,6}^{\infty}$ in the spectral sequence whose initial terms were given in Table 12. Looking at the horizontal lines in 
Table 13, we now see that

$$
\begin{aligned}
\pi_{2} M(\mathbb{Z} / 3,2) & =\mathbb{Z} / 3 \\
\pi_{3} M(\mathbb{Z} / 3,2) & =\mathbb{Z} / 3 \\
\pi_{4} M(\mathbb{Z} / 3,2) & =0 \\
\pi_{5} M(\mathbb{Z} / 3,2) & =\mathbb{Z} / 9 \\
\left|\pi_{6} M(\mathbb{Z} / 3,2)\right| & =27 .
\end{aligned}
$$

Observe that we have in particular exhibited here the cyclic group of order 9 of Neisendorfer [42] and Leibowitz [33] mentioned in the introduction.

The homotopy groups of the spaces $M(\mathbb{Z} / p, 2)$ for a prime $p \neq 2,3$, are simpler to describe. In that case, the initial terms of the spectral sequence (11-5) are displayed in Table 14.

\begin{tabular}{c|cccccccccc}
$q$ & $E_{1, q}^{1}$ & $E_{2, q}^{1}$ & $E_{3, q}^{1}$ & $E_{4, q}^{1}$ & $E_{5, q}^{1}$ & $E_{6, q}^{1}$ & $E_{7, q}^{1}$ & $E_{8, q}^{1}$ & $E_{9, q}^{1}$ & $E_{10, q}^{1}$ \\
\hline 6 & 0 & 0 & 0 & $\mathbb{Z} / p$ & $\mathbb{Z} / p$ & 0 & 0 & 0 & 0 & 0 \\
5 & 0 & 0 & 0 & $\mathbb{Z} / p$ & 0 & 0 & 0 & 0 & 0 & 0 \\
4 & 0 & 0 & $\mathbb{Z} / p$ & 0 & 0 & 0 & 0 & 0 & 0 & 0 \\
3 & 0 & 0 & 0 & 0 & 0 & 0 & 0 & 0 & 0 & 0 \\
2 & 0 & $\mathbb{Z} / p$ & 0 & 0 & 0 & 0 & 0 & 0 & 0 & 0 \\
1 & $\mathbb{Z} / p$ & 0 & 0 & 0 & 0 & 0 & 0 & 0 & 0 & 0
\end{tabular}

Table 14: The $E^{1}$-term of the spectral sequence (11-5) for $A=\mathbb{Z} / p$ when $p \neq 2,3$ and $n=2$

In particular, the derived functors $L_{i} \mathscr{L}^{3}(\mathbb{Z} / p, 1)$ are simpler for these values of $p$, which explains the difference between the third columns in Table 13 and Table 14. Note also that the $p$-torsion in $L_{4} \mathscr{L}^{3}(\mathbb{Z} / p, 1)$ comes from the term $\operatorname{ker}\left\{\Omega_{2}(\mathbb{Z} / p) \otimes \mathbb{Z} / p \rightarrow\right.$ $\left.L_{1} \Lambda^{3}(\mathbb{Z} / p)\right\}$ in (8-8). We obtain in particular

$$
\begin{aligned}
\pi_{6} M(\mathbb{Z} / p, 2) & =\mathbb{Z} / p \\
\left|\pi_{7} M(\mathbb{Z} / p, 2)\right| & =p^{2} .
\end{aligned}
$$

\subsection{Some homotopy groups of $M(A, 3)$}

Consider the spectral sequence (11-5) for $n=3$ :

$$
E_{r, q}^{1}=L_{q} \mathscr{L}^{r}(A, 2) \Rightarrow \pi_{q+1} M(A, 3) .
$$


The initial terms of this spectral sequence are obtained as in the spectral sequence (11-18) from the Curtis decomposition of Lie functors and the computation of the derived functors of its graded components. In addition, the occurrence of a summand $\mathscr{L}^{3}(A)$ in $E_{3,6}^{1}$ follows from the composition of the maps (10-2). These initial terms are given in Table 15 (the functor $\lambda^{2}$ was defined by (4-1)).

As a result we have a natural isomorphism

$$
\pi_{4} M(A, 3) \simeq A \otimes \mathbb{Z} / 2,
$$

which is simply the suspended version of the isomorphism (11-21), as well as the following natural short exact sequence:

$$
0 \rightarrow A \otimes \mathbb{Z} / 2 \rightarrow \pi_{5} M(A, 3) \rightarrow \lambda^{2}(A) \rightarrow 0 .
$$

However, the latter is not split, since it is known for example that $\pi_{5} M(\mathbb{Z} / 2,3)=\mathbb{Z} / 4$.

The differential $d_{3,6}^{1}: E_{3,6}^{1} \rightarrow E_{4,5}^{1}$ in Table 15 is trivial, as can be seen by reduction to the case of $A$ free abelian of finite rank, and a comparison of the rank of $E_{3,6}^{1}$ with that of the homotopy group of the corresponding wedge of spheres $S^{3}$, as computed by the Hilton-Milnor theorem (see Curtis [16, Theorem 4.21]). On the other hand, the differential $d_{4,6}^{4}: E_{4,6}^{4} \rightarrow E_{8,5}^{4}$ can be nontrivial. It is an isomorphism for $A=\mathbb{Z} / 2$, as follows from the known description of the groups $\pi_{i}(M(\mathbb{Z} / 2,3))=\pi_{i}\left(\Sigma^{2} \mathbb{R} P^{2}\right)$ for small values of $i$ :

\begin{tabular}{c|cccccc}
$i$ & 3 & 4 & 5 & 6 & 7 & 8 \\
\hline$\pi_{i} M(\mathbb{Z} / 2,3)$ & $\mathbb{Z} / 2$ & $\mathbb{Z} / 2$ & $\mathbb{Z} / 4$ & $\mathbb{Z} / 4 \oplus \mathbb{Z} / 2$ & $\mathbb{Z} / 2 \oplus \mathbb{Z} / 2$ & $\mathbb{Z} / 2 \oplus \mathbb{Z} / 2$
\end{tabular}

In addition, one can express the differential $d_{4,6}^{4}$ in (11-30) as a suspension by comparing the spectral sequences (11-18) and (11-30). For this, consider the following commutative diagram, in which the vertical arrows are suspension morphisms:

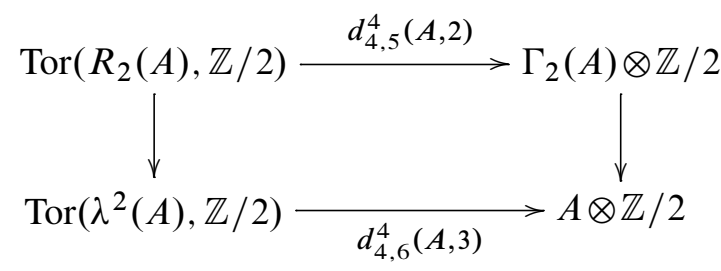

The upper arrow in this diagram is the restriction to the first summand of the differential $d_{4,5}^{4}$ from (11-18), whereas the lower one is the differential $d_{4,6}^{4}$ from (11-30). The suspension maps are isomorphisms for $A=\mathbb{Z} / 2$. Since we know that $d_{4,6}^{4}$ is an isomorphism in that case, so is the differential $d_{4,5}^{4}$ in (11-18). 


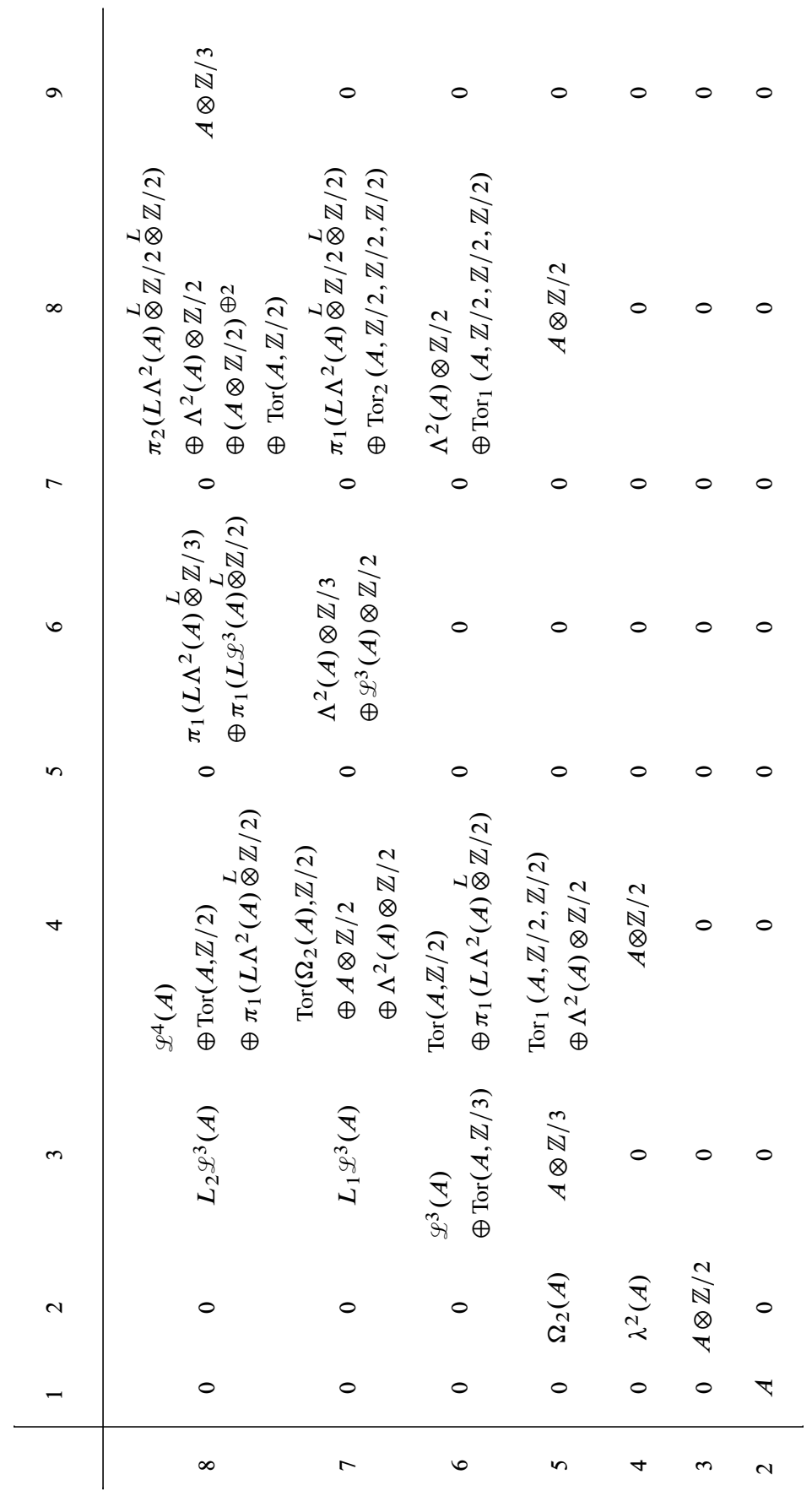

Table 15: The initial terms $E_{p, q}^{1}$ of the spectral sequence (11-30) for $2 \leq q \leq 8$ 
The spectral sequence (11-30) determines in particular a filtration on $\pi_{6} M(A, 3)$, with the following nontrivial associated graded components:

$$
\begin{aligned}
& \operatorname{gr}_{2} \pi_{6} M(A, 3)=\Omega_{2}(A) \\
& \operatorname{gr}_{3} \pi_{6} M(A, 3)=A \otimes \mathbb{Z} / 3 \\
& \operatorname{gr}_{4} \pi_{6} M(A, 3)=\left(\Lambda^{2}(A) \otimes \mathbb{Z} / 2\right) \oplus \operatorname{Tor}_{1}(A, \mathbb{Z} / 2, \mathbb{Z} / 2) \\
& \operatorname{gr}_{8} \pi_{6} M(A, 3)=A \otimes \mathbb{Z} / 2 / \operatorname{im}\left(d_{4,6}^{4}\right)
\end{aligned}
$$

For $A=\mathbb{Z}$, this determines precisely 12 elements in $\pi_{6}\left(S^{3}\right)$, which are the nontrivial elements in the associated graded components $\mathrm{gr}_{2}, \mathrm{gr}_{3}, \mathrm{gr}_{8}$ listed above. Table 15 also implies that there is an epimorphism

$$
\pi_{7} M(A, 3) \rightarrow \mathscr{L}^{3}(A) \oplus \operatorname{Tor}\left(A, \mathbb{Z}_{3}\right) .
$$

As an example of this computation, consider the case $A=\mathbb{Z} / 3$. A simple analysis, with the help of (8-14), gives the description of the initial terms of the corresponding spectral sequence (11-30) in Table 16.

\begin{tabular}{c|cccccccccc}
$q$ & $E_{1, q}^{1}$ & $E_{2, q}^{1}$ & $E_{3, q}^{1}$ & $E_{4, q}^{1}$ & $E_{5, q}^{1}$ & $E_{6, q}^{1}$ & $E_{7, q}^{1}$ & $E_{8, q}^{1}$ & $E_{9, q}^{1}$ & $E_{10, q}^{1}$ \\
\hline 8 & 0 & 0 & 0 & 0 & 0 & $\mathbb{Z} / 3$ & 0 & 0 & $\mathbb{Z} / 3$ & 0 \\
7 & 0 & 0 & $\mathbb{Z} / 3$ & 0 & 0 & 0 & 0 & 0 & 0 & 0 \\
6 & 0 & 0 & $\mathbb{Z} / 3$ & 0 & 0 & 0 & 0 & 0 & 0 & 0 \\
5 & 0 & $\mathbb{Z} / 3$ & $\mathbb{Z} / 3$ & 0 & 0 & 0 & 0 & 0 & 0 & 0 \\
4 & 0 & 0 & 0 & 0 & 0 & 0 & 0 & 0 & 0 & 0 \\
3 & 0 & 0 & 0 & 0 & 0 & 0 & 0 & 0 & 0 & 0 \\
2 & $\mathbb{Z} / 3$ & 0 & 0 & 0 & 0 & 0 & 0 & 0 & 0 & 0
\end{tabular}

Table 16: The initial terms of the spectral sequence (11-30) for $A=\mathbb{Z} / 3$

We conclude that $\pi_{7} M(\mathbb{Z} / 3,3)=\pi_{8} M(\mathbb{Z} / 3,3)=\mathbb{Z} / 3$.

\subsection{Some homotopy groups of $M(A, 4)$}

The spectral sequence (11-5) for $n=4$

$$
E_{r, q}^{1}=L_{q} \mathscr{L}^{r}(A, 3) \Rightarrow \pi_{q+1} M(A, 4)
$$

has initial terms in low dimensions given by Table 17. Observe in particular that the torsion-free expression $\Gamma_{2}(\mathbb{Z})$ which appears in column 2 of Table 17 for $A=\mathbb{Z}$ survives to $E_{2,6}^{\infty}$ since a nontrivial morphism $d_{2,6}^{2}: \Gamma_{2}(\mathbb{Z}) \longrightarrow \mathbb{Z} \otimes \mathbb{Z} / 2$ would contradict 


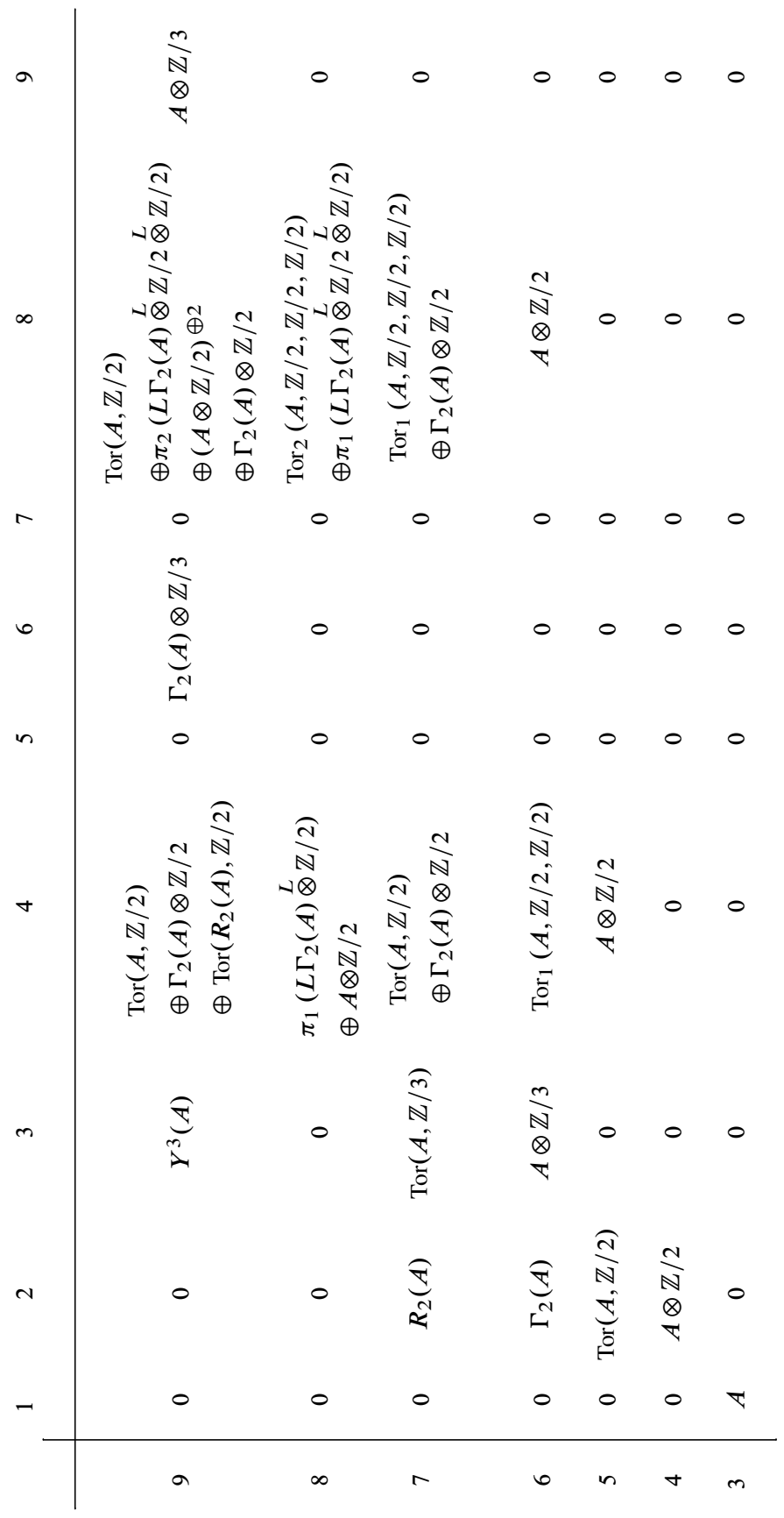

Table 17: The initial terms for $q \leq 9$ in the spectral sequence (11-5) with $n=4$ 
the known nontrivial value of $\pi_{6}\left(S^{4}\right)$. We can therefore recognize in a generator of this group $\Gamma_{2}(\mathbb{Z})$ the class of the generalized Hopf fibration $v: S^{7} \rightarrow S^{4}$.

The suspension homomorphisms $\pi_{4} M(A, 2) \rightarrow \pi_{5} M(A, 3) \rightarrow \pi_{6} M(A, 4)$ can be described in terms of the suspension homomorphisms between the corresponding derived functors of Lie functors. The result, which can be read off from (11-22), (11-31) and Table 17, is expressed by the following commutative diagram (see also Baues [2, VIII Section 3, IX Section 2, XI Section 1]):

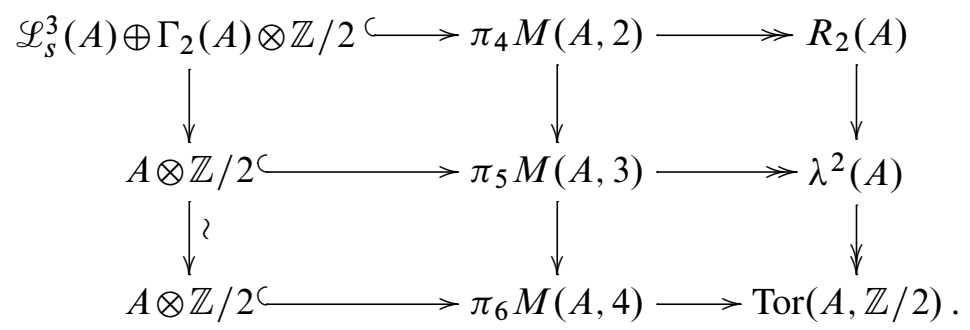

Note that these horizontal short exact sequences are in general not split, since

$$
\pi_{4}(M(\mathbb{Z} / 2,2))=\pi_{5}(M(\mathbb{Z} / 2,3))=\mathbb{Z} / 4 \text {. }
$$

\subsection{Solving the extension problem}

In simple cases one can solve the extension problems with the help of functoriality. For example we have just seen that there is a natural exact sequence

$$
0 \rightarrow A \otimes \mathbb{Z} / 2 \rightarrow \pi_{6} M(A, 4) \rightarrow \operatorname{Tor}(A, \mathbb{Z} / 2) \rightarrow 0 .
$$

For $A=\mathbb{Z} / 4$, this reduces to the sequence

$$
0 \rightarrow \mathbb{Z} / 2 \rightarrow \pi_{6} M(\mathbb{Z} / 4,4) \rightarrow \mathbb{Z} / 2 \rightarrow 0 .
$$

In order to compute the group $\pi_{6} M(\mathbb{Z} / 4,4)$, we must still determine whether this sequence is split. The following simple argument will show that this indeed is the case.

Let $F$ be an endofunctor on the category of abelian groups which can be expressed as a natural extension of functors

$$
0 \rightarrow A \otimes \mathbb{Z} / 2 \rightarrow F(A) \rightarrow \operatorname{Tor}(A, \mathbb{Z} / 2) \rightarrow 0 .
$$

We will now prove that for any such functor $F$ this extension is split. Suppose on the contrary that $F(\mathbb{Z} / 4)=\mathbb{Z} / 4$. In that case the group $F(\mathbb{Z} / 2)$ is isomorphic either 
to $\mathbb{Z} / 4$ or to $\mathbb{Z} / 2 \oplus \mathbb{Z} / 2$. Let us first suppose that $F(\mathbb{Z} / 2)=\mathbb{Z} / 4$ and consider the following diagram, induced by the injection of $\mathbb{Z} / 2$ into $\mathbb{Z} / 4$ :

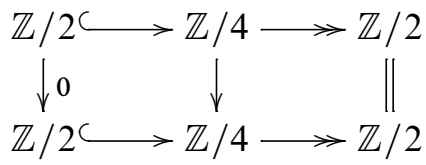

Such a commutative diagram cannot exist since the pushout of any extension by the trivial homomorphism is a trivial extension. If on the other hand we suppose that $F(\mathbb{Z} / 2)=\mathbb{Z} / 2 \oplus \mathbb{Z} / 2$, then the natural projection $\mathbb{Z} / 4 \rightarrow \mathbb{Z} / 2$ induces a commutative diagram

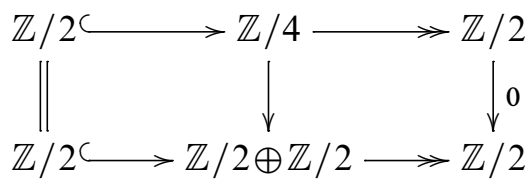

which also cannot exist, since the pullback of any extension by the trivial homomorphism is a trivial extension. This proves that the extension (11-34) is split, and in particular that $\pi_{6} M(\mathbb{Z} / 4,4)=\mathbb{Z} / 2 \oplus \mathbb{Z} / 2$.

\subsection{Some homotopy groups of $M(\mathbb{Z} / 3,5)$}

In this simple example, we will illustrate some lines of reasoning by which we computed certain differentials in Curtis spectral sequences. Consider such a spectral sequence (11-3) for $n=5$, with abutment $M(\mathbb{Z} / 3,5)$ and initial terms $E_{r, q}^{1}=L_{q} \mathscr{L}^{r}(\mathbb{Z} / 3,4)$. One finds in low degree the values given in Table 18.

\begin{tabular}{c|ccccccccc}
$q$ & $E_{1, q}^{1}$ & $E_{2, q}^{1}$ & $E_{3, q}^{1}$ & $E_{4, q}^{1}$ & $E_{5, q}^{1}$ & $E_{6, q}^{1}$ & $E_{7, q}^{1}$ & $E_{8, q}^{1}$ & $E_{9, q}^{1}$ \\
\hline 10 & 0 & 0 & 0 & 0 & 0 & 0 & 0 & 0 & $\mathbb{Z} / 3$ \\
9 & 0 & $\mathbb{Z} / 3$ & 0 & 0 & 0 & 0 & 0 & 0 & 0 \\
8 & 0 & 0 & $\mathbb{Z} / 3$ & 0 & 0 & 0 & 0 & 0 & 0 \\
7 & 0 & 0 & $\mathbb{Z} / 3$ & 0 & 0 & 0 & 0 & 0 & 0 \\
6 & 0 & 0 & 0 & 0 & 0 & 0 & 0 & 0 & 0 \\
5 & 0 & 0 & 0 & 0 & 0 & 0 & 0 & 0 & 0 \\
4 & $\mathbb{Z} / 3$ & 0 & 0 & 0 & 0 & 0 & 0 & 0 & 0
\end{tabular}

Table 18: The initial terms in the spectral sequence (11-5) for $n=5$ and $A=\mathbb{Z} / 3$

We will now provide two separate justifications for the triviality of the differential $d_{2,9}^{1}: \mathbb{Z} / 3 \rightarrow \mathbb{Z} / 3$, both of which were used in more complex situations in the previous 
paragraphs. The first argument goes as follows. The differential $d_{2,9}^{1}$ for $n=5$ and $A=\mathbb{Z} / 3$ lives in the following commutative diagram, in which the notation is the same as in diagram (11-32) (and the vertical arrows are suspension maps):

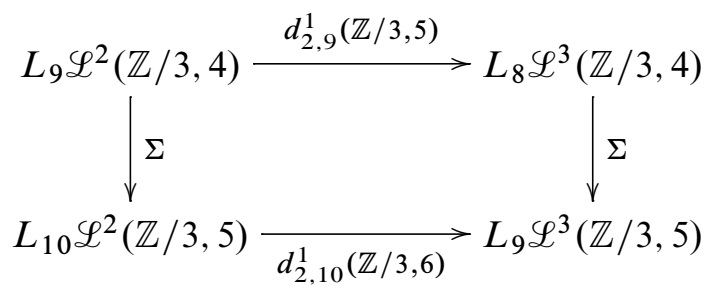

This commutative square is actually of the form

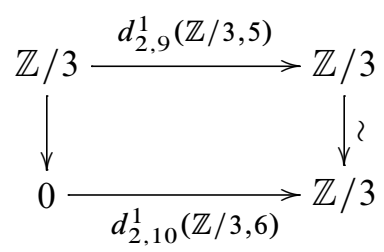

so that the map $d_{2,9}^{1}(\mathbb{Z} / 3,5)$ is trivial. As a consequence

$$
\pi_{9} M(\mathbb{Z} / 3,5)=\pi_{10} M(\mathbb{Z} / 3,5)=\mathbb{Z} / 3 .
$$

Here is the second proof of this assertion. Consider the natural map $M(\mathbb{Z} / 3,5) \rightarrow$ $K(\mathbb{Z} / 3,5)(11-1)$, and the corresponding map between the spectral sequences $(11-5)$ and (11-14) for $n=5$ and $A=\mathbb{Z} / 3$. We now display the low-dimensional homology groups of $K(\mathbb{Z} / 3,5)$ :

\begin{tabular}{c|ccccccc}
$n$ & 5 & 6 & 7 & 8 & 9 & 10 & 11 \\
\hline$H_{n} K(\mathbb{Z} / 3,5)$ & $\mathbb{Z} / 3$ & 0 & 0 & 0 & $\mathbb{Z} / 3$ & $\mathbb{Z} / 3$ & $\mathbb{Z} / 3$
\end{tabular}

The initial terms of the spectral sequence (11-14) for $n=5$ and $A=\mathbb{Z} / 3$ are given in Table 19. In this Table 19, we display within brackets those terms which are in the image of elements from the corresponding spectral sequence (11-5), as given in Table 18. Since the spectral sequence (11-12) converges here to the graded group $\mathbb{Z} / 3$ concentrated in degree 4 , it follows that the map $d_{2,9}^{1}$ is necessarily zero: otherwise, the element $E_{1,10}^{1}=\mathbb{Z} / 3$ would contribute nontrivially to $\pi_{10}(K(\mathbb{Z} / 3,4))$. It now follows that the corresponding map $d_{2,9}^{1}$ in the spectral sequence whose initial terms are displayed in Table 18 is also trivial. We deduce from this that $\pi_{9} M(\mathbb{Z} / 3,5)=$ $\pi_{10} M(\mathbb{Z} / 3,5)=\mathbb{Z} / 3$. 


\begin{tabular}{c|ccccccccc}
$q$ & $E_{1, q}^{1}$ & $E_{2, q}^{1}$ & $E_{3, q}^{1}$ & $E_{4, q}^{1}$ & $E_{5, q}^{1}$ & $E_{6, q}^{1}$ & $E_{7, q}^{1}$ & $E_{8, q}^{1}$ & $E_{9, q}^{1}$ \\
\hline 10 & $\mathbb{Z} / 3$ & 0 & 0 & 0 & 0 & 0 & 0 & 0 & $(\mathbb{Z} / 3)$ \\
9 & $\mathbb{Z} / 3$ & $(\mathbb{Z} / 3)$ & 0 & 0 & 0 & 0 & 0 & 0 & 0 \\
8 & $\mathbb{Z} / 3$ & 0 & $(\mathbb{Z} / 3)$ & 0 & 0 & 0 & 0 & 0 & 0 \\
7 & 0 & 0 & $(\mathbb{Z} / 3)$ & 0 & 0 & 0 & 0 & 0 & 0 \\
6 & 0 & 0 & 0 & 0 & 0 & 0 & 0 & 0 & 0 \\
5 & 0 & 0 & 0 & 0 & 0 & 0 & 0 & 0 & 0 \\
4 & $(\mathbb{Z} / 3)$ & 0 & 0 & 0 & 0 & 0 & 0 & 0 & 0
\end{tabular}

Table 19: The initial terms in the spectral sequence (11-12) for $n=5$ and $A=\mathbb{Z} / 3$

\section{Appendix A The derived Koszul complex}

In this appendix, we illustrate our derived functor methods, by giving an explicit description of certain objects and morphisms obtained by deriving the Koszul sequence (2-30).

Let

$$
0 \rightarrow L \stackrel{\delta}{\rightarrow} M \rightarrow A \rightarrow 0
$$

be a flat resolution of the abelian group $A$. A convenient model for the derived category object $L \Lambda^{n}(A)$ is provided by the dual Koszul complex of the morphism $\delta: L \rightarrow M$. Recall that for $n=2$ this is the complex

$$
\Gamma_{2}(L) \stackrel{\delta_{2}}{\rightarrow} L \otimes M \stackrel{\delta_{1}}{\rightarrow} \Lambda^{2}(M)
$$

with the differentials

$$
\begin{aligned}
& \delta_{2}\left(\gamma_{2}(l)\right)=l \otimes \delta(l) \\
& \delta_{1}(l \otimes m)=\delta(l) \wedge m
\end{aligned}
$$

and for $n=3$ the complex

$$
\Gamma_{3}(L) \stackrel{\delta_{3}}{\rightarrow} \Gamma_{2}(L) \otimes M \stackrel{\delta_{2}}{\rightarrow} L \otimes \Lambda^{2}(M) \stackrel{\delta_{1}}{\rightarrow} \Lambda^{3}(M)
$$

with the differentials

$$
\begin{aligned}
\delta_{3}\left(\gamma_{3}(l)\right) & =\gamma_{2}(l) \otimes \delta(l) \\
\delta_{3}\left(\gamma_{2}(l) l^{\prime}\right) & =l l^{\prime} \otimes \delta(l)+\gamma_{2}(l) \otimes \delta\left(l^{\prime}\right) \\
\delta_{2}\left(\gamma_{2}(l) \otimes m\right) & =l \otimes m \wedge \delta(l) \\
\delta_{1}\left(l \otimes m \wedge m^{\prime}\right) & =\delta(l) \wedge m \wedge m^{\prime} .
\end{aligned}
$$


The derived category object

$$
L \Lambda^{2}(A) \stackrel{L}{\otimes} A
$$

may be represented by the tensor product of the complex (A-1) with the complex $\delta: L \rightarrow M$, in other words by the total complex associated to the bicomplex

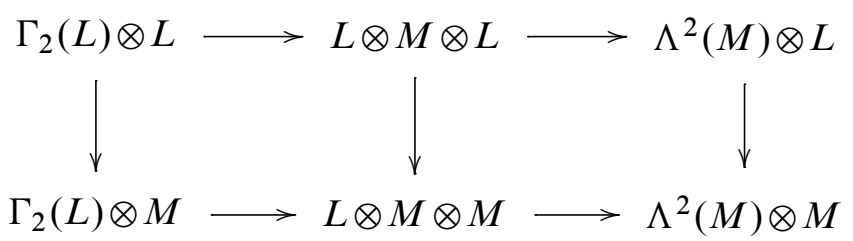

in other words the complex

$(\mathrm{A}-2) \quad \Gamma_{2}(L) \otimes L \stackrel{\delta_{3}^{\prime}}{\rightarrow} \Gamma_{2}(L) \otimes M \oplus(L \otimes M \otimes L)$

$$
\stackrel{\delta_{2}^{\prime}}{\rightarrow}(L \otimes M \otimes M) \oplus \Lambda^{2}(M) \otimes L \stackrel{\delta_{1}^{\prime}}{\rightarrow} \Lambda^{2}(M) \otimes M
$$

with differentials

$$
\begin{aligned}
\delta_{3}^{\prime}\left(\gamma_{2}(l) \otimes l^{\prime}\right) & =\left(\gamma_{2}(l) \otimes \delta\left(l^{\prime}\right),-l \otimes \delta(l) \otimes l^{\prime}\right) \\
\delta_{2}^{\prime}\left(\gamma_{2}(l) \otimes m\right) & =(l \otimes \delta(l) \otimes m, 0) \\
\delta_{2}^{\prime}\left(l \otimes m \otimes l^{\prime}\right) & =\left(l \otimes m \otimes \delta\left(l^{\prime}\right), m \wedge \delta(l) \otimes l^{\prime}\right) \\
\delta_{1}^{\prime}\left(l \otimes m \otimes m^{\prime}\right) & =\delta(l) \wedge m \otimes m^{\prime} \\
\delta_{1}^{\prime}\left(m \wedge m^{\prime} \otimes l\right) & =m \wedge m^{\prime} \otimes \delta(l) .
\end{aligned}
$$

Recall that

$$
\begin{aligned}
L_{1} \Lambda^{2}(A) & =\Omega_{2}(A) \\
\pi_{2}\left(L \Lambda^{2}(A) \stackrel{L}{\otimes} A\right) & =\operatorname{Tor}\left(\Omega_{2}(A), A\right) .
\end{aligned}
$$

Given elements $a, a^{\prime} \in{ }_{n} A$, let us choose its representatives $m, m^{\prime} \in M$ and so-called cross-cap elements $l, l^{\prime} \in L$, for which $\delta(l)=n m$ and $\delta\left(l^{\prime}\right)=n m^{\prime}$. The maps

$$
\begin{gathered}
\Omega_{2}(A) \rightarrow(L \otimes M) / \operatorname{im}\left(\delta_{2}\right) \\
\operatorname{Tor}\left(\Omega_{2}(A), A\right) \rightarrow\left(\Gamma_{2}(L) \otimes M \oplus(L \otimes M \otimes L)\right) / \operatorname{im}\left(\delta_{3}^{\prime}\right)
\end{gathered}
$$

which define the isomorphisms (A-3) and (A-4) are given by

$$
\begin{aligned}
\omega_{2}^{n}(a) & \mapsto l \otimes m+\operatorname{im}\left(\delta_{2}\right) \\
\tau_{n}\left(\omega_{2}^{n}(a), b\right) & \mapsto\left(-\gamma_{2}(l) \otimes m^{\prime}, l \otimes m \otimes l^{\prime}\right)+\operatorname{im}\left(\delta_{3}\right) .
\end{aligned}
$$


Next we consider the diagram with exact rows and columns

$(\mathrm{A}-5)$

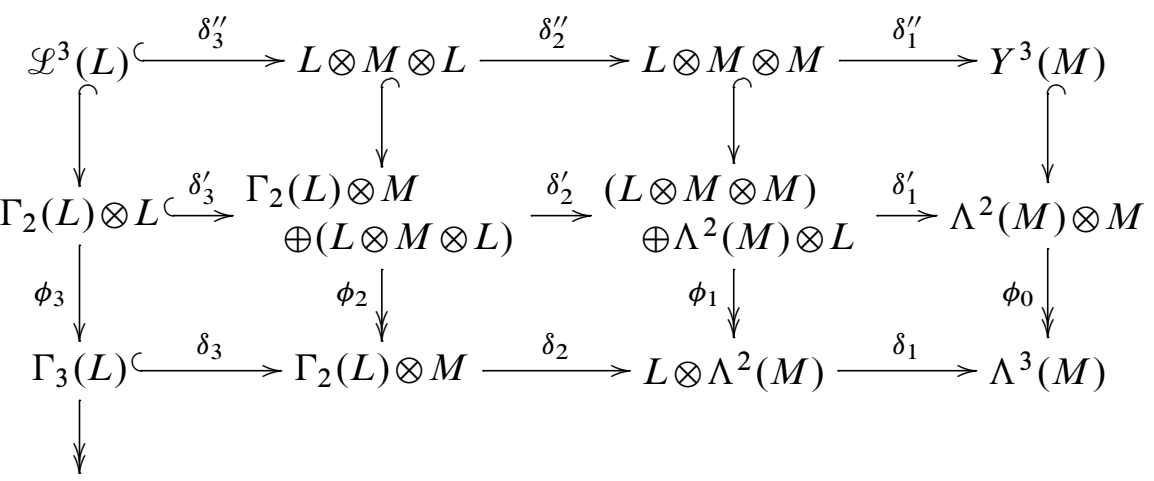

$L \otimes \mathbb{Z} / 3$

with $\quad \phi_{0}\left(m \wedge m^{\prime} \otimes m^{\prime \prime}\right)=m \wedge m^{\prime} \wedge m^{\prime \prime}$

$\phi_{1}\left(l \otimes m \otimes m^{\prime}\right)=l \otimes m \wedge m^{\prime}$

$\phi_{1}\left(m \wedge m^{\prime} \otimes l\right)=l \otimes m \wedge m^{\prime}$

$\phi_{2}\left(\gamma_{2}(l) \otimes m\right)=-\gamma_{2}(l) \otimes m$

$\phi_{2}\left(l \otimes m \otimes l^{\prime}\right)=l l^{\prime} \otimes m$

$\phi_{3}\left(\gamma_{2}(l) \otimes l^{\prime}\right)=-\gamma_{2}(l) l^{\prime}$

and

$\delta_{3}^{\prime \prime}\left(\overline{l \otimes l^{\prime} \wedge l^{\prime \prime}}\right)=l \otimes \delta\left(l^{\prime \prime}\right) \otimes l^{\prime}+l^{\prime \prime} \otimes \delta(l) \otimes l^{\prime}-l \otimes \delta\left(l^{\prime}\right) \otimes l^{\prime \prime}-l^{\prime} \otimes \delta(l) \otimes l^{\prime \prime}$

$\delta_{2}^{\prime \prime}\left(l \otimes m \otimes l^{\prime}\right)=l \otimes \delta\left(l^{\prime}\right) \otimes m+l^{\prime} \otimes \delta(l) \otimes m+l \otimes m \otimes \delta\left(l^{\prime}\right)$

$\delta_{1}^{\prime \prime}\left(l \otimes m \otimes m^{\prime}\right)=\left\{\delta(l), m^{\prime}, m\right\}$.

Here $\overline{l \otimes l^{\prime} \wedge l^{\prime \prime}}$ denotes the image of the element $l \otimes l^{\prime} \wedge l^{\prime \prime}$ under the natural epimorphism $L \otimes \Lambda^{2}(L) \rightarrow \mathscr{L}^{3}(L)$. We will now make use of the fact that the dual de Rham complex

$$
0 \rightarrow \Lambda^{3}(L) \rightarrow L \otimes \Lambda^{2}(L) \rightarrow \Gamma_{2}(L) \otimes L \rightarrow \Gamma_{3}(L)
$$

has trivial homology in positive dimensions and hence

$$
\mathscr{L}^{3}(L)=\operatorname{ker}\left\{\Gamma_{2}(L) \otimes L \rightarrow \Gamma_{3}(L)\right\}=\operatorname{coker}\left\{\Lambda^{3}(L) \rightarrow L \otimes \Lambda^{2}(L)\right\} .
$$

Consider the functor $\bar{E}^{3}(A):=\operatorname{im}\left\{\Gamma_{2}(A) \otimes A \rightarrow \Gamma_{3}(A)\right\}$. We have a natural short exact sequence

$$
0 \rightarrow \bar{E}^{3}(A) \rightarrow \Gamma_{3}(A) \rightarrow A \otimes \mathbb{Z} / 3 \rightarrow 0
$$


which induces the following commutative diagram:

(A-6)

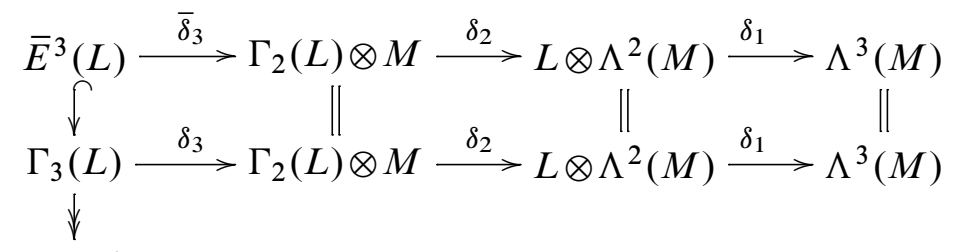

$L \otimes \mathbb{Z} / 3$

From diagrams (A-5) and (A-6) we deduce the following diagram with exact arrows and columns

$(\mathrm{A}-7)$

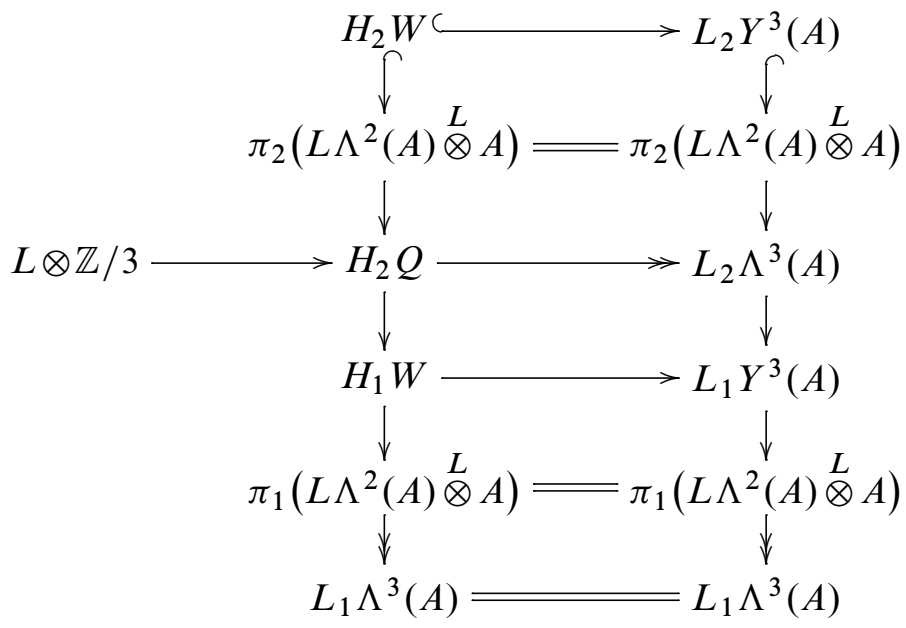

where $W$ and $Q$ are the upper rows in diagrams (A-5) and (A-6) respectively. We give the following simple example, which illustrates the inner life of the previous diagrams.

\section{Proposition A.1}

$$
L_{i} Y^{3}(\mathbb{Z} / 3)= \begin{cases}\mathbb{Z} / 3 & i=2, \\ \mathbb{Z} / 9 & i=1, \\ 0 & i \neq 1,2\end{cases}
$$

Proof It follows from the description $Y^{3}(A)=\operatorname{ker}\left\{\Lambda^{2}(A) \otimes A \rightarrow \Lambda^{3}(A)\right\}$ that

$$
L_{2} Y^{3}(A)=\operatorname{ker}\left\{\operatorname{Tor}\left(\Omega_{2}(A), A\right) \rightarrow L_{2} \Lambda^{3}(A)\right\} .
$$

Let $A=\mathbb{Z} / 3$ and

$$
L \stackrel{\partial}{\rightarrow} M \quad \text { is } \quad \mathbb{Z} \stackrel{3}{\rightarrow} \mathbb{Z} .
$$

Let $l, m$ be generators of $L$ and $M$ resp. with $\delta(l)=3 m$. The group $\operatorname{Tor}\left(\Omega_{2}(A), A\right)$ is generated by the homology class of the element $\left(\gamma_{2}(l) \otimes m, l \otimes m \otimes l\right)$ in the com- 
plex (A-2). We have

$\phi_{2}\left(\gamma_{2}(l) \otimes m, l \otimes m \otimes l\right)=\gamma_{2}(l) \otimes m+l l \otimes m=3 \gamma_{2}(l) \otimes m=\gamma_{2}(l) \otimes \delta(l)=\delta_{3}\left(\gamma_{3}(l)\right)$.

Hence

$$
\operatorname{Tor}\left(\Omega_{2}(A), A\right) \rightarrow L_{2} \Lambda^{3}(A)
$$

induced by the map $\phi_{2}$ is the zero map. This proves that

$$
L_{2} Y^{3}(\mathbb{Z} / 3)=\mathbb{Z} / 3 \text {. }
$$

It is easy to see that for our choice of $L$ and $M$, the complex $W$ has the form

$$
\mathbb{Z} \stackrel{9}{\rightarrow} \mathbb{Z} \rightarrow 0
$$

so that $H_{1} W=\mathbb{Z} / 9, H_{2} W=0$. In this case, the diagram (A-7) has the form

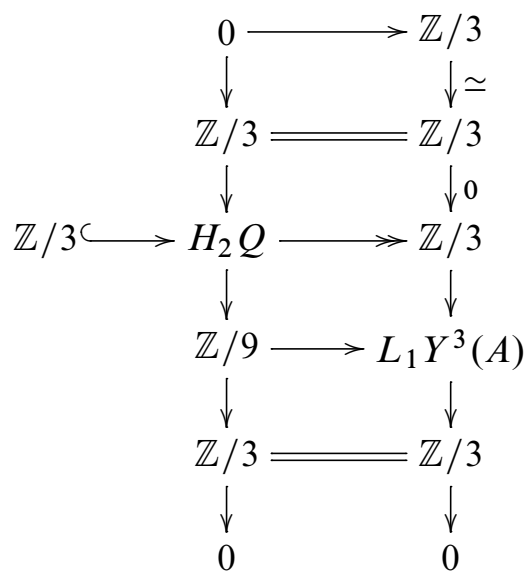

and we see that the map $H_{1} W \rightarrow L_{1} Y^{3}(A)$ is an isomorphism and hence

$$
L_{1} Y^{3}(\mathbb{Z} / 3)=\mathbb{Z} / 9 \text {. }
$$

The object $L \Gamma_{2}(A)$ of the derived category may be represented as the following complex:

$$
L \otimes L \rightarrow \Gamma_{2}(L) \otimes(M \otimes L) \rightarrow \Gamma_{2}(M)
$$

Consider the following diagram:

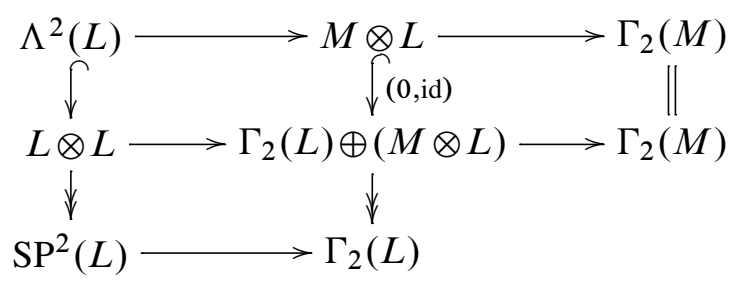


Denote by

$$
C=C(L \stackrel{\delta}{\rightarrow} M)
$$

the upper complex in (A-8). Diagram (A-8) implies the following exact sequence of homology groups:

$$
0 \rightarrow H_{1} C \rightarrow R_{2}(A) \rightarrow L \otimes \mathbb{Z} / 2 \rightarrow H_{0} C \rightarrow \Gamma_{2}(A) \rightarrow 0
$$

In particular, the $p$-torsion components of $H_{i} C$ and $L_{i} \Gamma_{2}$ are naturally isomorphic for $p \neq 2$.

The objects

$$
L \Gamma_{3}(A) \quad \text { and } \quad A \stackrel{L}{\otimes} L \Gamma_{2}(A)
$$

of the derived category may be represented by the following complexes:

(A-9) $\quad L \otimes L \otimes L \rightarrow\left(\Gamma_{2}(L) \otimes L\right) \oplus\left(L \otimes \Gamma_{2}(L)\right) \oplus(L \otimes L \otimes M)$

$$
\rightarrow \Gamma_{3}(L) \oplus\left(\Gamma_{2}(L) \otimes M\right) \oplus\left(L \oplus \Gamma_{2}(M)\right) \rightarrow \Gamma_{3}(M)
$$

(A-10) $L \otimes L \otimes L \rightarrow(M \otimes L \otimes L) \oplus\left(L \otimes \Gamma_{2}(L)\right) \oplus(L \otimes L \otimes M)$

$$
\rightarrow\left(L \otimes \Gamma_{2}(M)\right) \oplus\left(M \otimes \Gamma_{2}(L)\right) \oplus(M \otimes L \otimes M) \rightarrow M \otimes \Gamma_{2}(M)
$$

Consider the following diagram:

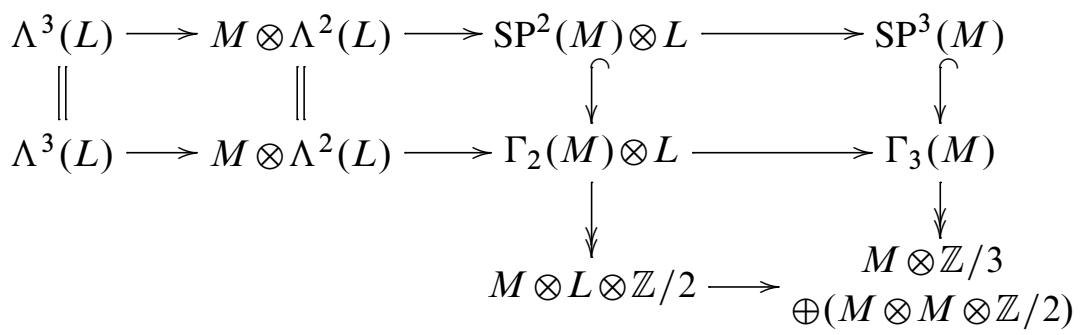

The upper complex in (A-11) is a model for the element $L \mathrm{SP}^{3}(A)$ in the derived category. Denote the middle horizontal complex by

$$
D=D(L \stackrel{\delta}{\rightarrow} M)
$$

We have the natural isomorphism

$$
H_{2} D \simeq L_{2} \operatorname{SP}^{3}(A)
$$

and the following exact sequence:

$(\mathrm{A}-12) \quad 0 \rightarrow L_{1} \mathrm{SP}^{3}(A) \rightarrow H_{1} D \rightarrow \operatorname{Tor}(M \otimes A, \mathbb{Z} / 2)$

$$
\rightarrow \mathrm{SP}^{3}(A) \rightarrow H_{0} D \rightarrow M \otimes \mathbb{Z} / 3 \oplus(M \otimes A \otimes \mathbb{Z} / 2) \rightarrow 0
$$


Now consider the following diagram which extends the diagram (A-5):

(A-13)
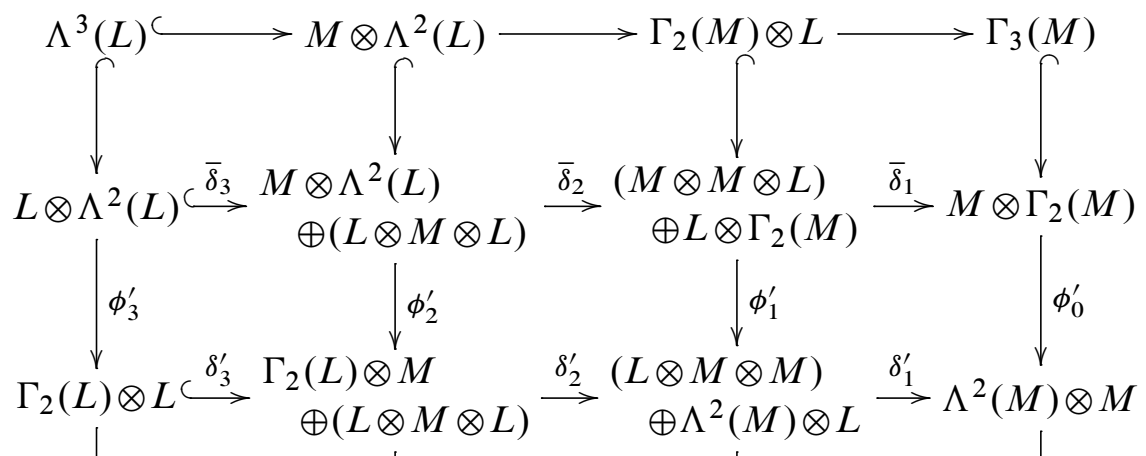

Here $\quad \bar{\delta}_{1}\left(m \otimes m^{\prime} \otimes l\right)=m \otimes m^{\prime} \delta(l)$

$$
\begin{aligned}
& \bar{\delta}_{1}\left(l \otimes \gamma_{2}(m)\right)=\delta(l) \otimes \gamma_{2}(m) \\
& \bar{\delta}_{2}\left(m \otimes l \wedge l^{\prime}\right)=\left(m \otimes \delta(l) \otimes l^{\prime}-m \otimes \delta\left(l^{\prime}\right) \otimes l, 0\right) \\
& \bar{\delta}_{2}\left(l \otimes m \otimes l^{\prime}\right)=\left(\delta(l) \otimes m \otimes l^{\prime},-l \otimes m \delta(l)\right) \\
& \bar{\delta}_{3}\left(l \otimes l^{\prime} \wedge l^{\prime \prime}\right)=\left(\delta(l) \otimes l^{\prime} \wedge l^{\prime \prime},-l \otimes \delta\left(l^{\prime}\right) \otimes l^{\prime \prime}+l \otimes \delta\left(l^{\prime \prime}\right) \otimes l^{\prime}\right)
\end{aligned}
$$

and

$$
\begin{aligned}
\phi_{0}^{\prime}\left(m \otimes \gamma_{2}\left(m^{\prime}\right)\right) & =m \wedge m^{\prime} \otimes m^{\prime} \\
\phi_{1}^{\prime}\left(m \otimes m^{\prime} \otimes l\right) & =\left(-l \otimes m \otimes m^{\prime}, m \wedge m^{\prime} \otimes l\right) \\
\phi_{1}^{\prime}\left(l \otimes \gamma_{2}(m)\right) & =(l \otimes m \otimes m, 0) \\
\phi_{2}^{\prime}\left(l \otimes m \otimes l^{\prime}\right) & =\left(-l l^{\prime} \otimes m,-l \otimes m \otimes l^{\prime}\right) \\
\phi_{2}^{\prime}\left(m \otimes l \wedge l^{\prime}\right) & =\left(0, l \otimes m \otimes l^{\prime}-l^{\prime} \otimes m \otimes l\right) \\
\phi_{3}^{\prime}\left(l \otimes l^{\prime} \wedge l^{\prime \prime}\right) & =-l l^{\prime} \otimes l^{\prime \prime}+l l^{\prime \prime} \otimes l^{\prime} .
\end{aligned}
$$

We obtain the natural isomorphism of complexes:

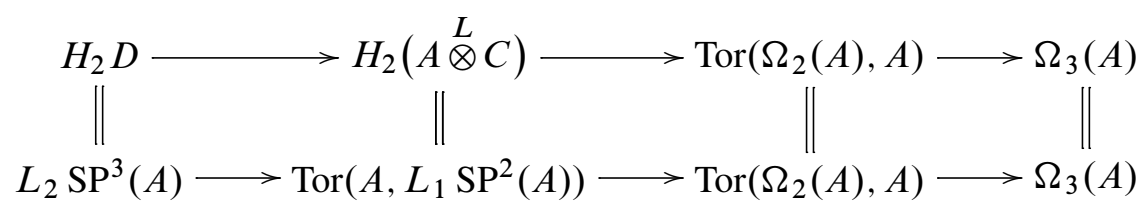


The map

$$
\operatorname{Tor}\left(A, L_{1} \mathrm{SP}^{2}(A)\right) \rightarrow\left(M \otimes \Lambda^{2}(L) \oplus(L \otimes M \otimes L)\right) / \operatorname{im}\left(\bar{\delta}_{3}\right)
$$

is given as follows: let $a, a^{\prime}, a^{\prime \prime} \in A$ with $n a=n a^{\prime}=n a^{\prime \prime}=0$ are represented by elements $m, m^{\prime}, m^{\prime \prime}$ is $M$ and $\delta(l)=n m, \delta\left(l^{\prime}\right)=n m^{\prime}, \delta\left(l^{\prime \prime}\right)=n m^{\prime \prime}$, then

$$
\tau_{n}\left(a, \beta_{n}\left(a^{\prime}, a^{\prime \prime}\right)\right) \mapsto\left(-m \otimes l^{\prime} \wedge l^{\prime \prime}, l \otimes m^{\prime} \otimes l^{\prime \prime}-l \otimes m^{\prime \prime} \otimes l^{\prime}\right)+\operatorname{im}\left(\bar{\delta}_{3}\right) .
$$

The map $\phi_{2}^{\prime}$ induces the Koszul-type map

$$
\operatorname{Tor}\left(A, L_{1} \mathrm{SP}^{2}(A)\right) \rightarrow \operatorname{Tor}\left(\Omega_{2}(A), A\right)
$$

defined by

$$
\begin{aligned}
& \tau_{n}\left(a, \beta_{n}\left(a^{\prime}, a^{\prime \prime}\right)\right) \\
& \quad \mapsto\left(\tau_{n}\left(\omega_{2}^{n}\left(a+a^{\prime \prime}\right)-\omega_{2}^{n}(a)-\omega_{2}^{n}\left(a^{\prime \prime}\right), a^{\prime}\right)-\left(\tau_{n}\left(\omega_{2}^{n}\left(a+a^{\prime}\right)-\omega_{2}^{n}(a)-\omega_{2}^{n}\left(a^{\prime}\right), a^{\prime \prime}\right) .\right.\right.
\end{aligned}
$$

Example In the case

$$
(L \stackrel{\delta}{\rightarrow} M)=(\mathbb{Z} \stackrel{n}{\rightarrow} \mathbb{Z}),
$$

the diagram (A-13) has the following form:

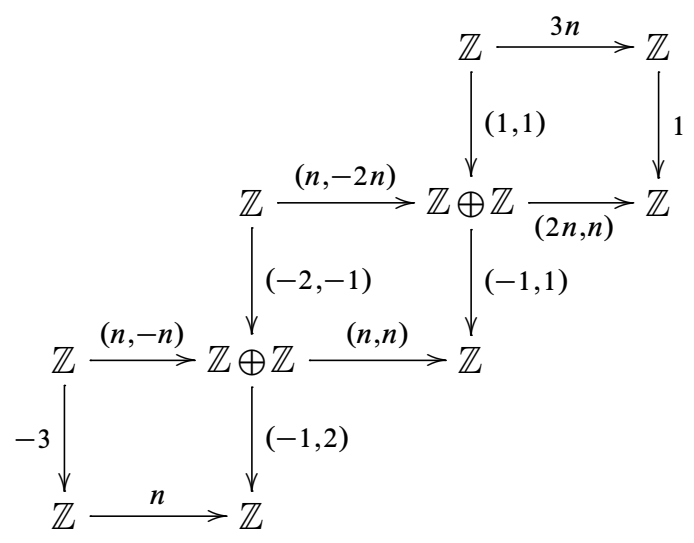

This diagram implies that the map

$$
H_{1}(\mathbb{Z} / n \stackrel{L}{\otimes} C(\mathbb{Z} \stackrel{n}{\rightarrow} \mathbb{Z})) \rightarrow \pi_{1}\left(L \Lambda^{2}(\mathbb{Z} / n) \stackrel{L}{\otimes} \mathbb{Z} / n\right)
$$

is multiplication by 3 in the group $\mathbb{Z} / n$.

When $A=\mathbb{Z} / 2$ and

$$
C=C(\mathbb{Z} \stackrel{2}{\rightarrow} \mathbb{Z}),
$$


we have the following commutative diagram:

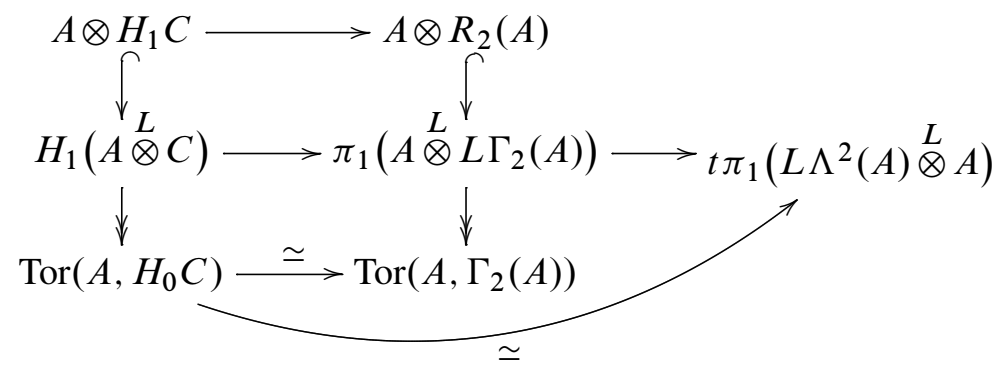

As a corollary, we find that:

Proposition A.2 The derived Koszul map

$$
\pi_{1}\left(A \stackrel{L}{\otimes} L \Gamma_{2}(A)\right) \rightarrow \pi_{1}\left(L \Lambda^{2}(A) \stackrel{L}{\otimes} A\right)
$$

is the zero map for $A=\mathbb{Z} / 3$ and an epimorphism for $A=\mathbb{Z} / p$ whenever $p$ is a prime $\neq 3$.

\section{References}

[1] K Akin, D A Buchsbaum, J Weyman, Schur functors and Schur complexes, Adv. in Math. 44 (1982) 207-278 MR658729

[2] H-J Baues, Homotopy type and homology, Oxford Math. Monogr., Oxford Science Publ., The Clarendon Press, Oxford Univ. Press, New York (1996) MR1404516

[3] H J Baues, J Buth, On the group of homotopy equivalences of simply connected five manifolds, Math. Z. 222 (1996) 573-614 MR1406269

[4] H-J Baues, P Goerss, A homotopy operation spectral sequence for the computation of homotopy groups, Topology 39 (2000) 161-192 MR1710998

[5] H-J Baues, T Pirashvili, A universal coefficient theorem for quadratic functors, J. Pure Appl. Algebra 148 (2000) 1-15 MR1750731

[6] D Blanc, C Stover, A generalized Grothendieck spectral sequence, from: "Adams Memorial Symposium on Algebraic Topology, 1 (Manchester, 1990)”, (N Ray, G Walker, editors), London Math. Soc. Lecture Note Ser. 175, Cambridge Univ. Press (1992) 145-161 MR1170576

[7] A K Bousfield, Homogeneous functors and their derived functors, Unpublished manuscript, Brandeis University (1967)

[8] A K Bousfield, Operations on derived functors of nonadditive functors, Unpublished manuscript, Brandeis University (1967) 
[9] A K Bousfield, E B Curtis, D M Kan, D G Quillen, D L Rector, J W Schlesinger, The mod-p lower central series and the Adams spectral sequence, Topology 5 (1966) 331-342 MR0199862

[10] L Breen, On the functorial homology of abelian groups, J. Pure Appl. Algebra 142 (1999) 199-237 MR1721092

[11] H Cartan, J C Moore, R Thom, J-P Serre, Algèbres d'Eilenberg-Mac Lane et homotopie, Séminaire Henri Cartan de l'Ecole Normale Supérieure 1954/1955, Secrétariat math., Paris (1955) MR0087934

[12] F R Cohen, J C Moore, J A Neisendorfer, Torsion in homotopy groups, Ann. of Math. (2) 109 (1979) 121-168 MR519355

[13] F R Cohen, J Wu, A remark on the homotopy groups of $\Sigma^{n} \mathbf{R} \mathrm{P}^{2}$, from: "The Čech centennial (Boston, MA, 1993)”, (M Cenkl, H Miller, editors), Contemp. Math. 181, Amer. Math. Soc. (1995) 65-81 MR1320988

[14] E B Curtis, Lower central series of semi-simplicial complexes, Topology 2 (1963) 159-171 MR0156343

[15] E B Curtis, Some relations between homotopy and homology, Ann. of Math. (2) 82 (1965) 386-413 MR0184231

[16] E B Curtis, Simplicial homotopy theory, Advances in Math. 6 (1971) 107-209 MR0279808

[17] G J Decker, The integral homology algebra of an Eilenberg-Mac Lane space, PhD thesis, University of Chicago (1974) Available at www.maths.abdn.ac.uk/ bensondj/html/archive/decker.html

[18] A Dold, Homology of symmetric products and other functors of complexes, Ann. of Math. (2) 68 (1958) 54-80 MR0097057

[19] A Dold, Lectures on algebraic topology, second edition, Grund. der Math. Wissenschaften 200, Springer, Berlin (1980) MR606196

[20] A Dold, D Puppe, Homologie nicht-additiver Funktoren. Anwendungen, Ann. Inst. Fourier Grenoble 11 (1961) 201-312 MR0150183

[21] W Dreckmann, Distributivgesetze in der Homotopietheorie, PhD Thesis, Rheinische Friedrich-Wilhelms-Universität Bonn (1992); Bonner Math. Schriften 249 (1993) MR1286734

[22] S Eilenberg, S Mac Lane, On the groups $H(\Pi, n)$. II. Methods of computation, Ann. of Math. (2) 60 (1954) 49-139 MR0065162

[23] V Franjou, Cohomologie de de Rham entière arXiv:math/0404123

[24] V Franjou, J Lannes, L Schwartz, Autour de la cohomologie de Mac Lane des corps finis, Invent. Math. 115 (1994) 513-538 MR1262942 
[25] W Fulton, Young tableaux. With applications to representation theory and geometry, London Math. Society Student Texts 35, Cambridge Univ. Press (1997) MR1464693

[26] W Fulton, J Harris, Representation theory. A first course, Graduate Texts in Math. 129, Springer, New York (1991) MR1153249

[27] P Gabriel, M Zisman, Calculus of fractions and homotopy theory, Ergebnisse der Math. und ihrer Grenzgebiete 35, Springer, New York (1967) MR0210125

[28] R M Hamsher, Eilenberg-Mac Lane algebras and their computation. An invariant description of $H(\Pi, 1), \mathrm{PhD}$ thesis, University of Chicago (1973) Available at www.maths.abdn.ac.uk/ bensondj/html/archive/hamsher.html

[29] L Illusie, Complexe cotangent et déformations. I, Lecture Notes in Math. 239, Springer, Berlin (1971) MR0491680

[30] F Jean, Foncteurs dérivés de l'algébre symétrique: Application au calcul de certains groupes d'homologie fonctorielle des espaces $K(B, n), \mathrm{PhD}$ thesis, University of Paris 13 (2002) Available at www.maths.abdn.ac.uk/ bensondj/html/archive/ jean.html

[31] B Köck, Computing the homology of Koszul complexes, Trans. Amer. Math. Soc. 353 (2001) 3115-3147 MR1828601

[32] A Lascoux, Syzygies des variétés déterminantales, Adv. in Math. 30 (1978) 202-237 MR520233

[33] D Leibowitz, The $E^{1}$ term of the lower central series spectral sequence for the homotopy of spaces, $\mathrm{PhD}$ thesis, Brandeis University (1972)

[34] S Mac Lane, Triple torsion products and multiple Künneth formulas, Math. Ann. 140 (1960) 51-64 MR0114843

[35] S Mac Lane, Decker's sharper Künneth formula, from: “Categorical algebra and its applications (Louvain-La-Neuve, 1987)”, (F Borceux, editor), Lecture Notes in Math. 1348, Springer, Berlin (1988) 242-256 MR975974

[36] W Magnus, Über Beziehungen zwischen höheren Kommutatoren., J. Reine Angew. Math. 177 (1937) 105-115

[37] J P May, Simplicial objects in algebraic topology, Van Nostrand Math. Studies 11, Van Nostrand, Princeton-Toronto-London (1967) MR0222892

[38] R Mikhailov, Homotopy theory of Lie functors, in preparation

[39] R Mikhailov, On the homology of the dual de Rham complex arXiv:1001.2824

[40] R Mikhailov, I B S Passi, Lower central and dimension series of groups, Lecture Notes in Math. 1952, Springer, Berlin (2009) MR2460089

[41] R Mikhailov, J Wu, On homotopy groups of the suspended classifying spaces, Algebr. Geom. Topol. 10 (2010) 565-625 MR2602844 
[42] J A Neisendorfer, 3-primary exponents, Math. Proc. Cambridge Philos. Soc. 90 (1981) 63-83 MR611286

[43] D Quillen, On the (co-) homology of commutative rings, from: "Applications of categorical algebra”, (A Heller, editor), Proc. Sympos. Pure Math. 17, (New York, 1968) Amer. Math. Soc. (1970) 65-87 MR0257068

[44] N Roby, Lois polynomes et lois formelles en théorie des modules, Ann. Sci. École Norm. Sup. (3) 80 (1963) 213-348 MR0161887

[45] J W Schlesinger, The semi-simplicial free Lie ring, Trans. Amer. Math. Soc. 122 (1966) 436-442 MR0199861

[46] H Toda, Composition methods in homotopy groups of spheres, Annals of Math. Studies 49, Princeton Univ. Press (1962) MR0143217

[47] C A Weibel, An introduction to homological algebra, Cambridge Studies in Advanced Math. 38, Cambridge Univ. Press (1994) MR1269324

[48] J H C Whitehead, A certain exact sequence, Ann. of Math. (2) 52 (1950) 51-110 MR0035997

[49] E Witt, Treue Darstellung Liescher Ringe, J. Reine Angew. Math. 177 (1937) 152-160

[50] $\mathbf{J} \mathbf{W u}$, Homotopy theory of the suspensions of the projective plane, Mem. Amer. Math. Soc. 162 no. 769, Amer. Math. Soc. (2003) MR1955357

Laboratoire CNRS LAGA, Universite Paris 13

99, avenue Jean-Baptiste Clement, 93430 Villetaneuse, France

Department of Algebra, Steklov Mathematical Institute

Gubkina 8, Moscow, 119991, Russia

breen@math.univ-paris13.fr, romanvm@mi.ras.ru

http://www.math.univ-paris13.fr/ breen, http://www.mi.ras.ru/ romanvm/

Received: 18 January 2010 